\title{
WestVirginiaUniversity
}

THE RESEARCH REPOSITORY @ WVU

Graduate Theses, Dissertations, and Problem Reports

2000

\section{Effects of aging on bond between FRP and concrete}

John Dyer Barger

West Virginia University

Follow this and additional works at: https://researchrepository.wvu.edu/etd

\section{Recommended Citation}

Barger, John Dyer, "Effects of aging on bond between FRP and concrete" (2000). Graduate Theses,

Dissertations, and Problem Reports. 1011.

https://researchrepository.wvu.edu/etd/1011

This Thesis is protected by copyright and/or related rights. It has been brought to you by the The Research Repository @ WVU with permission from the rights-holder(s). You are free to use this Thesis in any way that is permitted by the copyright and related rights legislation that applies to your use. For other uses you must obtain permission from the rights-holder(s) directly, unless additional rights are indicated by a Creative Commons license in the record and/ or on the work itself. This Thesis has been accepted for inclusion in WVU Graduate Theses, Dissertations, and Problem Reports collection by an authorized administrator of The Research Repository @ WVU. For more information, please contact researchrepository@mail.wvu.edu. 


\title{
Effects of Aging on Bond Between FRP and Concrete
}

\author{
John D. Barger
}

Thesis

Submitted to the College of Engineering and Mineral Resources

at

West Virginia University

In Partial Fulfillment of the Requirements for

The Degree of Master of Science

in

Civil Engineering

\author{
Hota GangaRao, Ph.D., Chair \\ Rakesh Gupta, Ph.D. \\ P.V. Vijay, Ph.D. \\ Department of Civil Engineering
}

Morgantown,West Virginia

2000

Keywords: Fiber Reinforced Polymers, Adhesive, Bond, Carbon Fiber, Glass Fiber

Copyright 2000 John D. Barger 


\title{
Abstract \\ Effects of Aging on the Bond Between FRP and Concrete
}

\author{
John D. Barger
}

Fiber Reinforced Polymer (FRP) composites are continuing to gain prominence for structural and non-structural applications all across the world. FRP composites are being used as complete structural systems or in conjunction with conventional materials leading to hybrid structures. The study of using FRP rebars and wraps to reinforce our nation's existing bridge decks, beams and columns is proving that they are economical as well as future material of choice.

This research deals with the bond behavior of FRP composite wraps adhered/bonded to concrete surface. Focus of the study is on the bond degradation when the hybrid structure is exposed to different adverse environmental conditions including sustained stress. The study involves using an epoxy based system to bond glass and carbon fabrics to concrete. Specimens were aged by means of soaking them in alkaline, water, or acid solutions at either constant temperature or varying temperature for different time durations. The duration of aging ranged from 1 to 9 months. Some of the specimens were conditioned under an applied sustained load. Upon removing the specimens from their conditioning environment, tension tests were performed in the Baldwin machine using specially manufactured concrete cube holder and grip assembly. The specimens were tested before and after aging to determine if any bond degradation occurred under hygrothermal and other chemical conditions. The strain distribution along the bond line was examined. The techniques used in this study for bonding FRP composites to concrete and different test methods including field application of FRP fabrics are also discussed. 


\section{Acknowledgements}

The author wishes to express his sincere appreciation to his academic and research advisor, Dr. Hota GangaRao, for his guidance and knowledge in helping to develop this thesis. Dr. Hota was always available when I needed him and he was extremely helpful when hard times presented themselves. The author would also like to thank Dr. P.V.Vijay for helping me to develop the skills needed in the lab and providing advice and guidance in conducting my research. Both Dr. GangaRao and Dr. Vijay unveiled expertise and knowledge that was astounding. They were always willing to help me and without time restrictions. My experiences with them have bettered my understanding in many areas of engineering. My deepest appreciation goes out to them.

I would also like to thank Dana Humberson and Paul Frum for their help in the lab. Dana has an innovative mind when it comes to designing devices needed to conduct the proper experiments. I thank Dave Turner for his help in teaching me how to use the Baldwin machine for testing.

I also benefited from the assistance given by Steve Rusnock, Jon Killion, and David Boyajian. Without their help the amount of time to perform certain tasks would have been doubled.

I am also thankful for the secretaries Sharon Santos, Sharon Chadderton, and Eleanor Nevera. These women always had a smile on their face and were always willing to assist no matter what.

Financial support for this research was provided by the WVDOT and USDOTFHWA. Their support is greatly appreciated.

Finally, I would like to thank my wife for understanding that things take longer than we sometimes would like them to. The love and appreciation from her as well as my family is invaluable. 


\section{Table Of Contents}

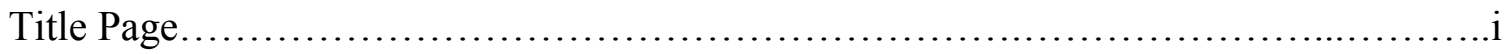

Abstract.......................................................................

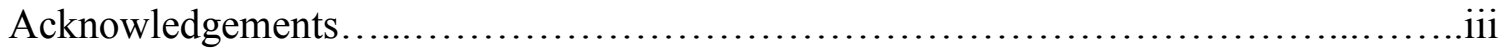

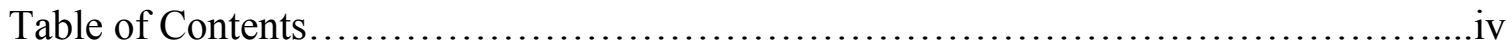

List of Tables.......................................................... vii

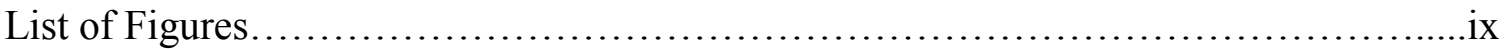

\section{Chapter 1 Introduction}

1.1 General Remarks.................................................1

1.2 Objectives....................................................2

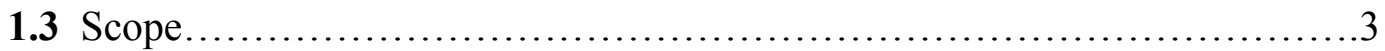

\section{Chapter 2 Literature Review}

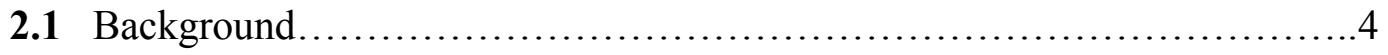

2.2 Review of Aging...................................................

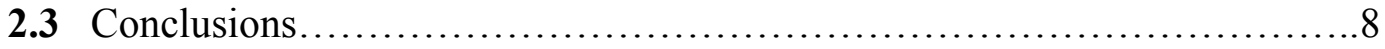

Chapter 3 Materials

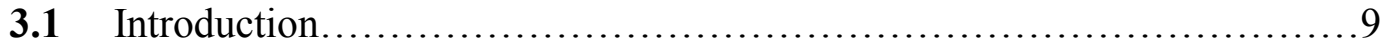

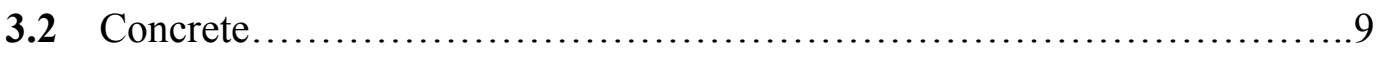

3.3 Carbon and Glass Fibers............................................. 10

3.4 Primer and Adhesive............................................ 12 


\section{Chapter 4 Test Specimens and Experimental Set-Up}

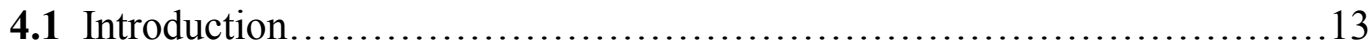

4.2 Specimen Description and Preparation....................................13

4.2.1 Attachment of Strip ......................................... 14

4.3 Instrumentation................................................................

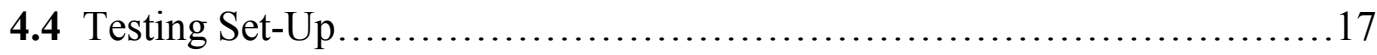

4.5 Test Parameters/Conditioning........................................21

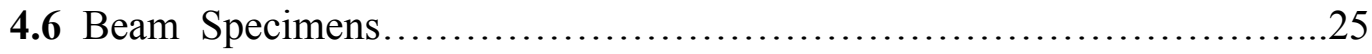

\section{Chapter 5 Experimental Results and Discussion}

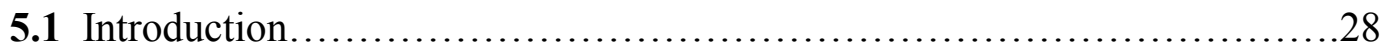

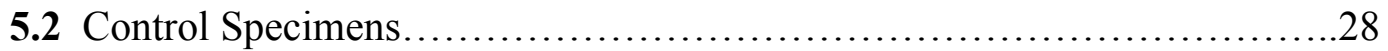

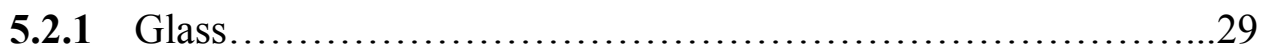

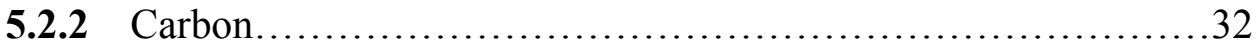

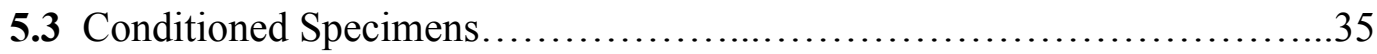

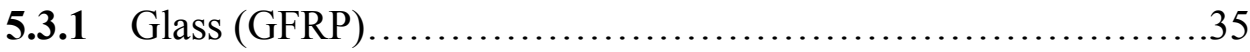

5.3.1.1 Acid and Alkaline—-Room Temperature.................35

5.3.1.2 Acid and Alkaline-Freeze-thaw(FT)..................39

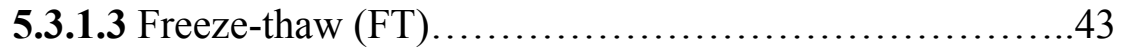

5.3.1.4 Water conditioning .................................47

5.3.1.5 Natural Aging.......................................50

5.3.2 Carbon (CFRP) ...........................................52

5.3.2.1 Acid and Alkaline—Room Temperature ................52

5.3.2.2 Acid and Alkaline-Freeze-thaw(FT) .................56 


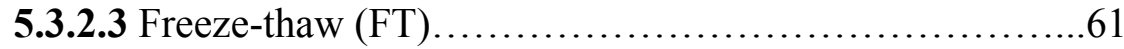

5.3.2.4 Water conditioning .................................65

5.3.2.5 Natural Aging........................................68

5.3.3 Beam Specimens......................................... 70

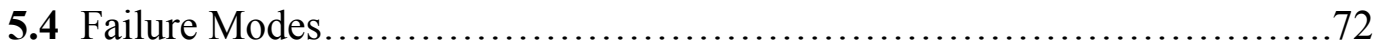

5.5 Discussion of Methods used which may affect results.......................76

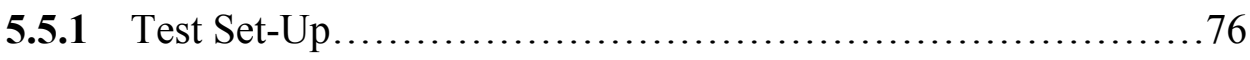

5.5.2 FRP Application............................................77

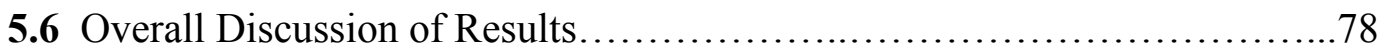

5.7 Correlation of Natural to Accelerated Aging ............................... 81

\section{Chapter 6 Discussion and Conclusions}

6.1 General Remarks and Summary ...................................... 82

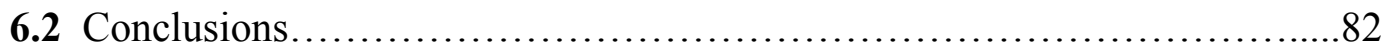

6.3 Recommendations.................................................... 85

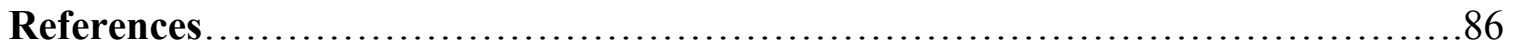

Appendix A

Specifications of Mbrace System.......................................... 88 


\section{List of Tables}

Table $3.1 \quad$ Compressive Strength of Concrete Cylinders.......................... 10

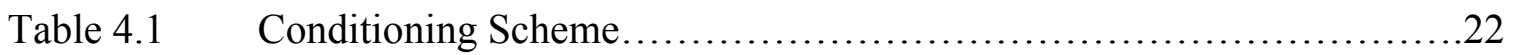

Table $4.2 \quad$ Cycle of Environmental Chamber....................................24

Table $5.1 \quad$ GFRP/Control Stress and Strain Ratios................................31

Table 5.2 CFRP/Control Stress and Strain Ratios..................................34

Table $5.3 \quad$ GFRP/pH 3/RT Stress and Strain Ratios..............................38

Table 5.4 GFRP/pH 13/RT Stress and Strain Ratios..............................38

Table 5.5 GFRP/pH 3/FT Stress and Strain Ratios............................42

Table 5.6 GFRP/pH 13/FT Stress and Strain Ratios..........................42

Table $5.7 \quad$ GFRP/FT Stress and Strain Ratios.................................46

Table $5.8 \quad$ GFRP/FT Stress and Strain Ratios.................................46

Table $5.9 \quad$ GFRP/Water Stress and Strain Ratios..................................49

Table 5.10 GFRP/Natural Aging Stress and Strain Ratios........................51

Table 5.11 CFRP/pH 13/RT Stress and Strain Ratios...........................55

Table 5.12 CFRP/pH 3/RT Stress and Strain Ratios..............................55

Table 5.13 CFRP/pH 13/FT Stress and Strain Ratios............................60

Table 5.14 CFRP/pH 13/FT Stress and Strain Ratios............................60

Table $5.15 \quad$ CFRP/pH 3/FT Stress and Strain Ratios..............................60

Table 5.16 CFRP/FT Stress and Strain Ratios....................................64

Table $5.17 \quad$ CFRP/FT Stress and Strain Ratios...................................64

Table $5.18 \quad$ CFRP/Water Stress and Strain Ratios.................................67 
Table 5.19 CFRP/Naturally Aged Stress and Strain Ratios.......................69

Table 5.20 Stress and Strain Ratios of CFRP/FT for Beam Specimens..............71 


\section{List of Figures}

Figure 3.1 GFRP/CFRP Fabric ......................................... 11

Figure 4.1 Form work for concrete cubes...................................... 14

Figure 4.2 Typical dimensions of specimens.................................. 16

Figure 4.3 Application of pressure when attaching strips.........................16

Figure 4.4 Strain gage detail.............................................. 17

Figure 4.5 Baldwin machine......................................................

Figure 4.6 Grip detail.................................................... 19

Figure 4.7 Application of grips to specimen................................ 19

Figure 4.8 Specimen loaded in baldwin machine.................................20

Figure 4.9 Application of sustained load.....................................21

Figure 4.10a Environmental Chamber.........................................23

Figure $4.10 \mathrm{~b}$ Specimens aging inside chamber................................. 23

Figure 4.11 Natural aging of specimens......................................24

Figure 4.12 Dimensions of beam specimen...................................

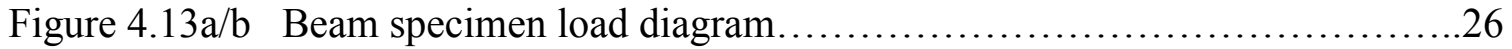

Figure 4.14 Test Set-Up for Beam Specimens.................................27

Figure 5.1 Strain distribution of GFRP control specimen........................29

Figure 5.1a Bond Strength for GFRP control specimens.........................29

Figure 5.2 Strain as a function of location for GFRP control....................... 30

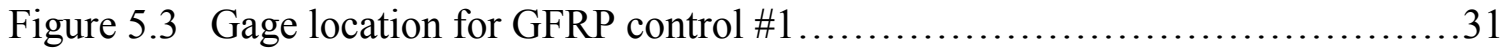

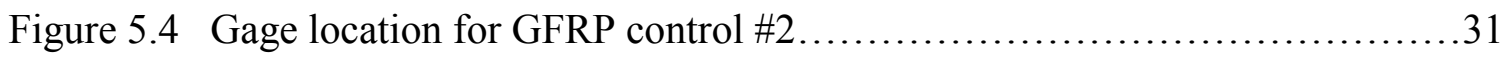

Figure 5.5 Strain distribution of CFRP control specimens..........................32 
Figure 5.6 Strain distribution of CFRP control specimens.........................33

Figure 5.7 Strain as a function of location for CFRP.............................. 33

Figure 5.8 Bond Strength for GFRP in acid and alkaline at room temperature...........35

Figure 5.9 Strain distribution of GFRP in acid at room temperature...................36

Figure 5.10 Strain distribution of GFRP in alkaline at room temperature................37

Figure 5.11 Bond Strength for GFRP in acid and alkaline in chamber.................39

Figure 5.12 Strain distribution of GFRP in acid in chamber........................40

Figure 5.13 Strain distribution of GFRP in alkaline in chamber.....................41

Figure 5.14 Bond Strength for GFRP in chamber................................43

Figure 5.15 Strain distribution of GFRP in chamber............................44

Figure 5.16 Strain distribution of GFRP in chamber...............................45

Figure 5.17 Bond Strength for GFRP in water................................47

Figure 5.18 Strain distribution of GFRP in water...............................48

Figure 5.19 Bond Strength for natural aging of GFRP...........................50

Figure 5.20 Strain distribution of GFRP: natural aging $\ldots \ldots \ldots \ldots \ldots \ldots \ldots \ldots \ldots \ldots \ldots \ldots$

Figure 5.21 Bond Strength for CFRP in acid and alkaline at room temperature.........52

Figure 5.22 Strain distribution of CFRP in acid at room temperature...................53

Figure 5.23 Strain distribution of CFRP in alkaline at room temperature...............54

Figure 5.24 Bond Strength for CFRP in acid and alkaline in chamber.................56

Figure 5.25 Strain distribution of CFRP in acid in chamber.......................57

Figure 5.26 Strain distribution of CFRP in alkaline in chamber.......................58

Figure 5.27 Strain distribution of CFRP in alkaline in chamber....................59

Figure 5.28 Bond Strength for CFRP in chamber................................61 
Figure 5.29 Strain distribution of CFRP in chamber............................62

Figure 5.30 Strain distribution of CFRP in chamber.............................63

Figure 5.31 Bond Strength for CFRP in water................................65

Figure 5.32 Strain distribution of CFRP in water...............................66

Figure 5.33 Bond Strength for Natural Aging of CFRP...........................68

Figure 5.34 Strain distribution of CFRP: natural aging...........................69

Figure 5.35 Bond Strength for beam specimen in chamber......................... 70

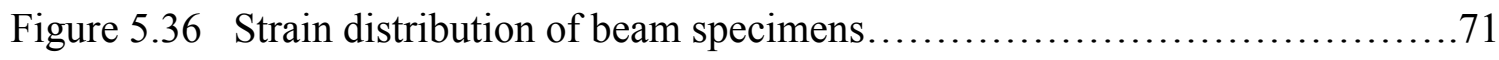

Figure 5.37a/b Adhesive Shear Stresses/Peel Stresses..............................73

Figure 5.38 Failure details.............................................. 74

Figure 5.39 Failure details...................................................... 


\section{Chapter 1}

\section{Introduction}

\subsection{General Remarks}

Corrosion of steel in reinforced concrete is one of the many causes of infrastructure deterioration. This is seen greatly in colder regions of the world where deicing materials are used to keep highways and bridges free of ice and snow. The deicing chemicals seep through micro cracks in concrete and corrode the internal steel reinforcement, which expands and creates tensile stresses in concrete for further deterioration [8]. Therefore, much effort is being put forth to minimize corrosion related degradation. One of the ways to minimize corrosion related deterioration is to use fiber reinforced polymer (FRP) composite rebars in concrete, because of their inertness to electro-chemical reaction.

Over the past 15 years researchers have been studying the effects of fiber reinforced polymer (FRP) rebars in concrete instead of steel to reinforce our nation's bridge decks. Fiber reinforced polymer rebar, as concrete reinforcement, is a promising alternative to steel. However, methods to rehabilitate existing structures that are deteriorating due to the steel corrosion need to be developed. The option of replacing these structures may not be the most economically feasible one due to different reasons such as: high expense involved in development of new land for new construction, loss of productivity associated with traffic and construction delays and adverse impact on local 
businesses and industries [9]. Therefore, research is being carried out at the Constructed Facilities Center (CFC), West Virginia University, to investigate the use of FRP wrapping materials to repair/rehabilitate damaged concrete members including strengthening of existing structural members [5].

The great promise that FRPs demonstrate has elicited a widespread interest and evoked numerous questions regarding the long-term performance of FRPs in service. Innovative attempts have been made to reduce corrosion of steel in concrete, but most have met with limited success. To fully demonstrate the capabilities of FRP fabrics wrapped around steel reinforced concrete members, long term durability, strength and stiffness performance studies are needed. Some work has been carried out with wraps to prove the strength and stiffness enhancements on an economical basis $[6,15,17]$.

\subsection{Objectives}

For a concrete member wrapped with FRP, a high degree of bond between FRP and concrete is essential under short and long-term service conditions. This research focuses on evaluating the long-term bond strength of glass/carbon fabrics with concrete. Degradation of bond strength and mechanical properties of FRP due to aging limits the effectiveness of wrapped structures in terms of strength and serviceability. Understanding the bond behavior under harsh environmental conditions and the results of this study will give designers the knowledge needed to predict the service life; thus providing a safer design. Therefore, the objectives of this study are to:

- Evaluate possible bond strength degradation and the rate of degradation due to alkaline, acid, water, and freeze-thaw conditioning. 
- Evaluate changes in strain distribution along the bond length due to alkaline, acid, water and freeze-thaw conditioning.

- Evaluate parameters influencing bond strength in terms of specimen preparation and test methods.

\subsection{Scope}

More than 100 coupons were tested for bond strength evaluation between concrete and glass or carbon fabric. Aging of the specimens was done through immersion in acidic and alkaline solutions as well as exposure to temperature and humidity changes (refer to Table 4.1, page 22). Specimens were aged with or without sustained stress. Conditioning duration varied between 1 to 9 months. At designated time intervals, the specimens were removed from the conditioning environment and tested. Strain gages were used to evaluate the rate of degradation as well as the strain distribution. A comparison with natural aging and control specimens was made and conclusions were drawn. 


\section{Chapter 2}

\section{Literature Review}

\subsection{Background}

It is apparent that fiber reinforced polymer composites are becoming useful for constructing the world's infrastructure and to rehabilitate and strengthen our existing structures. The utilization of FRP is quickly gaining popularity because of its higher strength to weight ratio as well as its corrosion resistance. The process of externally wrapping concrete beams and columns to strengthen or rehabilitate structures is an area that is receiving much attention. The two major materials, concrete and FRP, are both durable by themselves; but the durability issues are not well understood when the two are chemically bonded. Such lack of understanding may be attributed to bond forces generated from primers and adhesives that are typically used to bond FRP fabrics to concrete. In addition, the application procedures that are necessary to bond the composite to the concrete face need better understanding. Specifically, the bond resistance with time and under harsh environmental conditions needs to be studied to understand the effect of FRP wrap when exposed to different environmental conditions.

\subsection{Review of Aging}

Kshirsagar [12] recently completed a study at West Virginia University that dealt with accelerated environmental aging of FRP-wrapped concrete columns. In his study, 
the influence of six different accelerated aging conditions on the durability of concrete cylinders wrapped with a single layer of a glass fabric embedded in an epoxy resin matrix was examined. Aged specimens were tested in compression after 1000, 3000, and 8000 hours of aging. From the results of these tests, Kshirsagar found that it is evident that wrapped concrete cylinders (but not the concrete itself) were deteriorated under exposure to either hot liquid media or extended freeze-thaw cycling. He noted that a combination of moisture and elevated temperature was clearly more damaging than freeze-thaw cycling, and the strengthening effects of the FRP wrap were essentially lost after 3000 hours of aging.

In another study at West Virginia University, Javed [6] researched concrete beams externally bonded with carbon fiber tow sheets. Her objective was to study the stiffness of the wrapped concrete beams and bond pull-off strength under accelerated aging conditions. The reinforced concrete beams were $5 \mathrm{ft} \times 4$ in $x$ 6in and were tested as cantilever beams with an overhang of $3 \mathrm{ft} 9 \mathrm{in}$. They were designed as under-reinforced beams to ensure ductile flexural failure. The beams were reinforced using two \#3 bars (Grade 60) in the compression zone as well as the tension zone. Shear stirrups (\#3-Grade 40) were placed 6 inches center-to-center. Two longitudinal tow sheets and one transverse tow sheet were applied along the tension side. The test specimens for bond strength studies consisted of applying a 12" x 1" tow sheet strip, with fibers running along the length of the strip, to one face of a concrete cube and a second strip to the opposite face. Upon application, the strips were coated with epoxy and bonded to the cubes over a length of 4 and 6 inches. The externally bonded concrete beams and bond pull-off specimens were aged by placing them in an environmental chamber. While in 
the chamber, they were soaked in water as well as solutions having $\mathrm{pH}$ levels of 13 and 3 , and endured different levels of humidity and temperature.

The externally bonded beams and bond pull-off strength specimens were tested by Javed [6] after aging them for 5,15 , and 25 cycles. The study concluded that the beams aged under acidic and alkaline environments behaved differently from the hygrothermally aged beams. In all the beams aged for 5 cycles, an initial increase in stiffness was noted with respect to the control beams. On aging for 15 cycles the stiffness dropped to that of the control beams. However beams aged under acidic and alkaline conditions showed an increase in stiffness on further aging. Such increases are attributed, typically, to better cure of adhesives. However, hygrothermally aged beams showed about $17 \%$ decrease in stiffness before concrete cracking. It was stated that the behavior of the beams can be attributed to the behavior of the epoxy adhesive in different environments.

The bond pull-off tests revealed that the bond pull-off stress in the unaged 6-inch long specimens was found to be about $52 \%$ of the bond stress of the 4 -inch long specimen. The $48 \%$ reduction in bond stress is attributed to the parabolic distribution of bond force over a certain length beyond which it becomes zero. On aging for 25 cycles, the bond stress in the 6-inch long specimen did not change with respect to the control specimens. Whereas in the 4-inch long specimen, the ratio of bond stress in aged and unaged specimens at variable and constant temperature was about 0.71 and 0.80 respectively. Such bond stress variation can be explained from the view point of critical importance of bond length during aging, i.e., excess bond length over critical bond length can help adequate bond stress transfer even though partial degradation of bond length may take place under aging. 
Katsuki et al [10] performed a study on lap joint strength, adhesion strength, and bond strength of continuous fiber(CF) sheets on concrete in terms of long term durability. They concluded that a reduction in adhesive strength and lap joint division strength by the interface degradation of concrete and CF sheets was not observed. Rather, the strength degradation appeared to initiate in the concrete at the interface. The destruction of the test specimens also occurred in the base concrete. The bond strength durability tests concluded that degradation of the adhesive interface under dry and wet cycling was evident.

A recent study in Canada [3] focused on the effects of freeze-thaw on the bond between FRP sheets and concrete. Both glass and carbon fiber sheets were used. Beams were strengthened with the sheets and subjected to 50,150 , or 300 cycles of freeze-thaw exposure. At the end of exposure, the beams were tested to failure in 4 point bending. All of the beams failed with the same failure mode i.e., peeling of the bottom layer of concrete off the beam. The tests concluded that freeze-thaw action did not cause any degradation to the bond at the concrete/FRP interface. This conclusion was further reinforced from a similar study [19], in the United States of America.

Two previous studies, Armstrong [1] and Kinloch [11], examined the effect of absorbed water on adhesive bonding in CFRP composites. They concluded that the exposure of adhesive bond to water does not alter the effectiveness of bond to perform the necessary task to which it is entitled, i.e. no degradation was found. 


\subsection{Conclusions}

The effects that moisture, freeze-thaw, acid, alkaline, and temperature have on the bond characteristics will be studied through this research effort. Little is known about the primers and adhesives that generate bond forces, and because there are so many different adhesives and primers it is difficult to tell which ones are the best for different substrates. Since concrete and fiber strengths are well established, the hybrid (concrete + fibers) strength is dependent on the bond strength. There has been very little research done in this area, but it is becoming more and more of an issue leading to greater attention. By exposing the primer and adhesives to harsh environments (such as acid, alkaline, freeze-

thaw, hygrothermal, and water), we can determine the magnitude of bond strength degradation. These environmental factors have already proven to be detrimental to glass fibers [21], therefore we need to determine if any effects are occurring along the bond line so as to be able to predict a service life for the composite. With just a few studies done in this area, it is still uncertain what is really happening in the interfacial bond. Additional research has to be carried out to develop the reasoning on how and why the bond performs as it does. Therefore, the major thrust of this research is to help get us closer to developing sound engineering reasoning for bond degradation and also to develop bond stress reduction factors for design purposes under harsh environments. 


\section{Chapter 3}

\section{Materials}

\subsection{Introduction}

When studying the bond behavior of FRP and concrete, it is important to understand the materials that are involved. This is especially true about the adhesive and primer. There are many different types of adhesives that are used as bonding agents that may react differently under different environments. The following chapter explains each material that was used in this study.

\subsection{Concrete}

In order to save money and time, the cubes used in this research were constructed from two different types of concrete. One was a pea gravel and the other was of $1 / 2$ in. aggregate size. Both were supplied by Hoy REDI-MIX, Morgantown, WV, and were of about 4000 psi strength. The concrete was poured in two batches. The first specimens were used for the five month and nine month durations whereas the specimens used for the shorter durations were poured a few months later. The form work was removed about 5 days after pouring and the cubes were stacked together and wet burlap and plastic were placed over them for 28 day curing. The average compressive strength of the cylinders from the two batches was 4535 psi. 
Table 3.1 Compressive strength of concrete cylinders

\begin{tabular}{|c|c|}
\hline Batch & Compressive strength \\
\hline$\# 1$ & $4450 \mathrm{psi}$ \\
\hline$\# 2$ & $4620 \mathrm{psi}$ \\
\hline
\end{tabular}

\subsection{Carbon and Glass Fibers}

The carbon fiber tow sheet came from a Japanese company known as Tonen Corp. It was of type FT-C-20 and had unidirectional fibers supported on a paper backing. The fibers behave linear-elastically unto failure. Some properties of CFRP are: [6,22]

Design thickness of a single ply: $\quad .0043$ inches $(0.11 \mathrm{~mm})$

Tensile strength: $\quad 2.2 \mathrm{kip} / \mathrm{inch}(382 \mathrm{~N} / \mathrm{mm})$

Tensile Modulus: $\quad 33 \mathrm{Msi}\left(23.03 \times 10^{4} \mathrm{~N} / \mathrm{mm}^{2}\right)$

Ultimate strain: $\quad 1.5 \%$ or .015

Density: $\quad 0.056 \mathrm{lbs} / \mathrm{in}^{3}$

Shear Modulus: $\quad 7687 \mathrm{ksi}$

Poisson Ratio: $\quad .28$

The carbon fibers are resistant to moisture, some solvents, bases and weak acids. 
The glass fabric was provided by Master Builders of Cleveland, OH. It was of unidirectional fibers and had no backing. The fibers behave linear-elastically unto failure. Some properties of GFRP are: [6,22]

Design thickness of a single ply: $\quad .0235$ inches $(0.60 \mathrm{~mm})$

Tensile strength:

Tensile Modulus:

Ultimate strain:

Density:

Shear Modulus:

Poisson Ratio:
$76.9 \mathrm{ksi}$

$3770 \mathrm{ksi}$

$2-3 \%$

$0.063 \mathrm{lbs} / \mathrm{in}^{3}$

$1450 \mathrm{ksi}$

.28

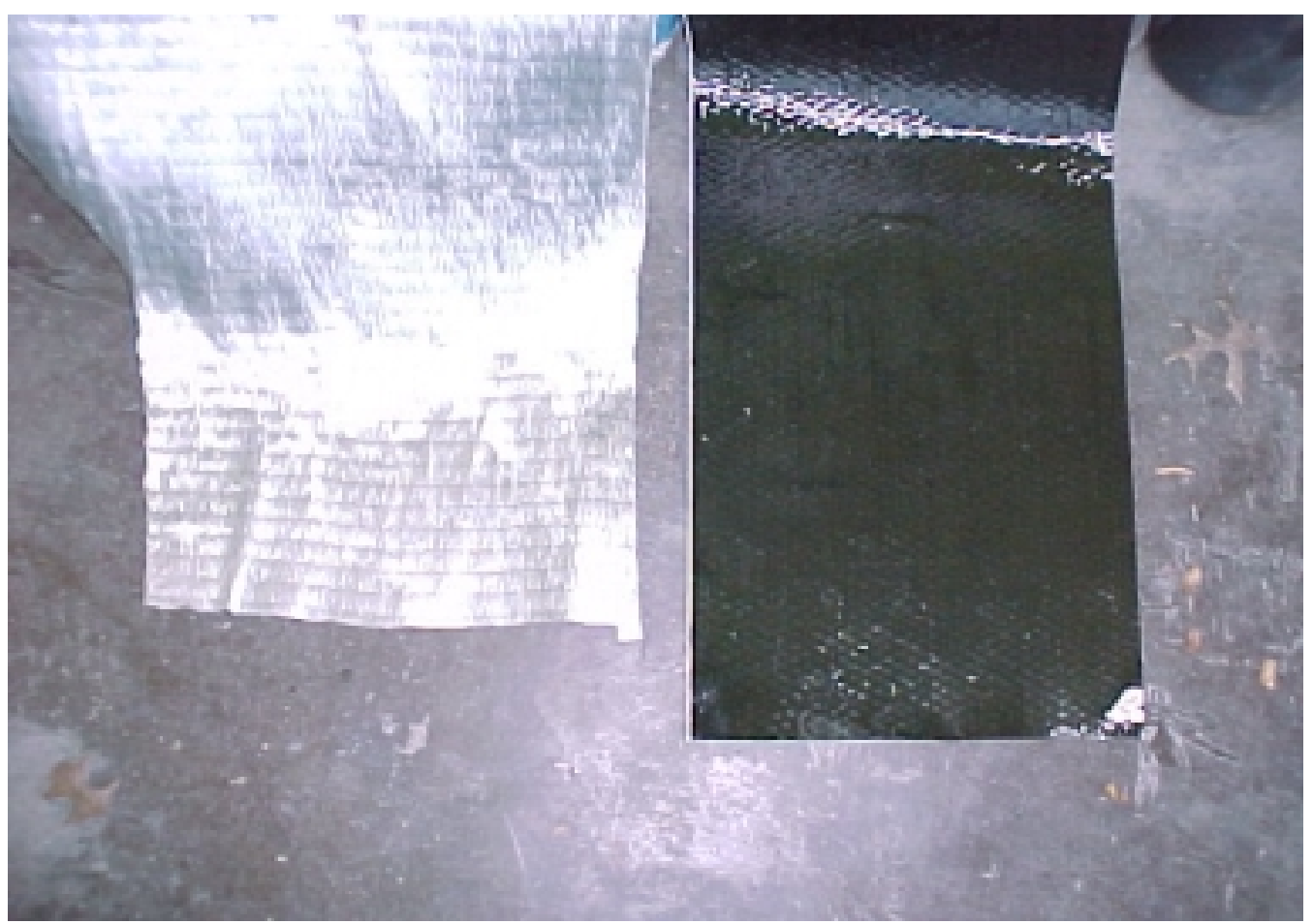

Figure 3.1 GFRP (left) and CFRP (right) 


\subsection{Primer and Adhesive}

The adhesive used was known as the Mbrace system. The manufacturer, Master Builders, provided this material. The primer was a two-part primer with a mix ratio of part A to part B of 3:1 by weight or volume. It was applied to the concrete surface and allowed to cure for 24 hours. The adhesive was also a two-part mixture with the mix ratio being the same as that of the primer. It was applied to the concrete surface as well as the fabric the day after the primer was applied. The method used to apply the material was suggested by the manufacturer. The proper procedure for application of primer, epoxy and FRP is given in appendix A.

For a detailed explanation of the materials that make up the adhesive, including properties and curing effects, refer to the manufacturer's notes in appendix A. 


\section{Chapter 4}

\section{Test Specimens and Experimental Set-Up}

\subsection{Introduction}

The following chapter explains in detail how the specimens were constructed and tested, and the conditioning scheme of test specimens under hygro-thermal fluctuations. The specimens were fabricated and tested in the Major Units Laboratory at WVU. It should be noted that each specimen is not an exact duplicate of the next due to varying surface conditions and dimensions. The most severely distorted specimens were discarded.

\subsection{Specimen Description and Preparation}

A typical specimen consisted of two $4 \times 4 \times 4$ inch concrete cubes connected by two strips of FRP composite. The cubes were constructed using formwork that consisted of long steel plates and angles (see Figure 4.1). Wooden forms were also constructed so as to produce more cubes per pour. The plates were laid flat on the floor and the 4 " high angles were laid along them. At four inch intervals, $4 \times 4$ inch wooden spacers were placed between the angles as to separate one cube from the next (Figure 4.1). After these cubes were poured and allowed to cure for 28 days, they were then paired up and numbered (two that resembled each other the most in size would make up one pair). Some cubes were discarded because they were over-sized due to the movement of the wooden spacers during vibration of the concrete. Also, upon removing the wooden 
forms, it was discovered that about half of the specimens were unacceptable, therefore these forms were not used in future pours.

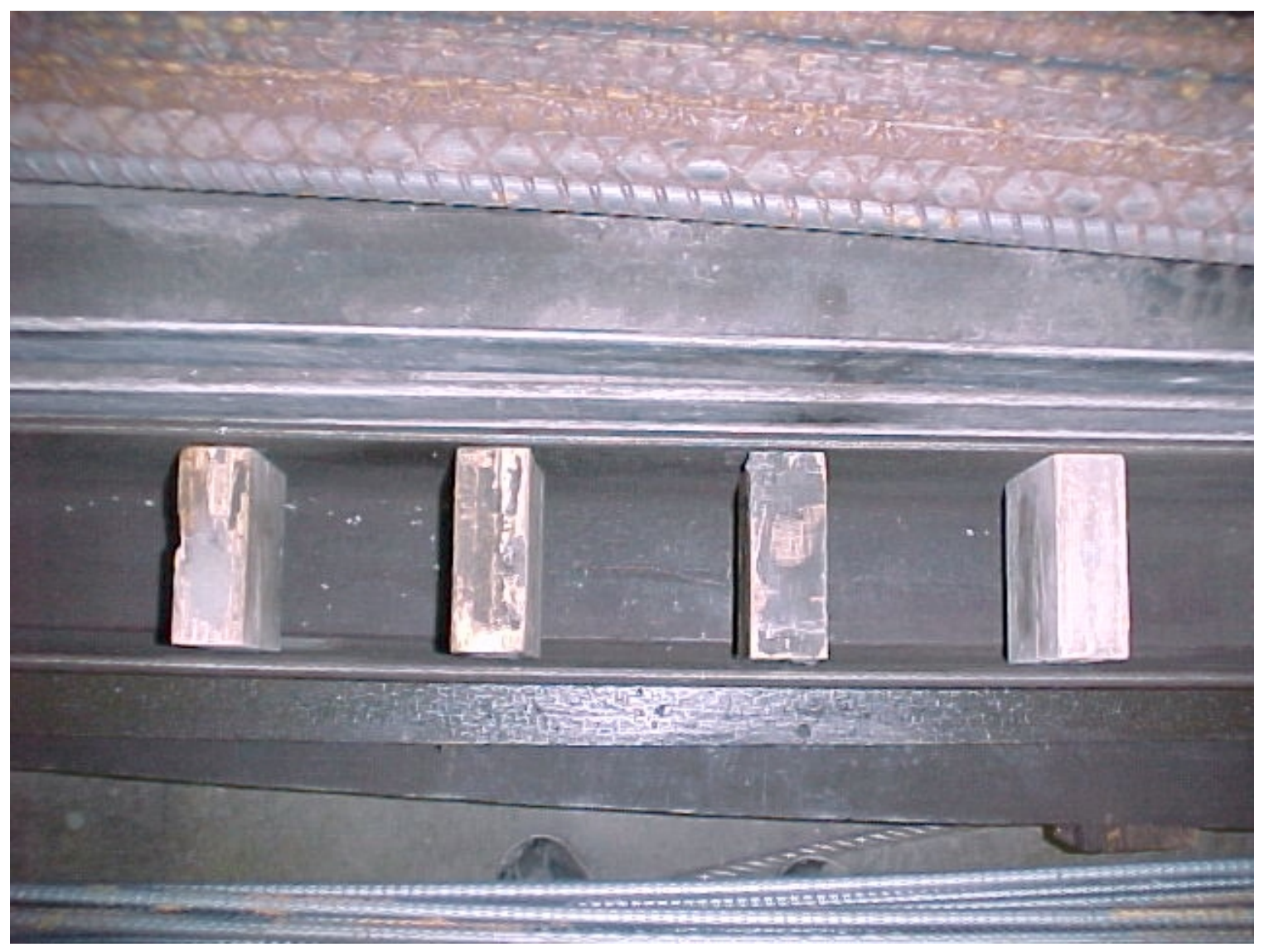

Figure 4.1 Formwork for concrete cubes

\subsubsection{Attachment of Strip}

The next step was the application of the primer. The primer, which was a twopart mix ratio of 3:1 (by volume as well as weight), was mixed and applied to all six sides of the cube using a paint roller. The cubes were placed on a sheet of wax paper and left to dry for 24 hours. After this, the strips were ready to be cut from the sheets of fiber. A sheet of carbon or glass was laid out on a piece of wax paper. Using a paint roller, we 
would then apply a very thin coat of resin to one side of the sheet. This was done for two reasons: 1)It made the material easier to handle and cut; and 2) upon application of the epoxy, it kept the fibers from fraying out. For example, when using glass a thin coat was needed on both sides of the sheet due to the thickness of the material. After the resin on the sheet was cured (approx. 24 hours), the strips were cut out using scissors. Each strip was 10 inches long and approximately $1 \frac{1}{4}$ " wide.

The resin system (resin \& epoxy) was now ready to be made. This would consist of the two-part mixture (parts A \& B) with a weight or volume ratio of 3:1 because of near identical specific gravity of parts A and B. The mixture was stirred for five minutes. Using a paint brush, the adhesive was then applied to the ends of the strip and onto the cubes where the strip was to be bonded. Another strip was placed on the opposite side of the cubes in the same manner. The clearance between the cubes was about $6 "$ and therefore about a 2" bond was allowed on each end (Figure 4.2). While curing took place (24-48 hours), pressure was required to hold the strip in place. Our first method for doing this was to apply some weights (small steel plates) to the bond area. However, this would mean that we would have to return the next day and flip the specimen over and apply the second strip; thus requiring two days and another mixture of epoxy to complete the process. There were also only a limited number of weights available for this kind of manual operation. Therefore, in order to apply pressure to both sides of the specimens at the same time, we laid the specimens on their sides and butted them against each other (with an elastomeric pad between them) and applied a giant clamp to the ends (Figure 4.3). Not only did this allow us to create more specimens but it also helped us to complete the process in one day. As the pressure was applied some strips did move a 
little which resulted in having slight variation in bond lengths of exactly two inches. Therefore, both methods for applying pressure were used and it was discovered that as long as some pressure was applied, slight variation in bond length did not affect the bond strength.

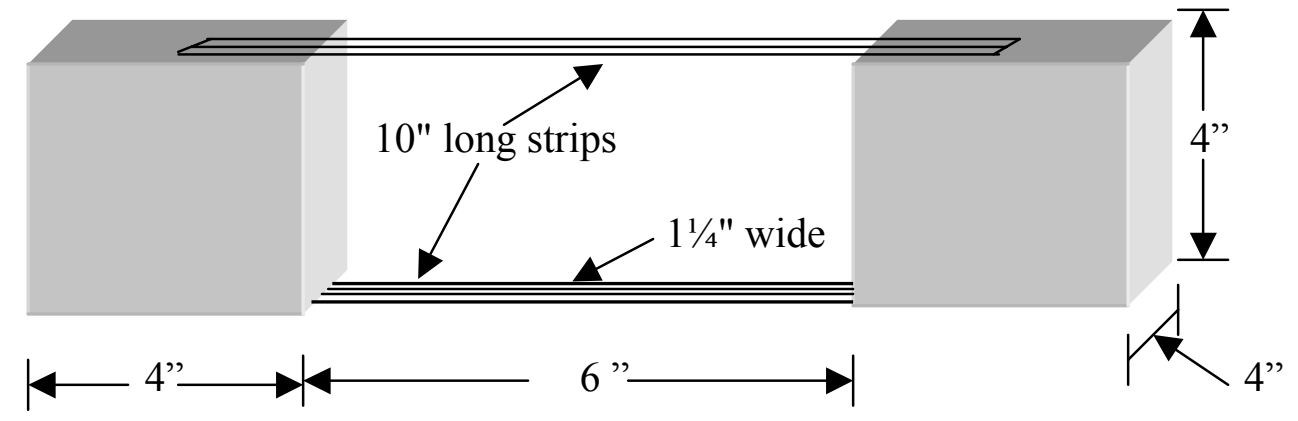

Figure 4.2 Typical dimensions of specimen

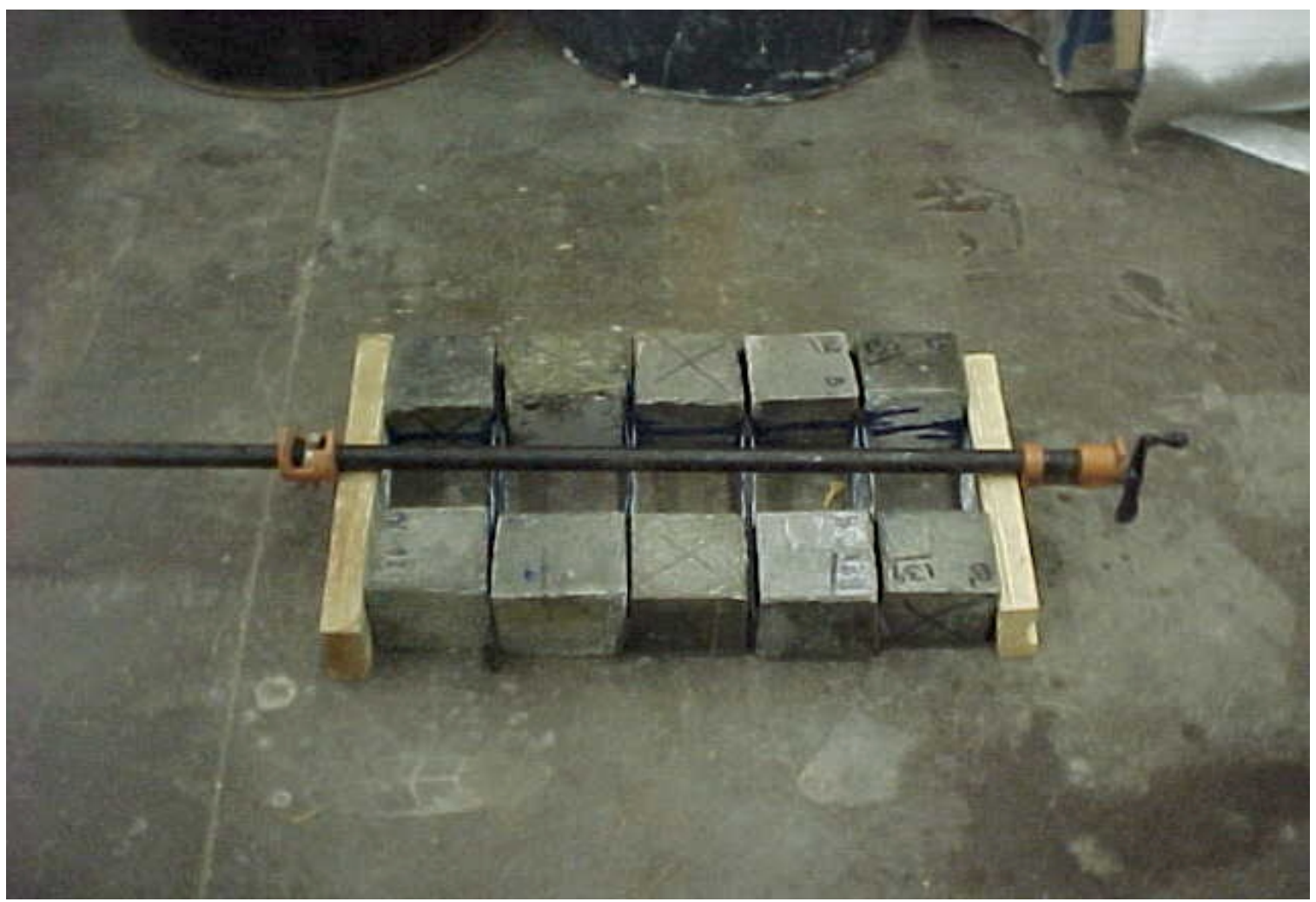

Figure 4.3 Application of pressure to specimens during curing 


\subsection{Instrumentation}

In order to find the most effective way to apply stress and distribute it along the bonded area, two strain gages were placed one behind the other with the first one starting at the edge of the strip bonded to concrete(Figure 4.4). The gages, type CEA-06250UW-350, were from Micro-Measurements(MM). The lead wires (type DFV-326, also from $\mathrm{MM}$ ) were then connected to the gages and the specimen was then transported to the testing machine.

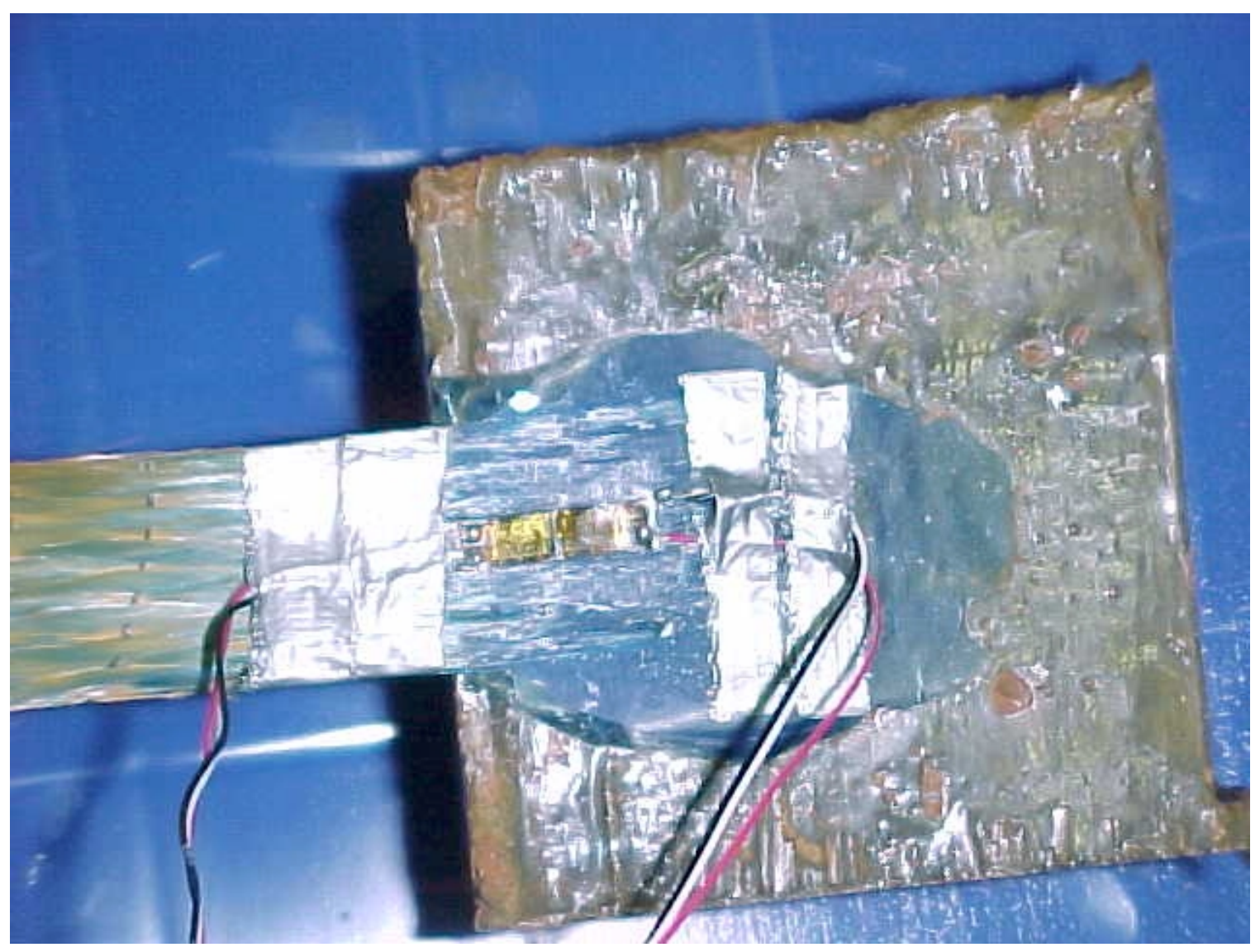

Figure 4.4 Gage detail

\subsection{Testing Set-Up}

Special grips, designed and built to fit over each cube so that the specimens could be loaded in the Baldwin machine(Figure 4.5), were placed on the specimens (Figures 4.6 
and 4.7). The grip was designed so that the shafts that were placed into the machine could rotate, thus preventing any eccentricity during loading. The grips, along with the specimen, were then placed into the loading machine (Figure 4.8-Note: the figure depicts an apparatus used for sustaining a load which is discussed in section 4.5 , this apparatus was removed prior to testing). The lead wires that were connected to the strain gages were hooked up to strain indicators and the initial readings were taken. The load was then applied at a rate of approximately $2 \mathrm{lbs} . / \mathrm{sec}$.(this exact rate was not easily maintained due to the sensitivity of the machine). Strain readings were taken every 50 lbs. until failure occurred. The data were then recorded into Excel software so that graphs and charts could be produced to display the results.

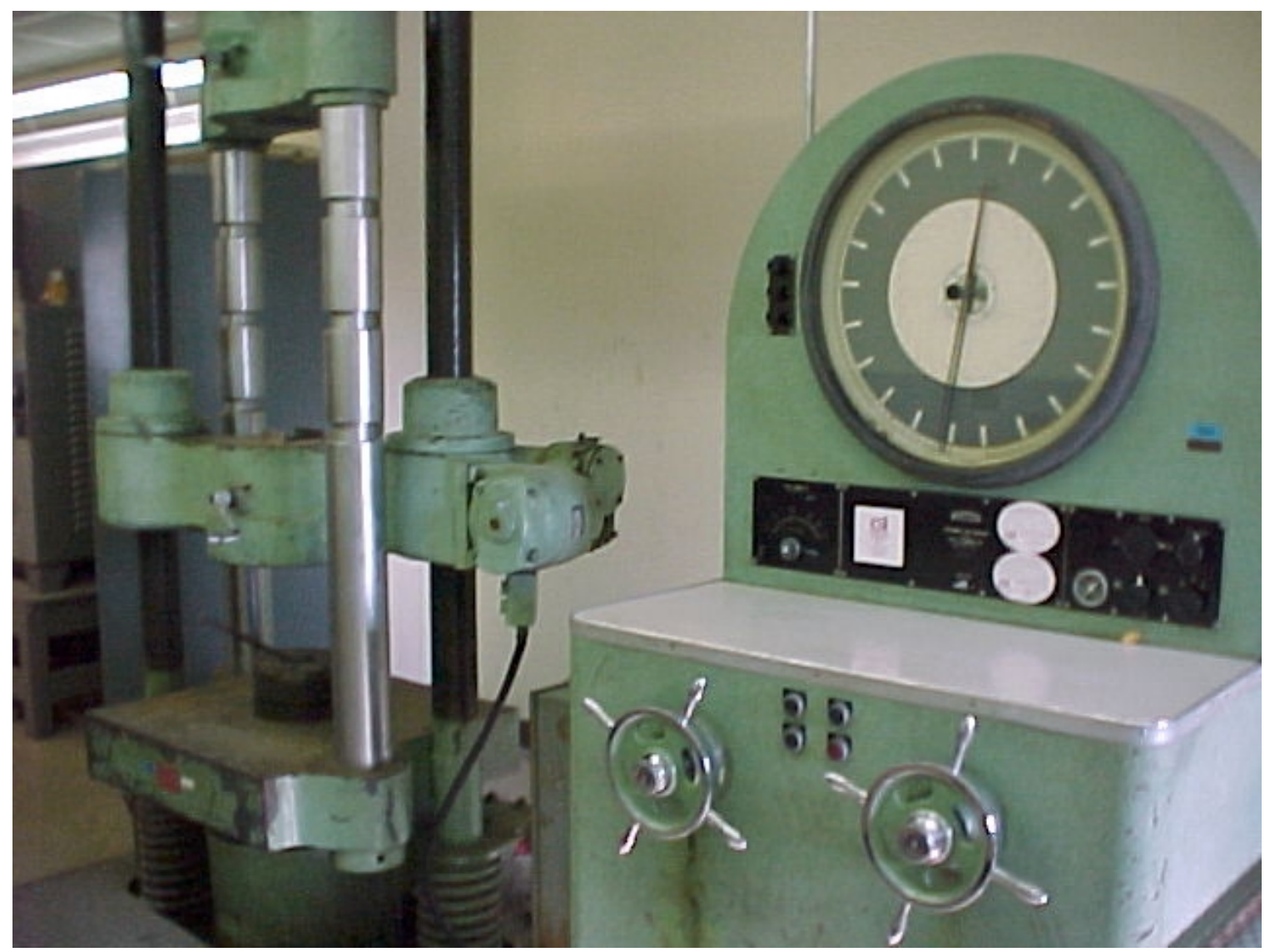

Figure 4.5 Baldwin Machine used for testing specimens 


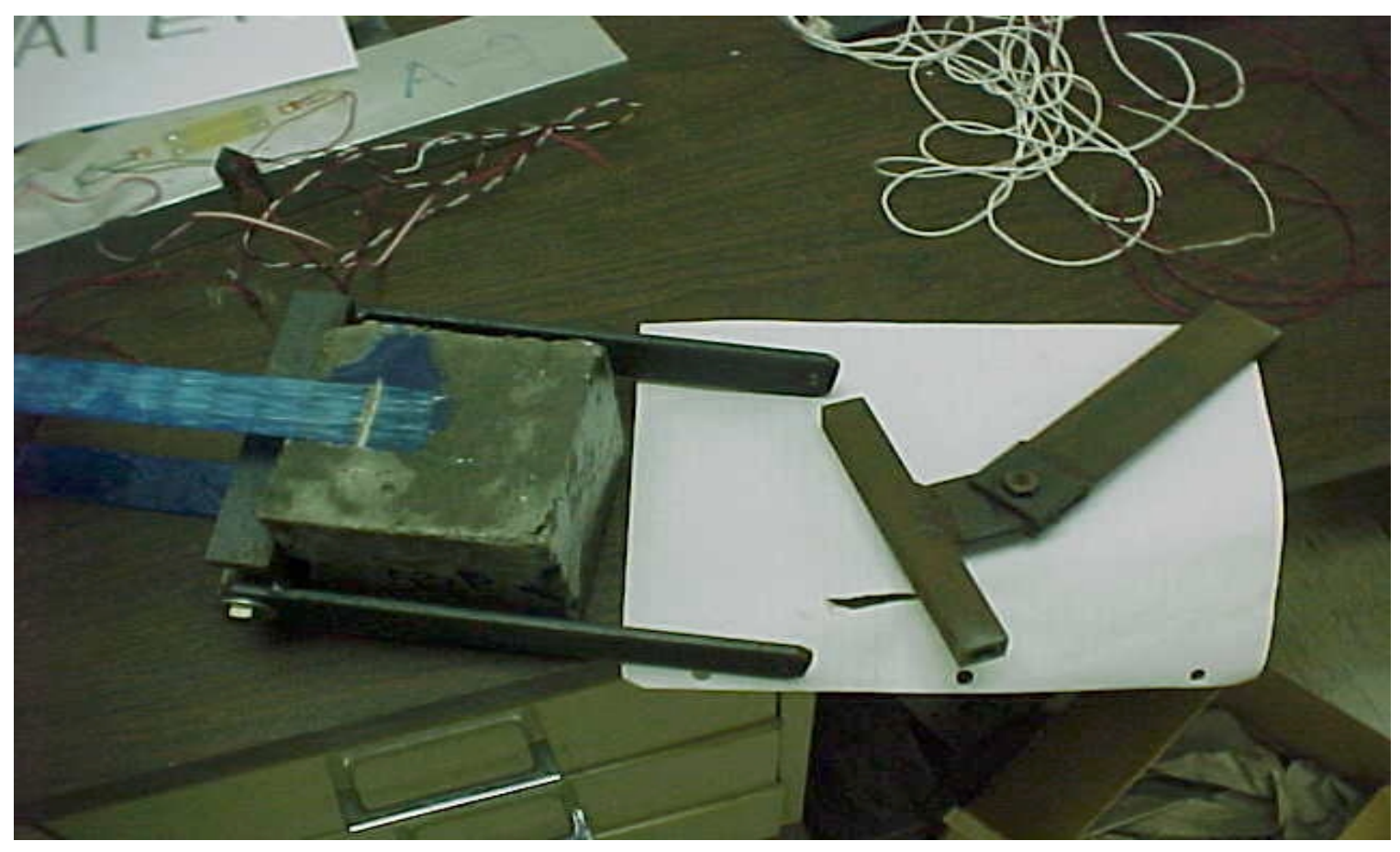

Figure 4.6 Grip detail

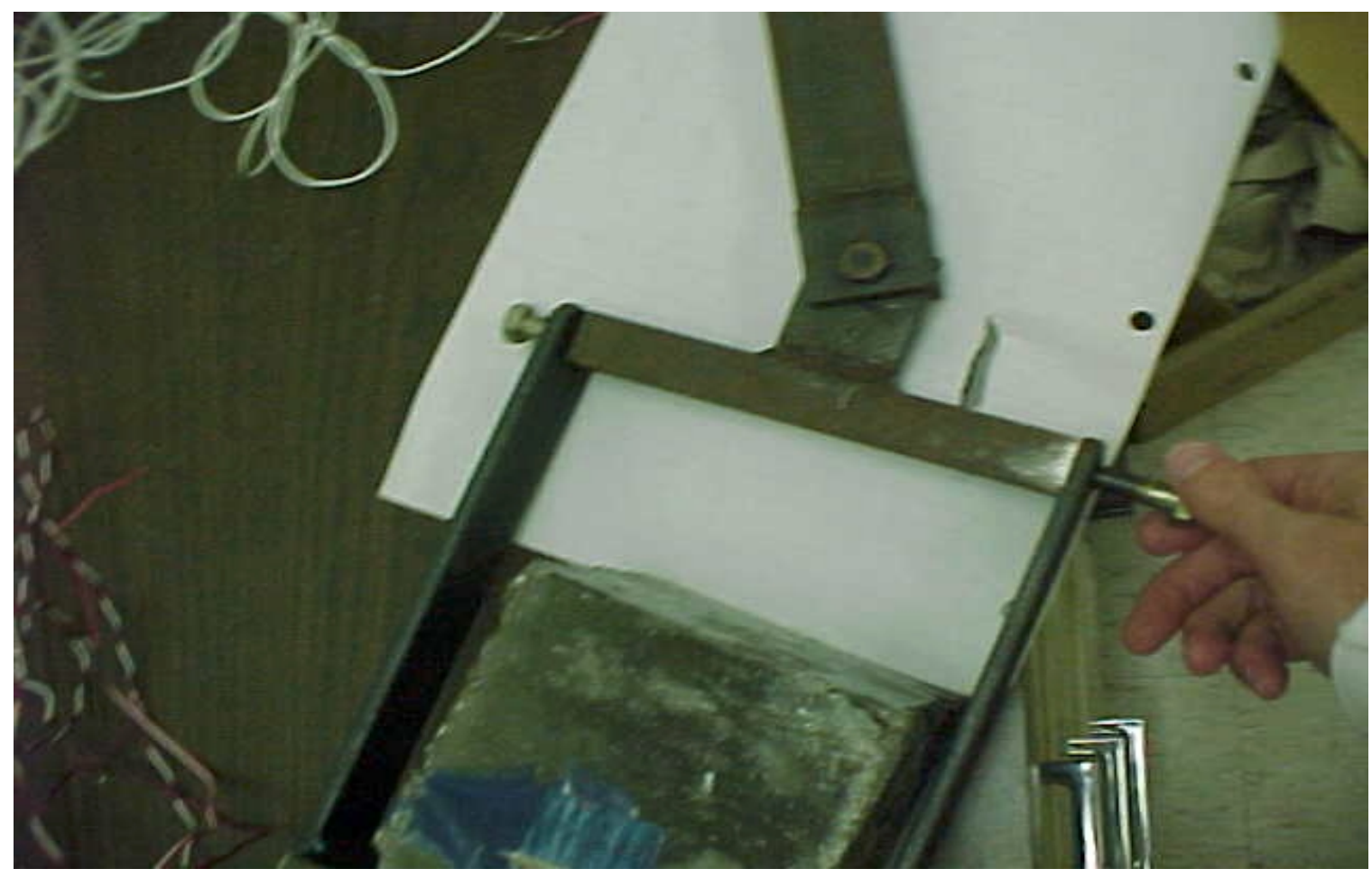

Figure 4.7 Application of grips to specimen 


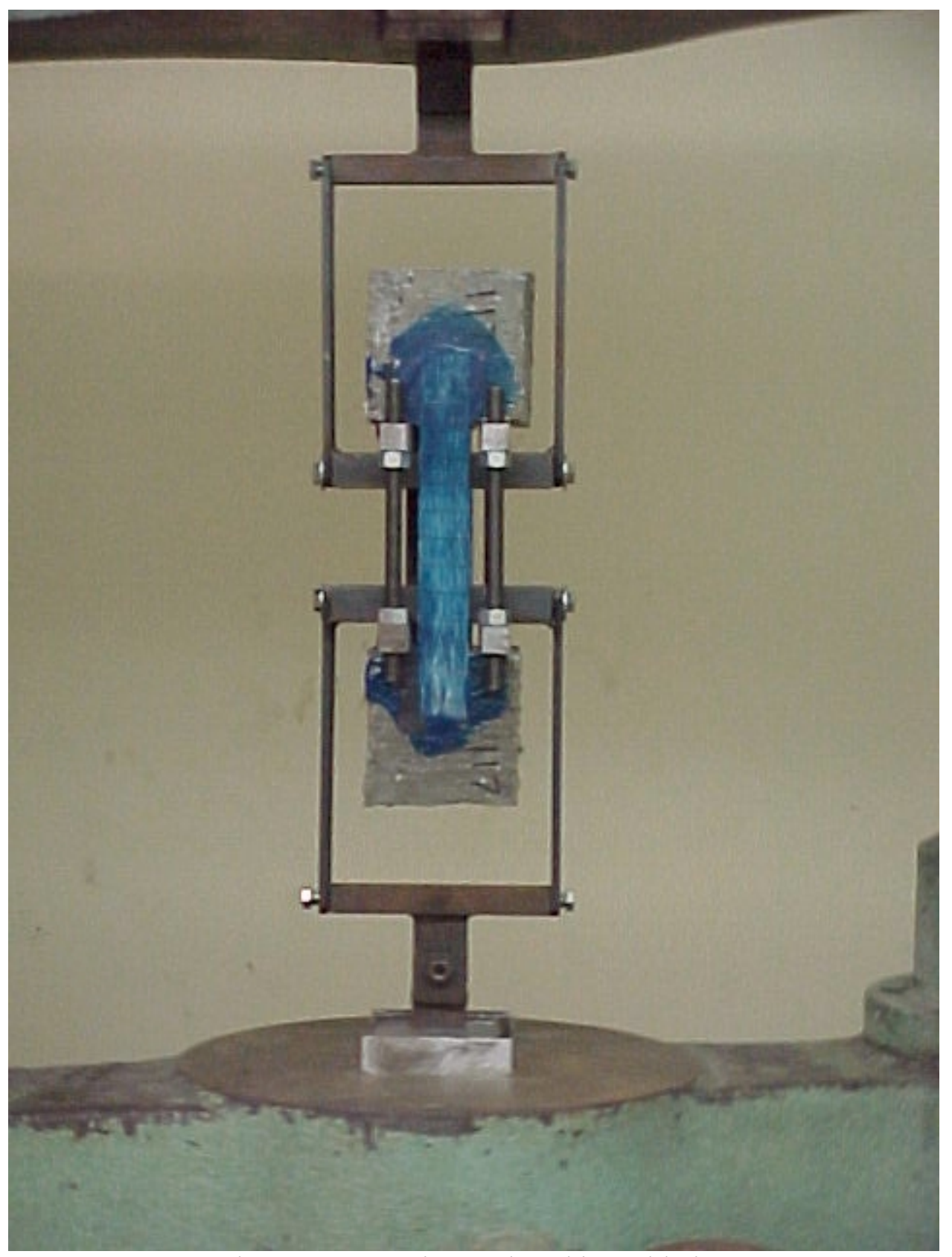

Figure 4.8 Specimen placed in Baldwin 


\subsection{Test Parameters/Conditioning}

After curing the adhesive, the specimens were each placed in a specific aging environment (see Table 4.1). These environments include $\mathrm{pH} 3$ at room temperature, $\mathrm{pH}$ 13 at room temperature, $100 \%$ moisture (soaking) at room temperature, freeze-thaw effect through environmental chamber, $\mathrm{pH} 3$ \& chamber, $\mathrm{pH} 13$ \& chamber and natural aging (Table 4.1). The chamber takes 5 days to complete one cycle. This cycle goes through different humidity and temperature levels (Table 4.2). The specimens were conditioned for 1 month, 3 months, 5 months and 9 months. Specimens conditioned for 1 month and 3 months were under a sustained stress whereas the 5 and 9 month specimens were not. To perform a sustained stress, threaded rods with nuts were put through steel shafts that were placed between the cubes. Then the specimens were loaded in the Baldwin machine to a level of $500 \mathrm{lbs}(100 \mathrm{psi})$. When the machine reached $500 \mathrm{lbs}$. the nuts were tightened and the machine was shut down thus maintaining the load (Fig. 4.9).

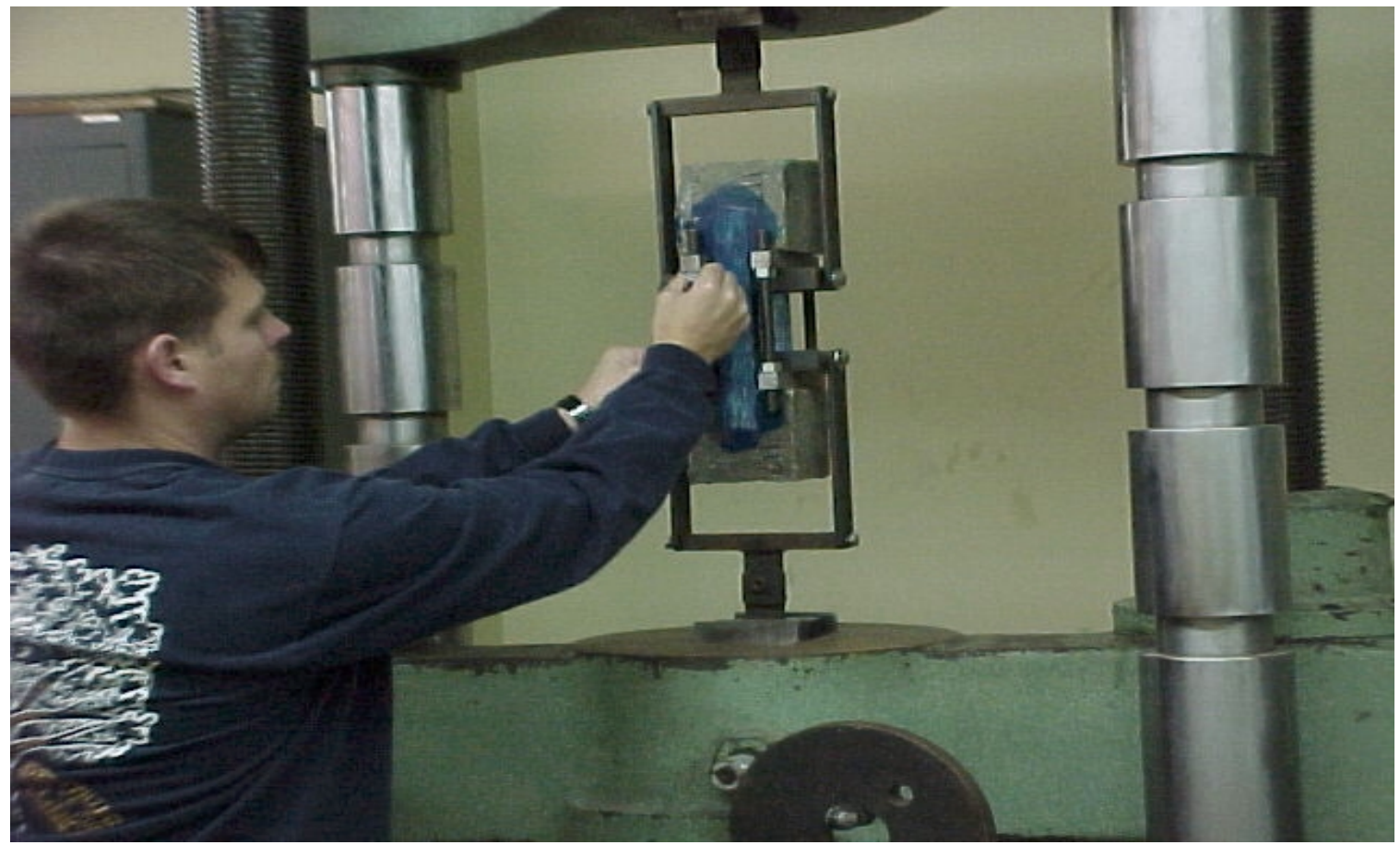

Figure 4.9 Application of sustained load 
Table 4.1 Conditioning Scheme

\begin{tabular}{|c|c|c|}
\hline Specimen & Conditioning & Duration \\
\hline $1-2,18,120$ & Control & 0 days \\
\hline $6-7,19-20$ & Chamber & 9 months \\
\hline $8-9,29-30$ & Chamber/pH 13 & 5 months \\
\hline $12,15,26-27$ & $100 \%$ moisture & 9 months \\
\hline $13-14,21-22$ & Chamber/pH 13 & 9 months \\
\hline $16,32,47,48$ & Chamber/pH 3 & 5 months \\
\hline $28,31,35-36$ & Chamber/pH 3 & 9 months \\
\hline $37-38,51-52$ & $100 \%$ moisture & 5 months \\
\hline $33-34,49-50$ & Chamber & 5 months \\
\hline $41-44,57-60$ & $\begin{array}{l}\text { Natural Aging } \\
\text { (outside) }\end{array}$ & 9 months \\
\hline $65-67,83-85$ & Chamber & 3 months \\
\hline 71,73-74,90,92,94 & Chamber/pH 13 & 3 months \\
\hline $69,70,86,87,89$ & Chamber/pH 3 & 3 months \\
\hline $63-64,72,80-82$ & Room Temp./pH 13 & 3 months \\
\hline $61-62,68,77-79$ & Room Temp./pH 3 & 3 months \\
\hline 102-104,115-117 & Chamber & 1 month \\
\hline $140,137,128,134-136$ & Chamber/pH 13 & 1 month \\
\hline $97-99,141,142,132$ & Chamber/pH 3 & 1 month \\
\hline $\begin{array}{l}109,105,113,118,93, \\
95\end{array}$ & Room Temp./pH 13 & 1 month \\
\hline $\begin{array}{l}101,107,112,121, \\
123\end{array}$ & Room Temp./pH 3 & 1 month \\
\hline
\end{tabular}


Aside from the accelerated aging, specimens were ( 4 carbon \& 4 glass) naturally aged. These were placed outside without a sustained load applied to them (Figure 4.11). We also had 6 control specimens ( 3 carbon $\& 3$ glass). These were used to develop the standard to which the aged specimens would be compared.

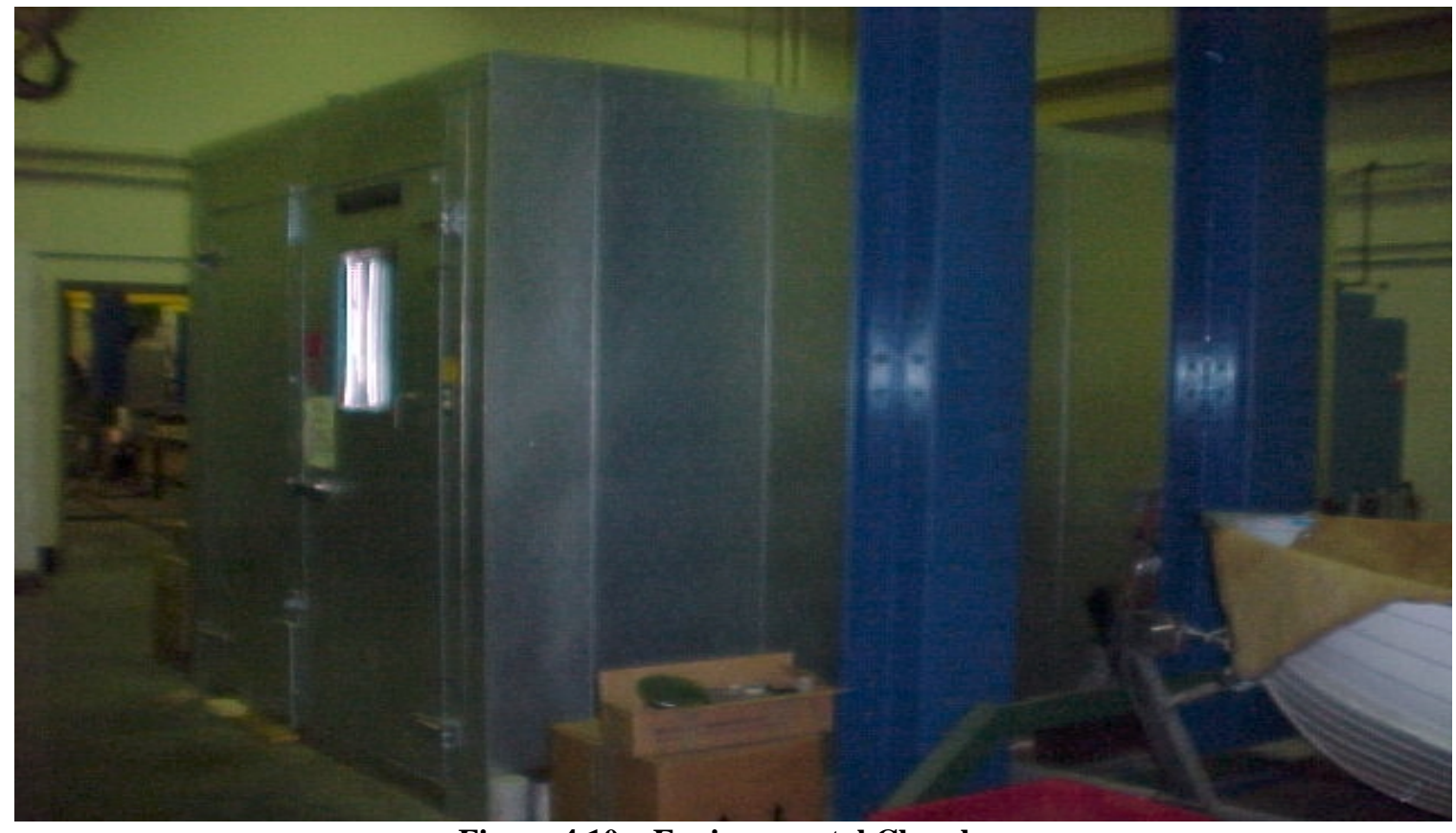

Figure 4.10a Environmental Chamber

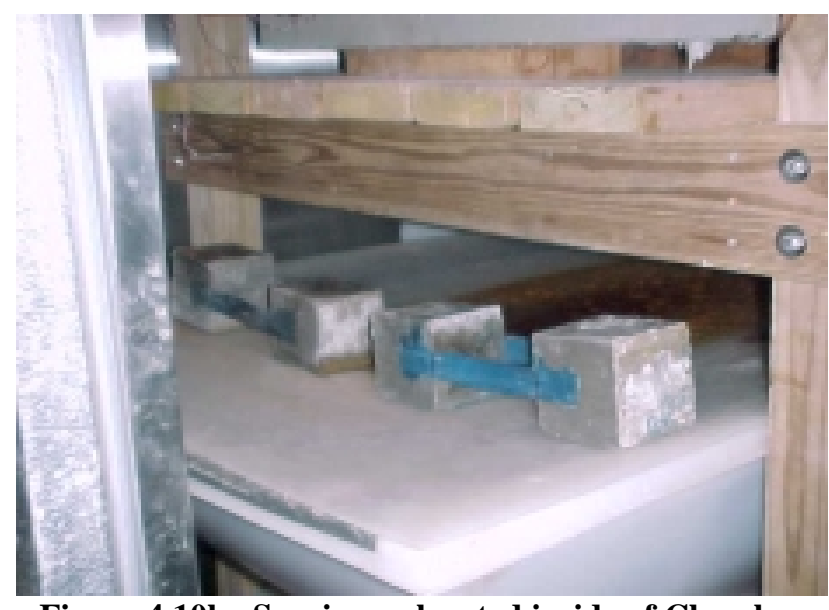

Figure 4.10b Specimens located inside of Chamber 
Table 4.2 Temperature and Humidity Cycle of Environmental Chamber

\begin{tabular}{|c|c|c|c|c|c|}
\hline \multirow{2}{*}{ Number of Hours } & \multicolumn{2}{|c|}{$\begin{array}{c}\text { Chamber Temperature } \\
\end{array}$} & \multicolumn{2}{c|}{$\begin{array}{c}\text { Avg. Chamber } \\
\text { Temperature } \\
\end{array}{ }^{\circ} \mathbf{F}$} & Humidity (\%) \\
\hline 1 & 69.8 to 109.4 & 21 to 43 & 89.6 & 32 & 0 to 95 \\
\hline 23 & 109.4 & 43 & 109.4 & 43 & 95 \\
\hline 1 & 109.4 to 120.2 & 43 to 49 & 114.8 & 46 & 95 to 10 \\
\hline 23 & 120.2 & 49 & 120.2 & 49 & 10 \\
\hline 1 & 120.2 to 71.6 & 49 to 22 & 95.9 & 35.5 & 10 to 95 \\
\hline 23 & 71.6 & 22 & 69.8 & 22 & 95 \\
\hline 4 & 71.6 to 12.2 & 22 to -11 & 41.9 & 5.5 & 95 to 0 \\
\hline 8 & 12.2 & -11 & 12.2 & -11 & 0 \\
\hline 3 & 12.2 to 109.4 & -11 to 43 & 60.8 & 16 & 0 to 95 \\
\hline 31 & 109.4 & 43 & 109.4 & 43 & 95 \\
\hline 3 & 109.4 to 69.8 & 43 to 21 & 89.6 & 32 & 95 to 0 \\
\hline \multicolumn{7}{r|}{ Average Chamber Temperature: $93.68^{\circ} \mathrm{F}\left(34.27^{\circ} \mathrm{C}\right)$} & \\
\hline
\end{tabular}

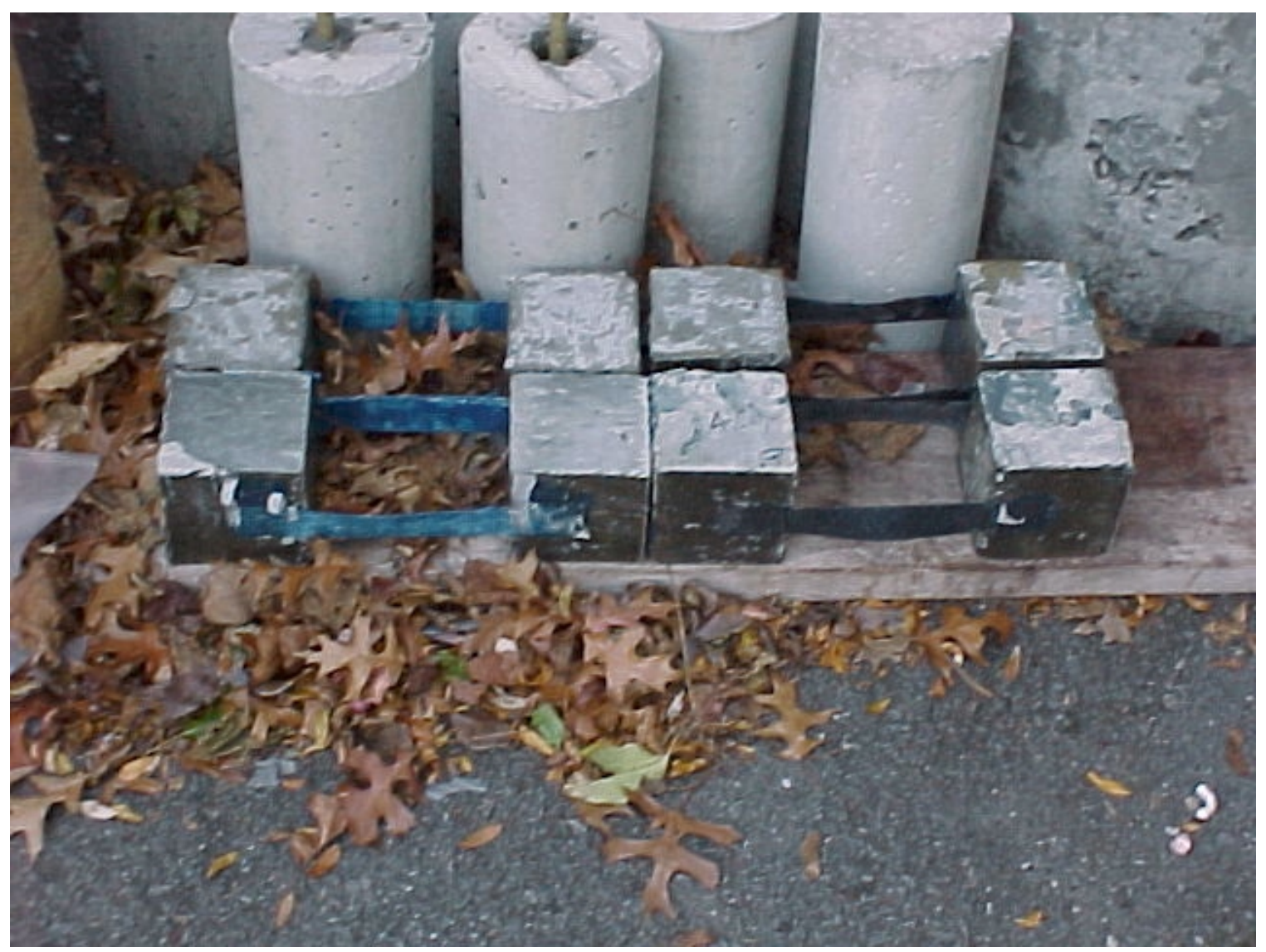

Figure 4.11 Natural Aging of Specimens 


\subsection{Beam Specimens}

Tests were also conducted using the Baldwin machine (Fig. 4.14) on small beam specimens. These specimens had been sitting at room temperature for at least 24 months. We immediately tested three samples and recorded the results. A couple of test samples were placed into the environmental chamber for future testing. The beam specimens were investigated to validate their bond behavior to the cube specimens. The carbon composite materials used for these specimens were identical. A carbon fiber strip that ran along one side of the beam connected two blocks made of mortar. The blocks were approximately 2 inches wide. The same "Mbrace" adhesive system was used for bonding the strips. On the opposite side was a hinge, which allowed the beam to become a determinate structure. Detailed drawings of these specimens are shown below (Figures $4.12 \& 4.13)$.

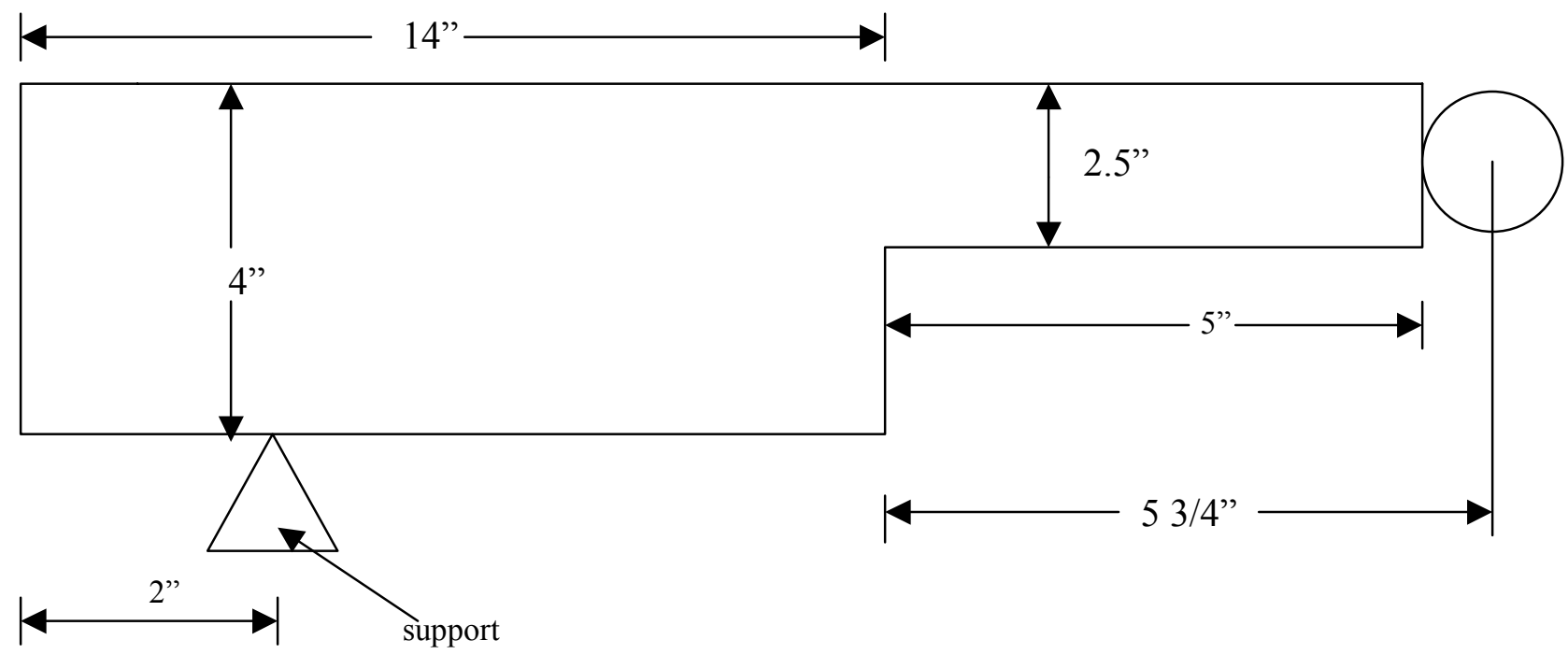

Figure 4.12 Dimensions of One Mortar Block 


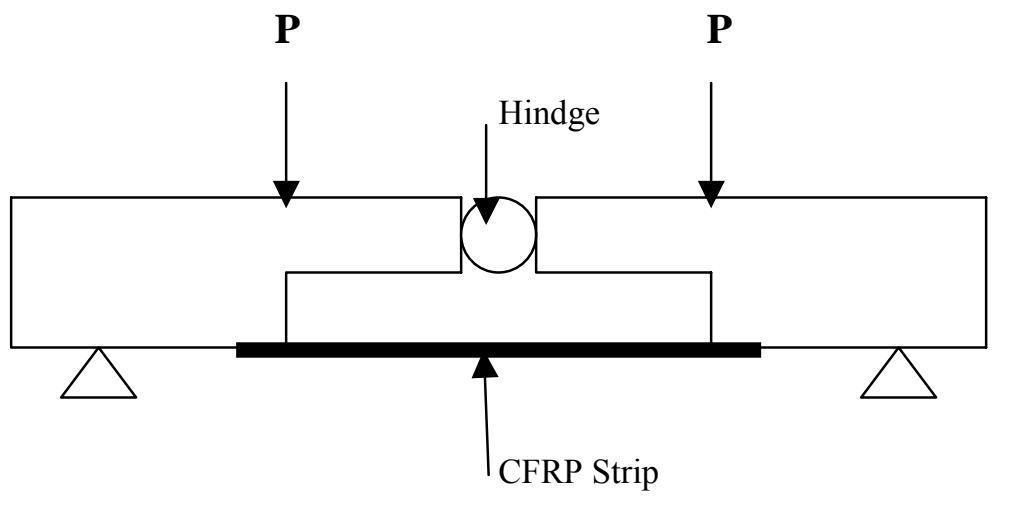

Figure a.

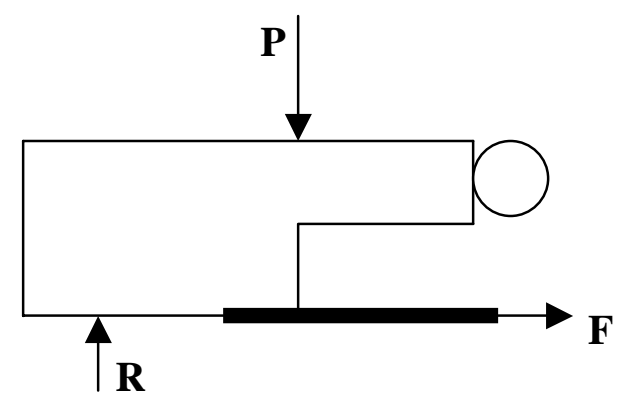

Figure b.

Figure 4.13 a/b Beam Specimen Details

\section{Force Equilibrium}

$\Sigma \mathrm{M}_{\text {hindge }}=5 \mathrm{P}+2.75 \mathrm{~F}-17 \mathrm{R}=0$

\section{Solving for $\mathbf{F}$ :}

$\mathrm{F}=(17 \mathrm{R}-5 \mathrm{P}) / 2.75$

$\mathrm{P}=\mathrm{R}$, therefore

$\mathrm{F}=12 \mathrm{P} / 2.75$

The force (F) is then divided by the bonded area, to get the bond stress. 


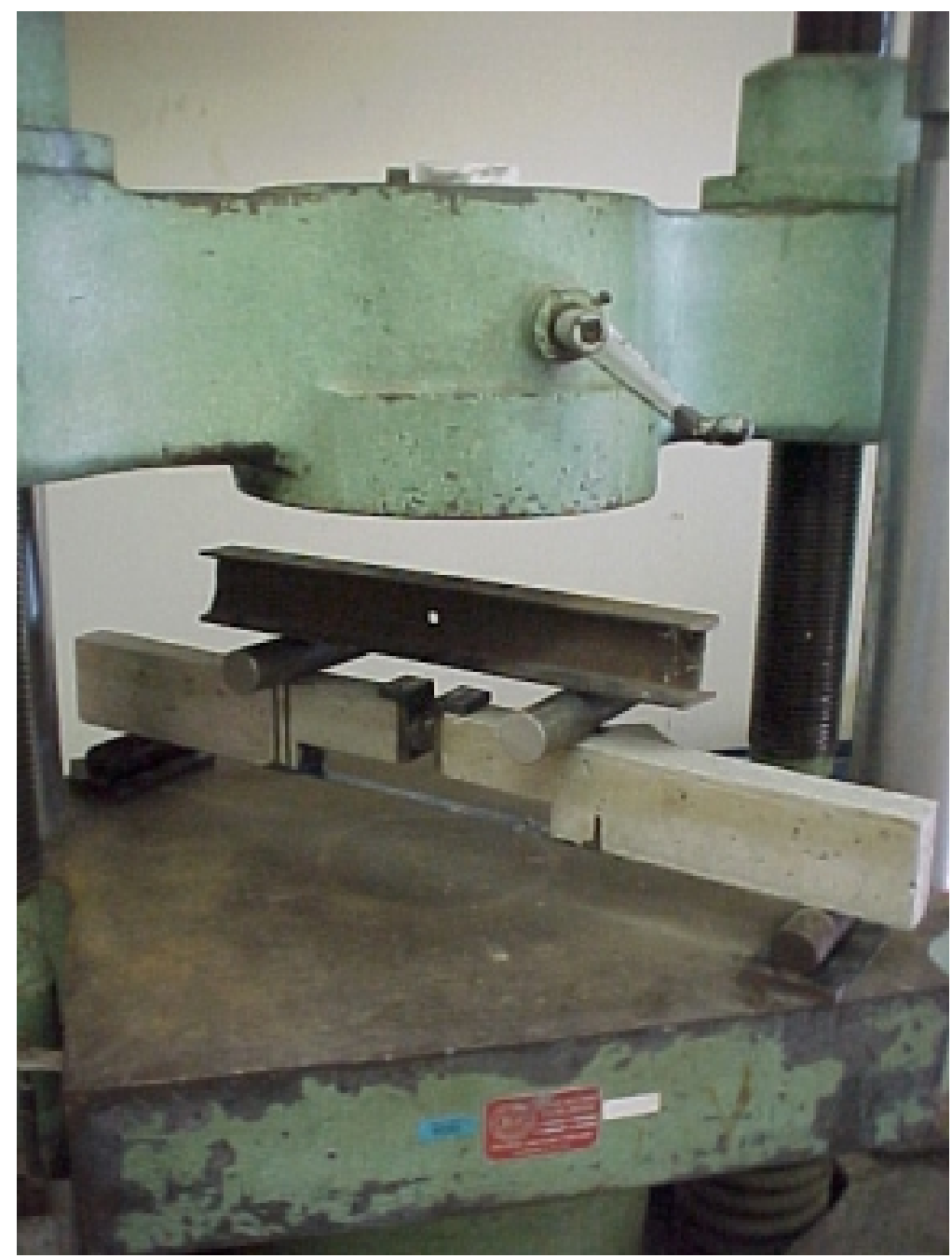

Figure 4.14 Test set-up for Beam Specimens

The following chapter (chapter 5) evaluates the test results under each aging condition and offers explanations to the outcomes, including failure modes. 


\section{Chapter 5}

\section{Experimental Results and Discussion}

\subsection{Introduction}

The following chapter presents the results that were found from the aged specimens as well as the unaged. The graphs show the distribution of strain along the bond length of composite strips. The strain readings were taken at different distances from the edge of the concrete block, along the bonded length of composite strips. Each graph shows the locations of strain gages with respect to the edge of the concrete block (i.e. where the bond force transfer begins). The bar charts depict the bond strength of the aged specimens. In all the charts and graphs, the bond strength of aged specimens is compared with the unaged specimens. The bond strength results are evaluated in this chapter in reference to their strain distribution. In addition, modes of failure have been identified and delineated, i.e. which failure mode occurs under what condition. Finally, test results are theoretically evaluated to provide proper engineering explanation for failure strains and bifurcation of stress-strain plots.

\subsection{Control (Unaged) Specimens}

The following graphs (Figures 5.1--5.5) show the strain distribution for the unaged glass and carbon specimens and these are designated as control specimens. The data from unaged specimens are used to compare with the data from the aged specimens to determine the bond strength degradation rate. Two GFRP and four CFRP unaged specimens were tested. From these results, a control graph for each material was derived. 
In case of bond tests using carbon fibers, two control graphs (Figures $5.5 \& 5.6$ ) were used. The average bond strength was 405 psi for glass and 480 psi for carbon. This was found by averaging the ultimate bond strength of the specimens.

\subsubsection{Glass-Control}

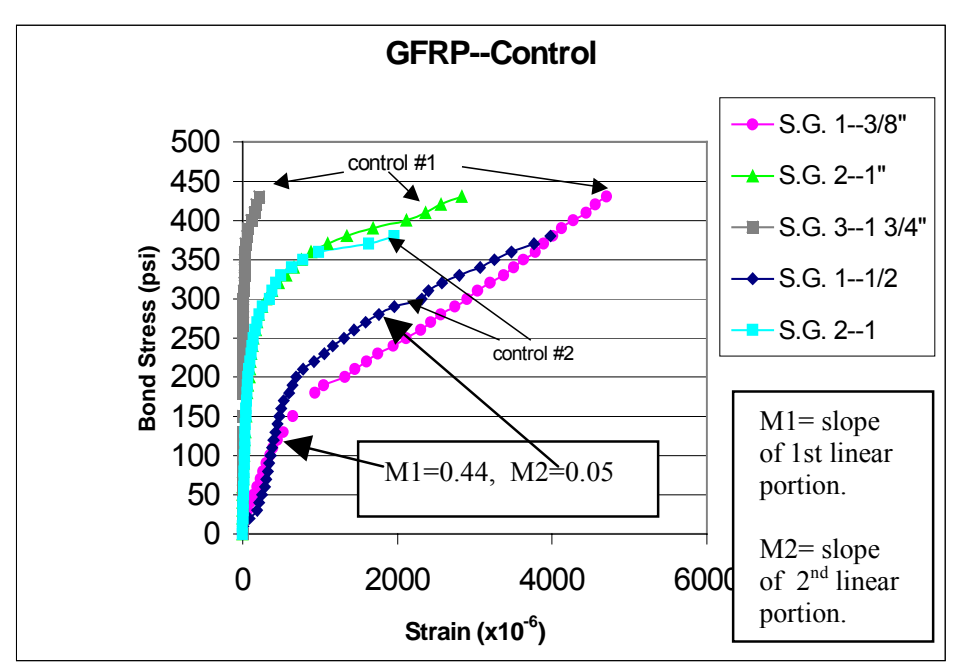

Figure 5.1 Strain Distribution for Control Specimens with Glass strips

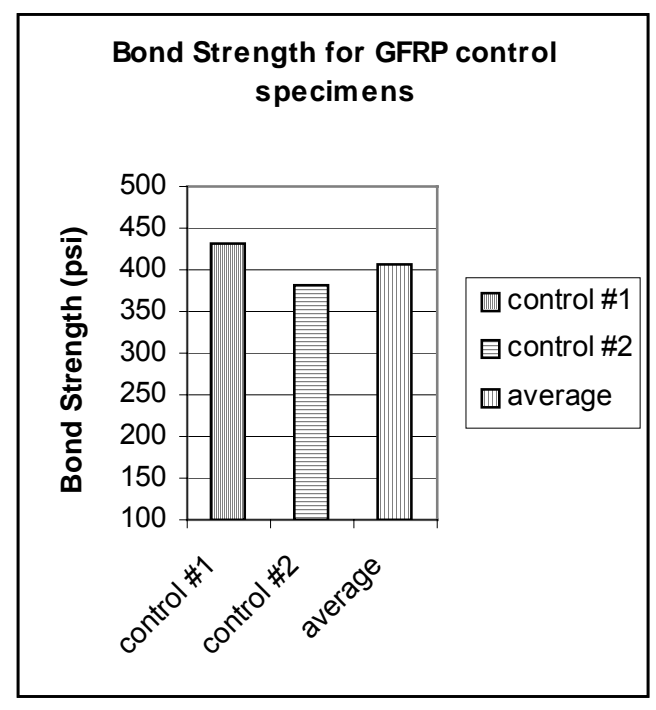

Figure 5.1a Bond Strength for GFRP control specimens

It can be seen in Figure 5.1 that the two strain gages located 1" from the edge are both in total agreement. Figure 5.2 displays strain as a function of location for the unaged GFRP specimens of Figure 5.1. These two gages came from separate specimens. Figures 5.3 and 5.4 show the placement of the gages. From control specimen \#1, the ultimate bond strength, found from dividing the force by the area, is $430 \mathrm{psi}$. The average bond strength of the two control specimens is 405 psi (Figure 5.1a). It can be seen that the change in slope occurs at lower stress levels for gages closest to the edge. The change in slope occurs around 200 psi for gages located approximately $1 / 2$ " from the beginning of the bond stress transfer location, and at about 300 psi for those located 1" 
back. This difference in stress level (200 vs. 300 psi) where change in slope is starting to happen, is attributed to stress concentration due to edge effects, i.e. near the edge of the test sample. However, the strain level where nonlinearity begins is about the same for all gages. The cause of this may be that the strip is delaminating causing the effective bond area to shift back until it propagates completely[14], or at least some bending effects start exerting on the bond line in addition to pure in-plane shear.

The slope of the lines in Figure 5.2 represents the rate at which the strain is propagating along the bond line. This is approximately 3683-3937 $\mu$ strain/inch (145-155 $\mu$ strain $/ \mathrm{mm}$ ) for GFRP. The rate of strain propagation can be related to the concrete strength, the stronger the concrete the lower the rate. This has been confirmed independently by a Japanese study [14].

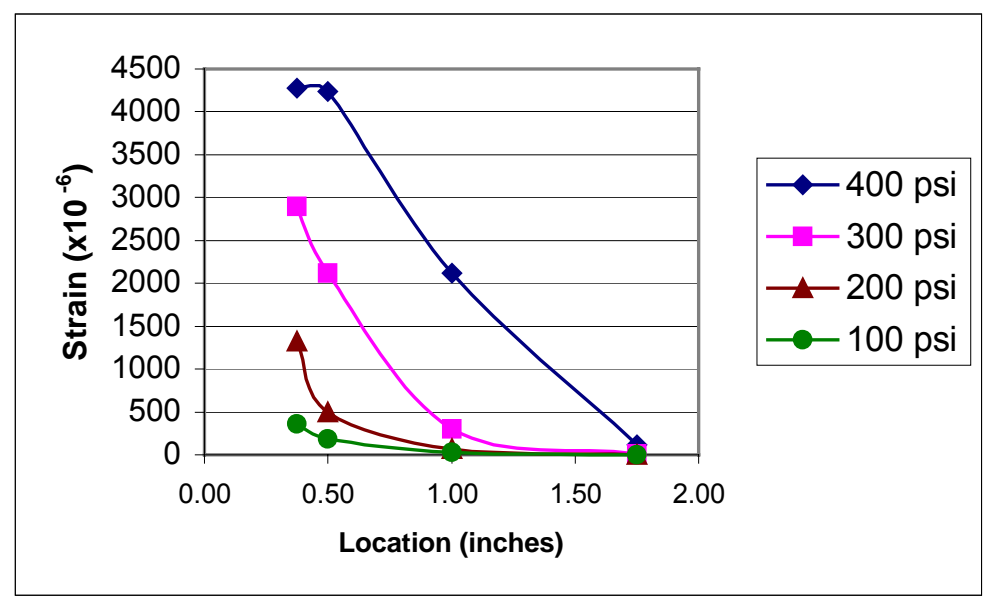

Figure 5.2 Strain as a Function of Location at Different Stress Levels for GFRP control 


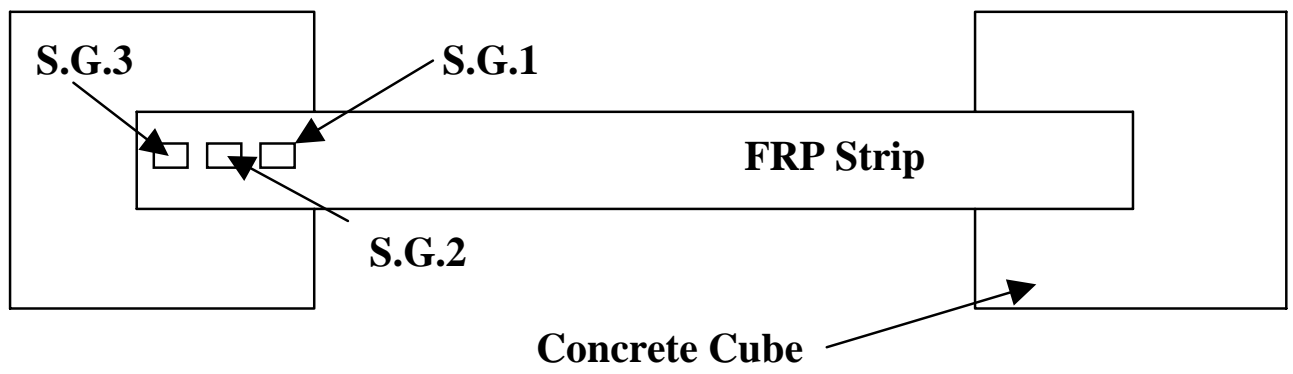

Figure 5.3 Gage location for GFRP Control \#1

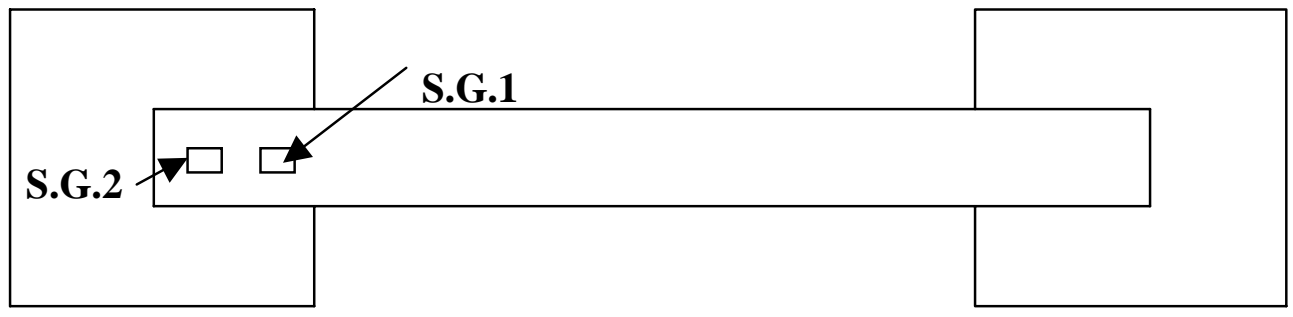

Figure 5.4 Gage location for GFRP Control \#2

Table 5.1 GFRP Control Stress and Strain Ratios

\begin{tabular}{|c|c|c|c|c|c|c|}
\hline $\begin{array}{l}\text { GFRP } \\
\text { Control }\end{array}$ & $\begin{array}{c}\text { A: Stress at } \\
\text { Bifurcation (psi) }\end{array}$ & $\begin{array}{l}\text { B: Stress at } \\
\text { Failure (psi) }\end{array}$ & $A / B$ & $\begin{array}{c}\text { C: Strain at } \\
\text { Bifurcation }(\mu \varepsilon)\end{array}$ & $\begin{array}{l}\text { D: Strain at } \\
\text { Failure }(\mu \varepsilon)\end{array}$ & $C / D$ \\
\hline Unaged & 180 & 380 & .47 & 600 & 3980 & 0.15 \\
\hline
\end{tabular}




\subsubsection{Carbon-Control}

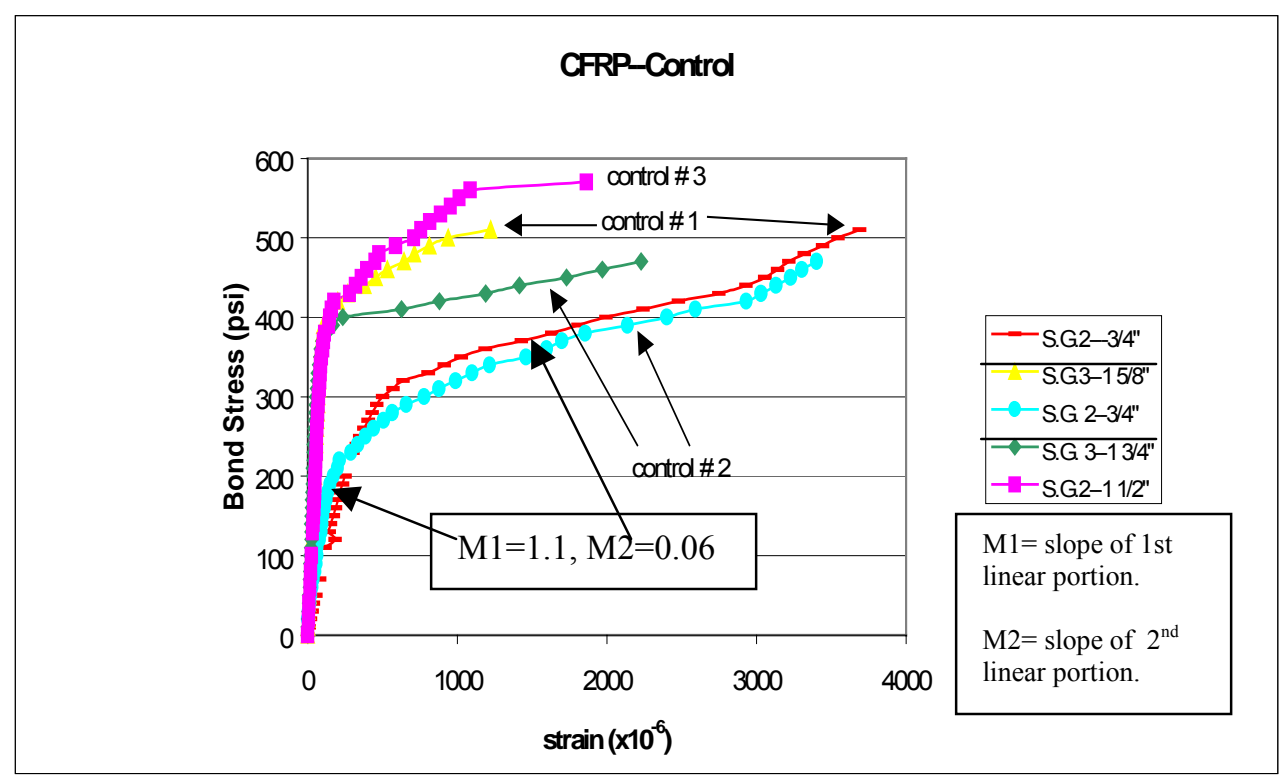

Figure 5.5 Strain Distribution for three Control Specimens with Carbon strips

The results in Figure 5.5 are from three different control specimens using carbon strips. The ultimate bond strength is 570 psi. The average bond strength, found from averaging the ultimate bond strength of all three specimens, is $517 \mathrm{psi}$. The bi-linear behavior in this strain distribution graph is very similar to that of the GFRP control specimens, as shown in Figure 5.1. 


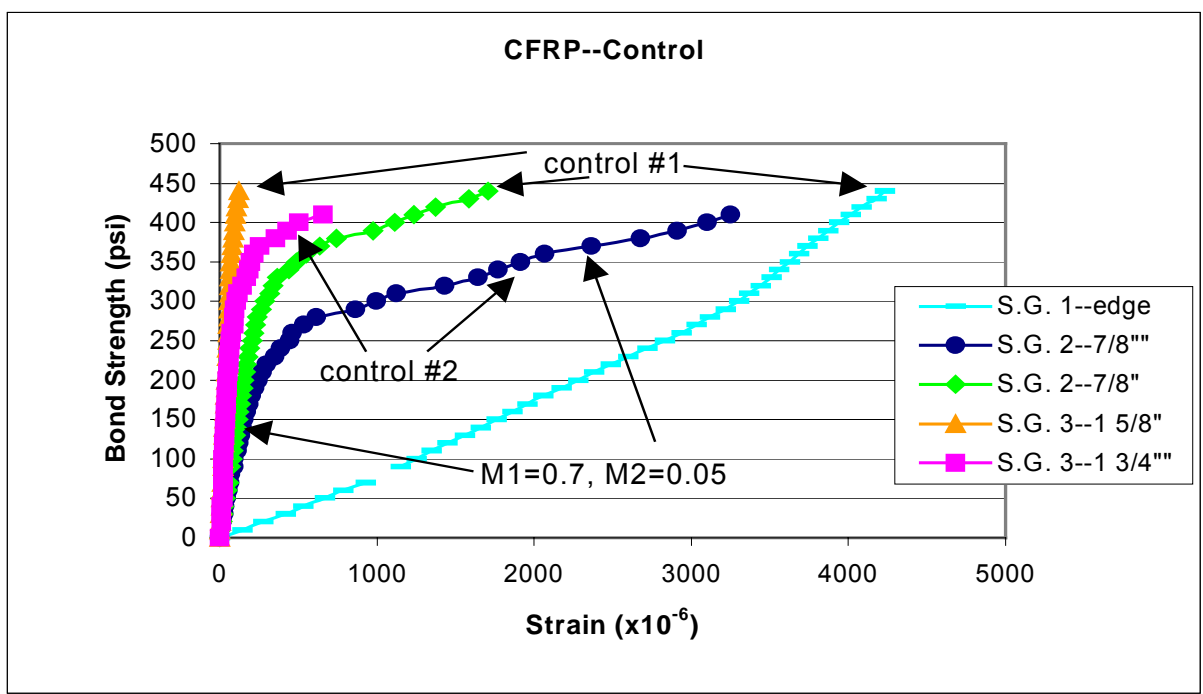

Figure 5.6 Strain Distribution for Control Specimens with Carbon Strips

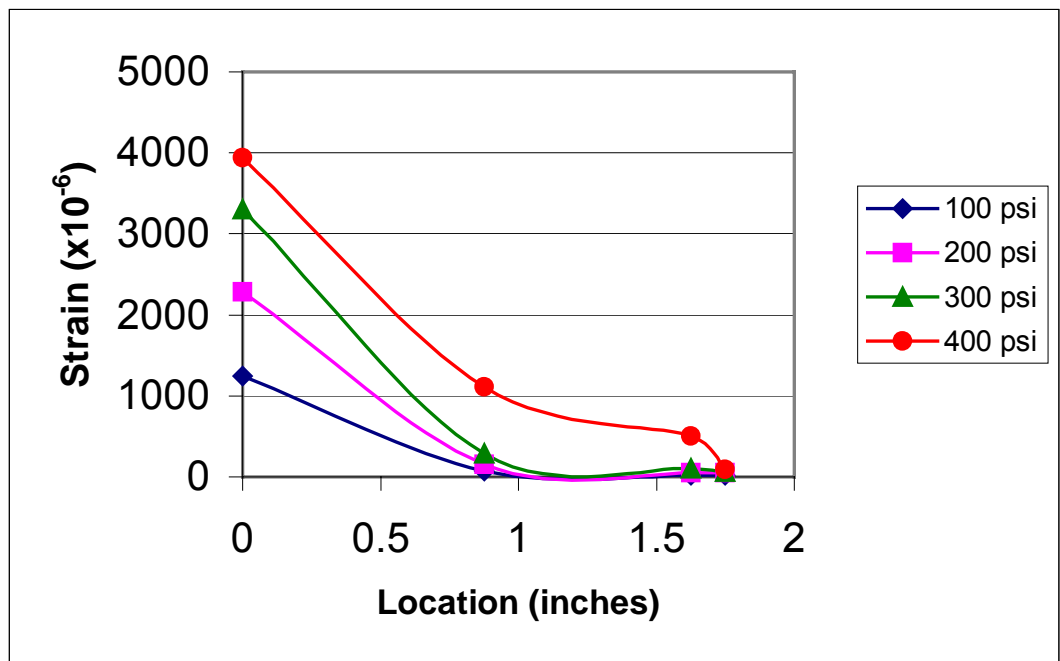

Figure 5.7 Strain Distribution as a Function of Location at Different Stress Levels

Figure 5.6 is another graph for a second set of control specimens using carbon strips. The gages listed in the legend are from two different unaged specimens that were superimposed to develop a single graph (similar to the previous case shown in Figure 5.5). Figure 5.7 shows the strain as a function of location for the specimens of Figure 5.6. The strain distribution rate for CFRP was calculated to be about $3302 \mu$ strain/inch 
(130 $\mu$ strain/mm). In a similar study, Maeda [14] found a rate of about 110-115

$\mu$ strain/mm for CFRP. The difference in our results from the Japanese results can be explained from the variation in strength of the concrete. In this study f'c is 4 ksi whereas in Maeda's study it was 6 ksi. A higher strength concrete would demonstrate less strain than a lower strength concrete, because of higher modulus of elasticity of concrete.

In Figure 5.6, the gage on the edge, S.G.1, measured composite stress in the unbonded part of the strip. It can be seen that the strain is decreasing as the bond length is increasing. This is due to the fact that bond stress is not constant over its overlapping length and it is distributed over a certain length in a parabolic form, and goes to zero beyond a certain distance from the edge $[2,6]$. It can also be observed that after the point of bifurcation the strain is increasing rapidly unto failure. Because in-plane bond strain concentration is the highest at the edge due to stress concentration near edges (or edge effects) and decreases with the increasing edge distance, sudden increase in strain is seen sooner in gages that are closest to the edge of the bond.

Table 5.2 CFRP Control Stress and Strain Ratios

\begin{tabular}{|l|c|c|c|c|c|c|}
\hline CFRP & $\begin{array}{c}\text { A: Stress at } \\
\text { Control }\end{array}$ & $\begin{array}{c}\text { B: Stress at } \\
\text { Fifurcation }(p s i)\end{array}$ & A / B & $\begin{array}{c}\text { C: Strain at } \\
\text { Bifurcation }(\mu \varepsilon)\end{array}$ & $\begin{array}{c}\text { D: Strain at } \\
\text { Failure }(\mu \varepsilon)\end{array}$ & $\begin{array}{c}\text { C / D } \\
\text { Unaged }\end{array}$ \\
\hline
\end{tabular}




\subsection{Conditioned Specimens}

In this section the bar charts show the comparison of bond strength of the conditioned specimens versus the unconditioned ones. The graphs depict the strain distribution for the aged specimens. GFRP results are given first followed by CFRP in section 5.3.2.

\subsubsection{Glass--GFRP}

The following sections (5.3.1.1 $-5.3 .1 .5)$ provide results for accelerated aged glass specimens.

\subsubsection{Acid and Alkaline-Room Temperature}

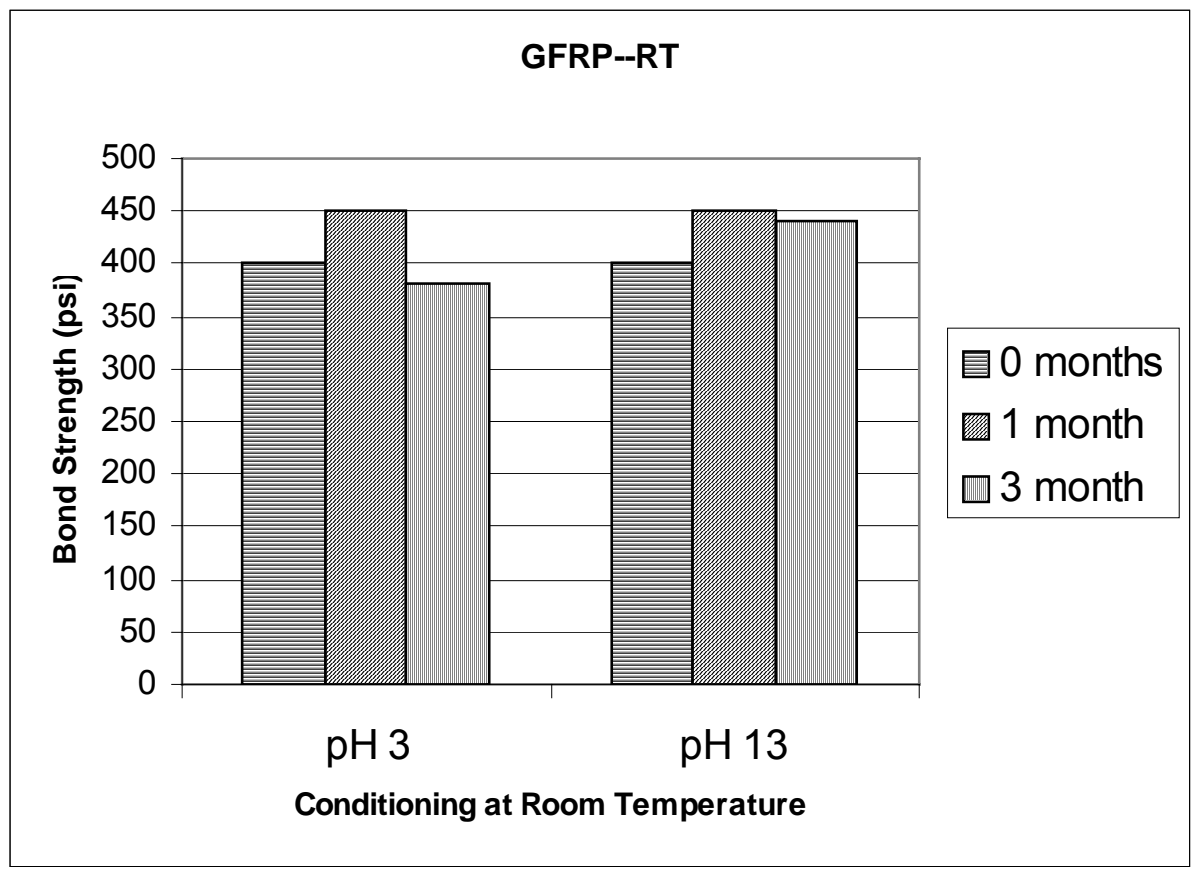

Figure 5.8 Effects of Aging on Bond Strength Between GFRP and Concrete 
Figure 5.8 gives the bond strength for specimens that were aged at room temperature while soaking in the solutions. In test samples conditioned for both the acid and salt, the bond strength initially increases due to better curing of resin and then begins to decrease as the resin is aged due to plasticization. The acid environment seems to have more adverse effect on bond strength than the alkaline environment.

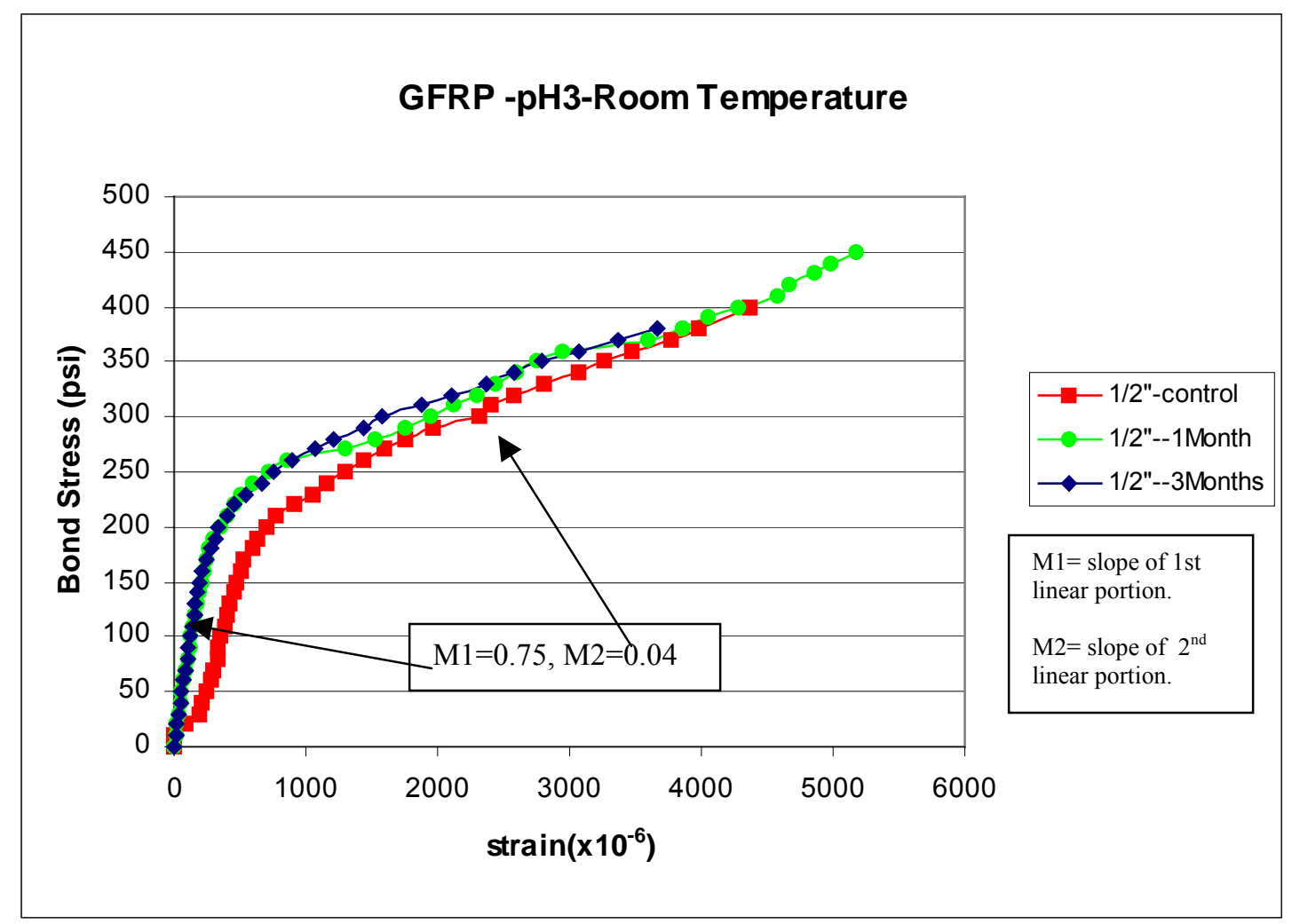

Figure 5.9 Strain Distribution of GFRP/pH3/RT

It can be seen in figure 5.9 that the strain distribution is very similar for all three aging durations. If the first few points from the control line were to be removed then all three lines would follow the same path, thus suggesting that no bond strength was lost upon aging. It can also be seen that the lines make a change in slope when the stress level is between $200-250 \mathrm{psi}$ and the strain is about 400 microstrains. The chart also reveals that the specimens fail at a different strain and stress level. 
Figure 5.10 gives the strain distribution for a GFRP specimen soaking in an alkaline solution. It was discovered that almost all glass specimens that were exposed to alkaline conditions had a premature failure within the strip itself. However, due to specimen replications, more than one specimen was available from this aging condition. Therefore, just prior to testing, unaged strips were bonded onto the aged strips so as to prevent fabric failure. This was done in such a manner as to keep the sample undisturbed in terms of aging of bond between the first layer and the concrete surface.

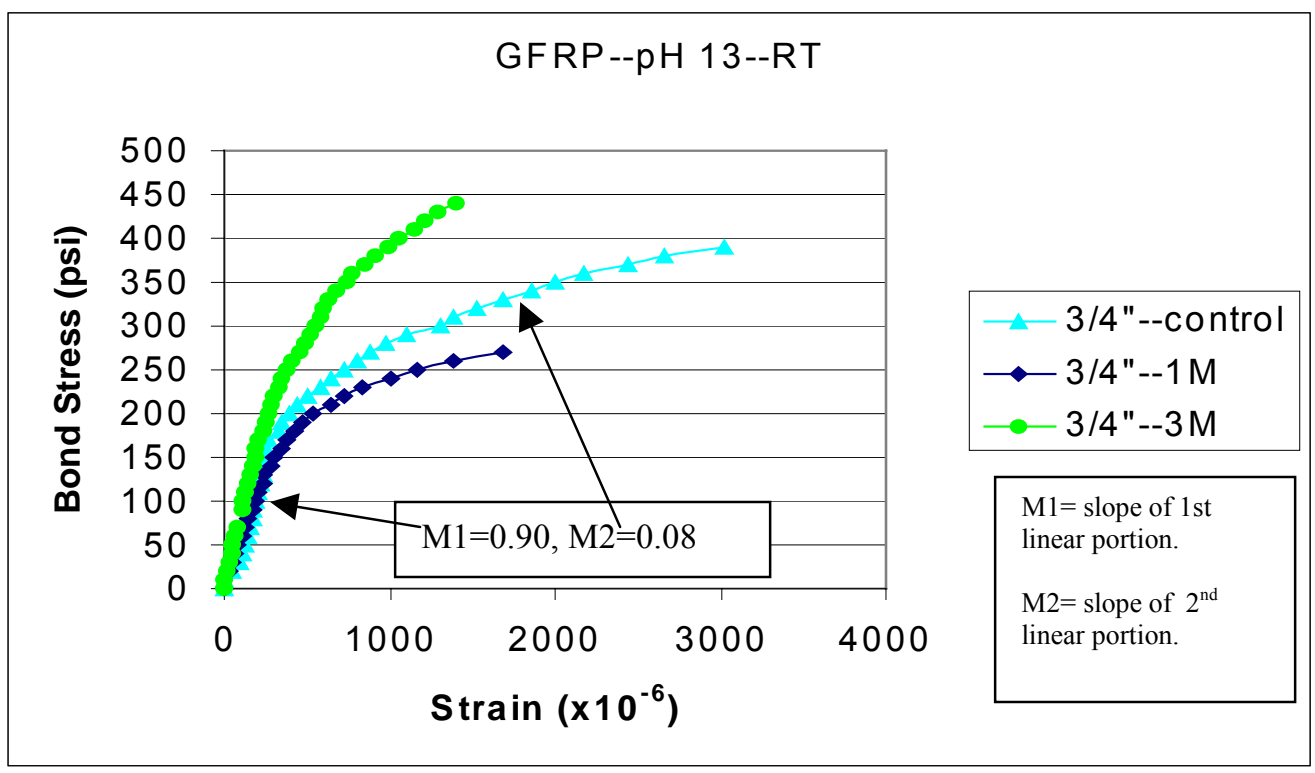

Figure 5.10 Strain Distribution for GFRP/pH 13/RT

In figure 5.10 the specimens were aged at room temperature. Because the gages were located on the second layer (reinforcing layer to prevent fiber failure) rather than the first, the strain readings may be lower than those recorded for the single layered specimens, i.e. 100\% compositeness (shear transfer) between layers of GFRP strips may 
not have developed from the glue line or bond resistance from resin may have deteriorated with aging.

Table 5.3 GFRP/pH 13/RT Stress and Strain Ratios

\begin{tabular}{|l|c|c|c|c|c|c|}
\hline GFRP-pH 13 & $\begin{array}{c}\text { A: Stress at } \\
\text { Room Temp. }\end{array}$ & $\begin{array}{c}\text { B: Stress at } \\
\text { Bifurcation (psi) }\end{array}$ & Failure (psi) & A / B & $\begin{array}{c}\text { C: Strain at } \\
\text { Bifurcation }(\mu \varepsilon)\end{array}$ & $\begin{array}{c}\text { D: Strain at } \\
\text { Failure }(\mu \varepsilon)\end{array}$ \\
\hline 1 month & 200 & 450 & .44 & 300 & 5100 & 0.06 \\
\hline 3 months & 200 & 390 & .51 & 300 & 3750 & 0.08 \\
\hline
\end{tabular}

Table 5.4 GFRP/pH 3/RT Stress and Strain Ratios

\begin{tabular}{|l|c|c|c|c|c|c|}
\hline GFRP-pH 3 & $\begin{array}{c}\text { A: Stress at } \\
\text { Room Temp. }\end{array}$ & $\begin{array}{c}\text { B: Stress at } \\
\text { Bifurcation (psi) }\end{array}$ & Failure (psi) & A / B & $\begin{array}{c}\text { C: Strain at } \\
\text { Bifurcation }(\mu \varepsilon)\end{array}$ & $\begin{array}{c}\text { D: Strain at } \\
\text { Failure }(\mu \varepsilon)\end{array}$ \\
\hline 1 month & 150 & 270 & .55 & 250 & 1740 & 0.14 \\
\hline 3 months & 225 & 440 & .51 & 250 & 1375 & 0.18 \\
\hline
\end{tabular}

Tables 5.3 and 5.4 give the ratios of the stress and strain at the point of bifurcation to the value at failure. The bifurcation takes place at a range of $45 \%-55 \%$ of ultimate load. 


\subsubsection{Acid and Alkaline—Freeze-thaw}

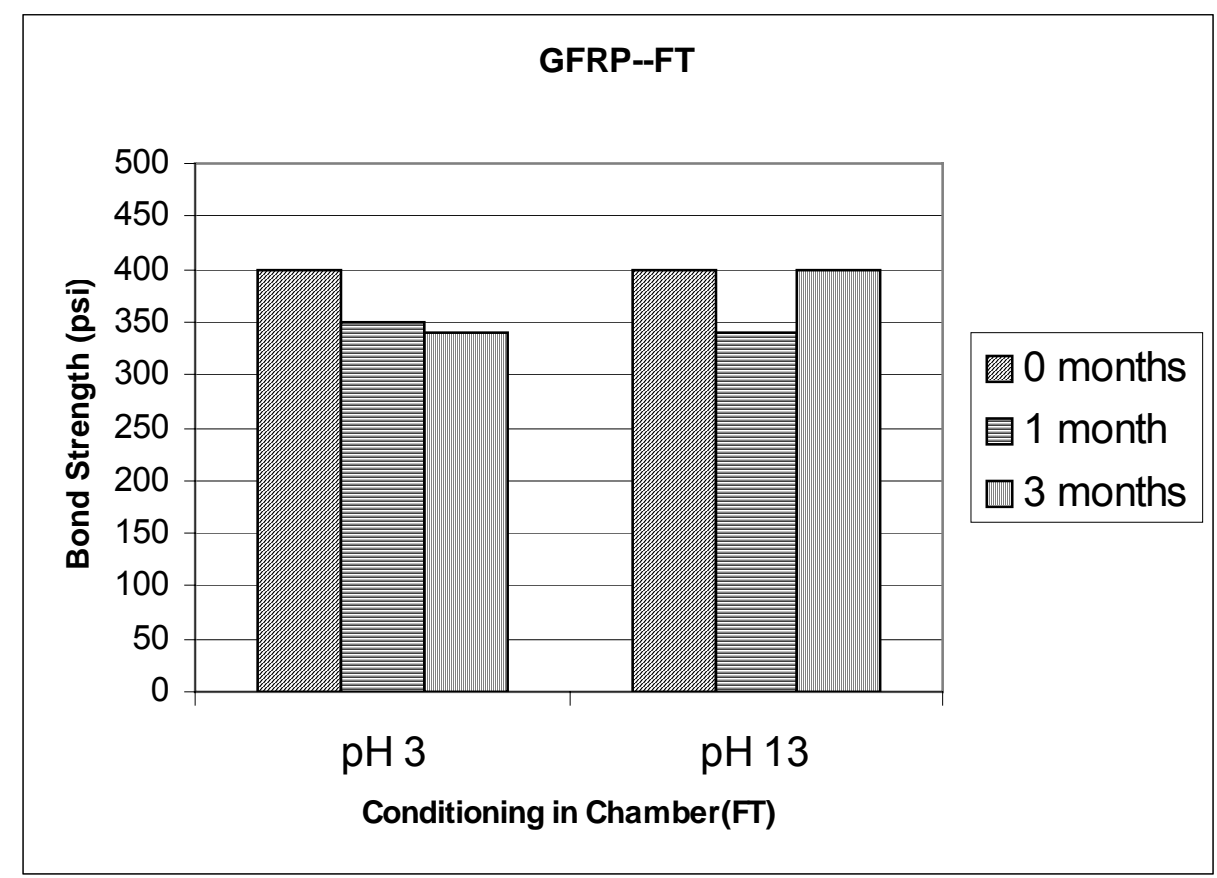

Figure 5.11 Effects of Aging on Bond Strength Between GFRP and Concrete

Figure 5.11 gives the results for glass specimens soaked in $\mathrm{pH} 3$ and 13 solutions under freeze-thaw conditions. Acid shows a 3\% decrease in strength from 1 month to 3 months whereas alkaline solution based aging does not. However, when these results are compared to those aged samples at room temperature (Figure 5.8), it can be seen that the bond strength is decreasing $16 \%$ more with respect to freeze-thaw for both acid and alkaline conditions. This can be expected. Due to the thermal fluctuations, the freezethaw environment tends to be a more severe environment on bond strength than at room temperature. 


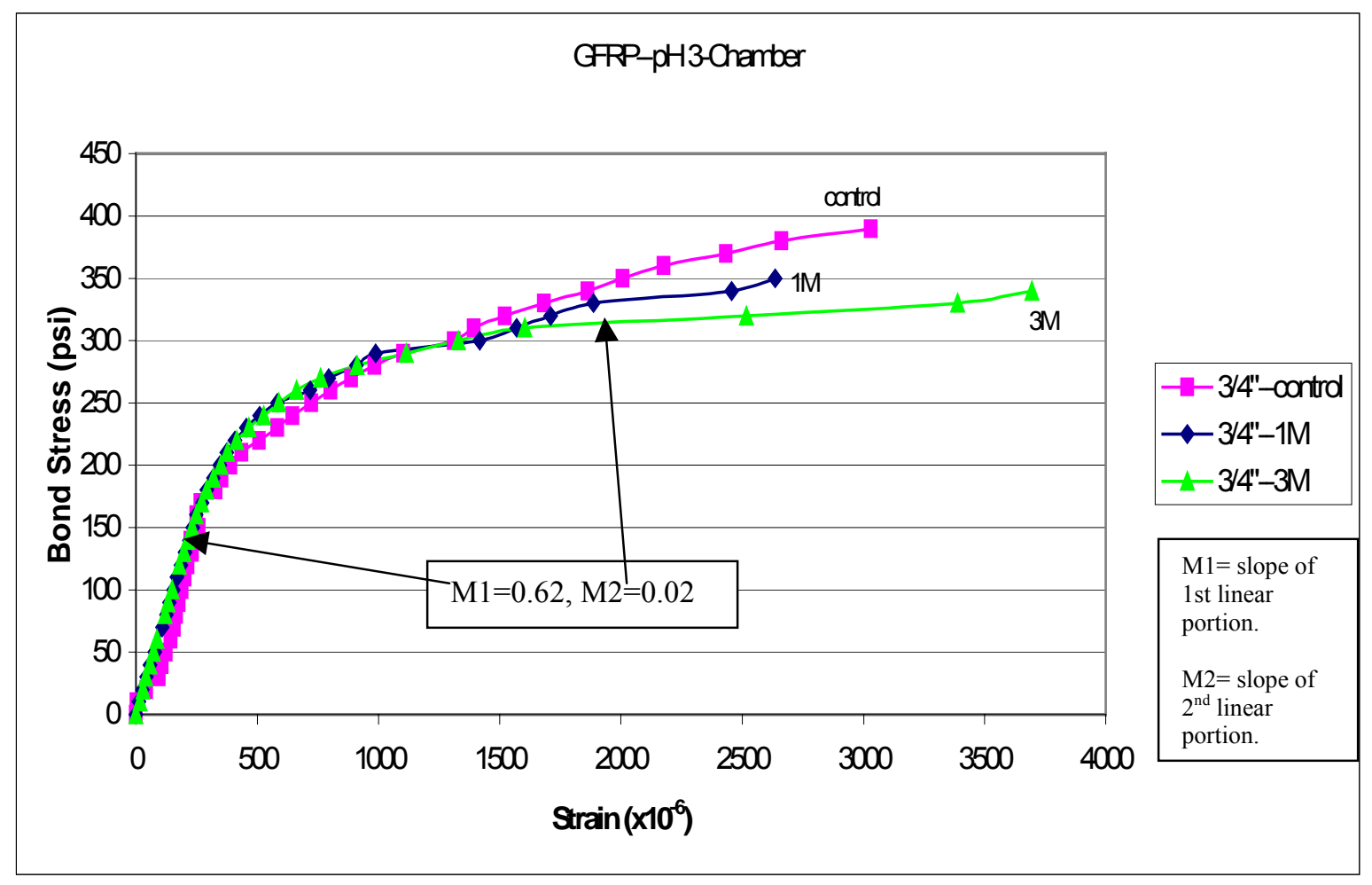

Figure 5.12 Strain Distribution for GFRP/pH3/chamber(FT)

Figure 5.12 follows much of the same characteristics as the previous ones. The linear portion of the lines can be attributed to the properties of fibers, resin and the concrete strength. At the second linear portion of the lines, the slope is thought to be related mostly to the resin properties. This is because during initial loading the resin, glass and concrete elongate together; but at approximately 300 microstrains concrete begins to fracture. The resin compensates for the loss of the concrete strength by trying to carry the load. Then, plasticization of adhesive leads to greater strain rate with respect to stress. However, concrete deterioration is what is thought to lead to failure. 


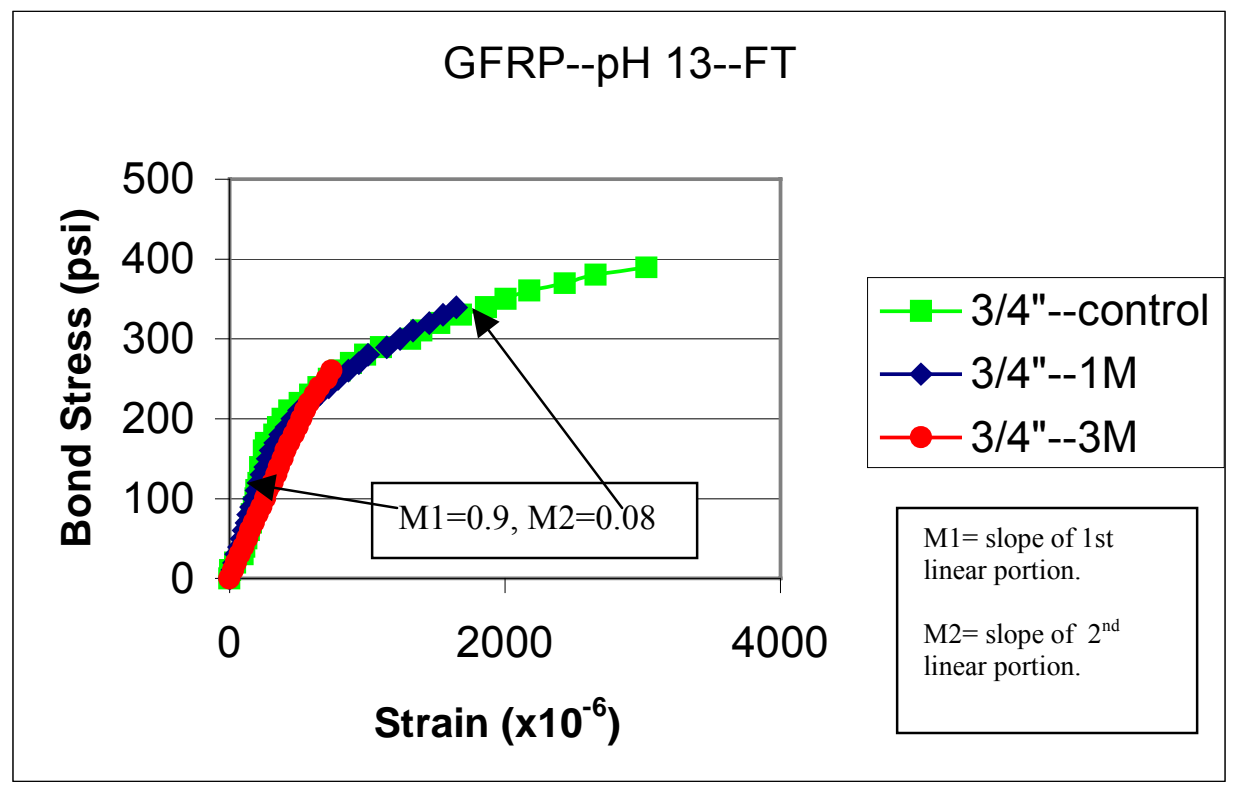

Figure 5.13 Strain Distribution for GFRP/pH 13/FT

Figure 5.13 is the strain distribution for glass specimens soaking in an alkaline solution in the environmental chamber. It reveals that the aged specimens tend to be following the same distribution as the control specimens. As in the previous case involving alkaline and glass (Figure 5.10), the specimens required an additional layer of glass fiber reinforcement to prevent premature failure in the fabric. Because of this, the low strains are the result of having the strain gages on the second layer.

When Figures 5.13 and 5.10 are compared, it can be seen that the slopes (M1 \& M2) are identical. Therefore, maybe the freeze-thaw environment is no more detrimental to the strain distribution than is room temperature. 
Table 5.5 GFRP/pH 3/FT Stress and Strain Ratios

\begin{tabular}{|l|c|c|c|c|c|c|}
\hline GFRP-pH 3 & $\begin{array}{c}\text { A: Stress at } \\
\text { Room Temp. }\end{array}$ & $\begin{array}{c}\text { B: Stress at } \\
\text { Bifurcation (psi) }\end{array}$ & Failure (psi) & A / B & $\begin{array}{c}\text { C: } \text { Strain at } \\
\text { Bifurcation }(\mu \varepsilon)\end{array}$ & $\begin{array}{c}\text { D: Strain at } \\
\text { Failure }(\mu \varepsilon)\end{array}$ \\
\hline 1 month & 200 & 350 & .57 & 300 & 2625 & 0.11 \\
\hline 3 months & 200 & 340 & .58 & 300 & 3710 & 0.08 \\
\hline
\end{tabular}

Table 5.6 GFRP/pH 13/FT Stress and Strain Ratios

\begin{tabular}{|l|c|c|c|c|c|c|}
\hline GFRP-pH 13 & $\begin{array}{c}\text { A: Stress at } \\
\text { Room Temp. }\end{array}$ & $\begin{array}{c}\text { B: Stress at } \\
\text { Bifurcation (psi) }\end{array}$ & Failure (psi) & A B & $\begin{array}{c}\text { C: Strain at } \\
\text { Bifurcation }(\mu \varepsilon)\end{array}$ & $\begin{array}{c}\text { D: Strain at } \\
\text { Failure }(\mu \varepsilon)\end{array}$ \\
\hline 1 month & 200 & 340 & .58 & 490 & 1710 & 0.29 \\
\hline 3 months & 200 & 260 & .77 & 490 & 814 & 0.60 \\
\hline
\end{tabular}

Tables 5.5 and 5.6 give the ratios of stress versus strain at the points of bifurcation to the values at failure. Again, the low strain values at failure can be directly related to the additional reinforcement that was required to prevent premature failure in glass fibers exposed to $\mathrm{pH} 13$ solution. The low failure stress of the three month aged specimen in table 5.6 may be the result of a bad specimen sample, i.e., misalignment of strips or rough concrete surface. 


\subsubsection{Freeze-thaw (FT)}

The specimens (Figure 5.14) were placed in the environmental chamber to undergo freeze-thaw conditioning. They were not immersed in any type of solution. However, some of these specimens were subjected to sustained stress ( $25 \%$ ult. bond strength) while others had no sustained stress.

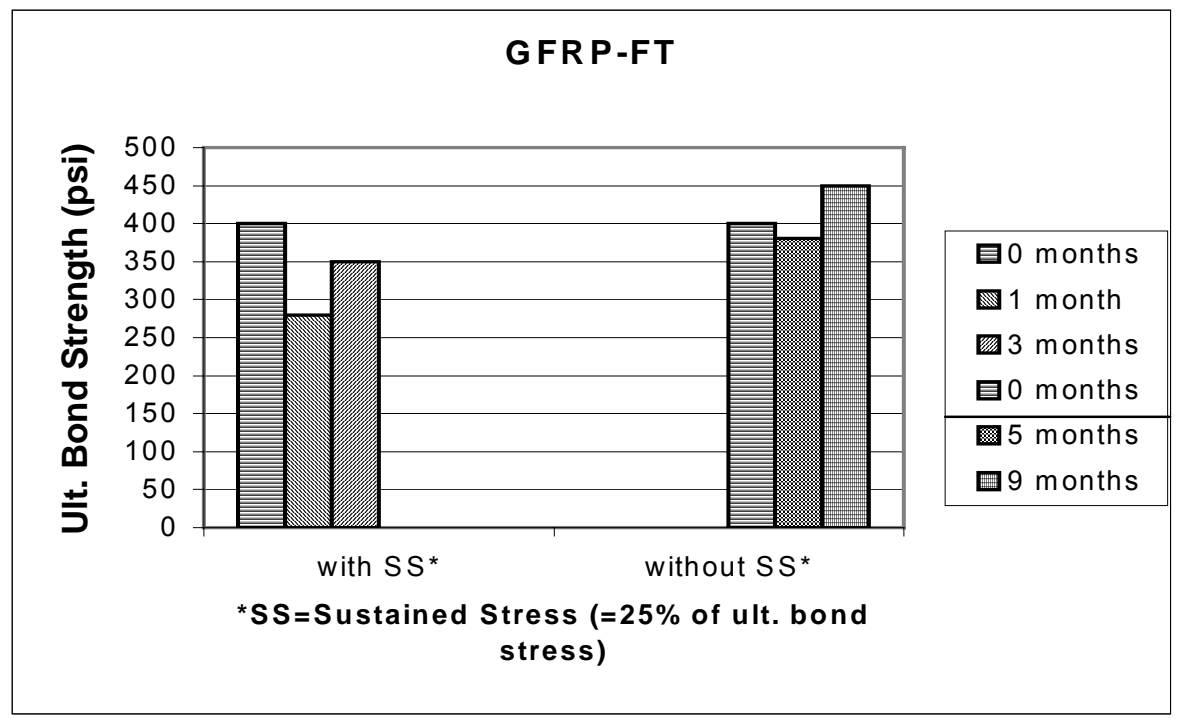

\section{Figure 5.14 Effects of Aging on Bond Strength Between GFRP and Concrete}

It can also be noted that the bond strength for specimens under sustained stress decreased by about $25 \%$ more than the specimens aged without any sustained stress. In both cases, the trends in bond strength results are the same, i.e., a decrease in strength followed by an increase. The decrease seen by the one month aged specimen under sustained stress may be explained from Horiguchi's research [4]. The study focused on the effect of test methods and quality of concrete on the bond strength of FRP sheets. Horiguchi suggested that a high correlation exists between the bond strength and the 
compressive strength of concrete. When the compressive strength was low, micro fracture occurred in the matrix of the concrete. Therefore, based on the method for curing used in this study, it is possible that some cubes were better cared for during curing than others. When some cubes were placed for curing, they were stacked upon each other. Wet burlap was placed over the cubes followed by a sheet of plastic to hold in the moisture. The cubes on the bottom of the stack did not receive much moisture and therefore may not have as high a compressive strength as the ones towards the top.

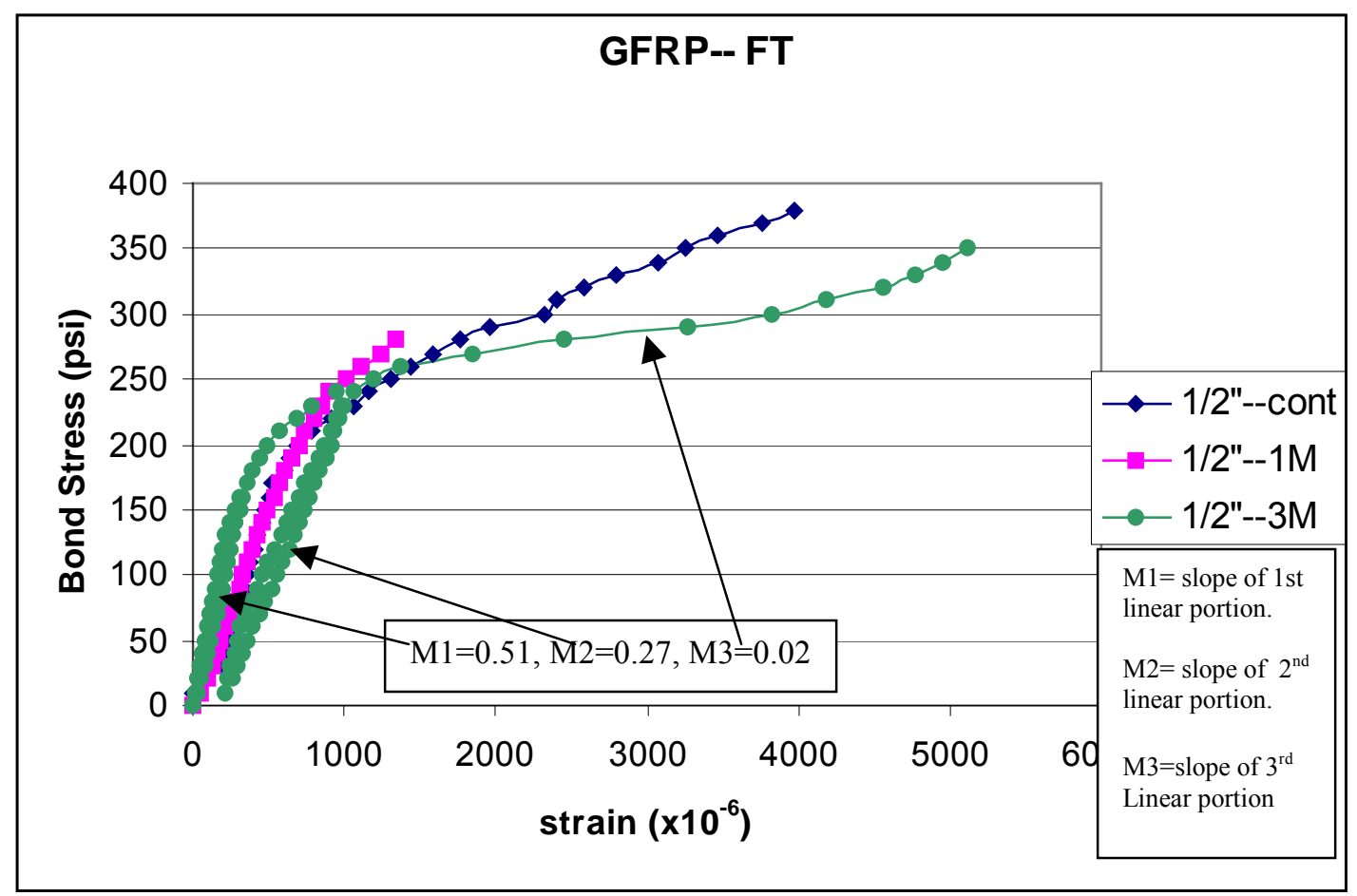

Figure 5.15 Strain Distribution for GFRP/FT under Loading-Unloading

Loading-Unloading Effects:

Figure 5.15 reveals again that the strain distribution is unchanged after aging. When the aged specimens were tested it was decided to load them to certain stress levels and then drop back to zero to see if the strains would be recovered. The specimens were 
stressed to levels of $80 \mathrm{psi}, 160 \mathrm{psi}$, and $240 \mathrm{psi}$. After returning to zero from the $240 \mathrm{psi}$ level, the specimen was tested to failure. In all the test specimens that this procedure was repeated, the 80 psi level was always recovered and in most cases so was the $160 \mathrm{psi}$ level. However, specimens tested to 240 psi level did not usually recover back to zero strain. This was done to see when and where the adhesive was changing its characteristics. Again, the low strain value to failure of the one month aged specimen can be attributed to poor concrete.

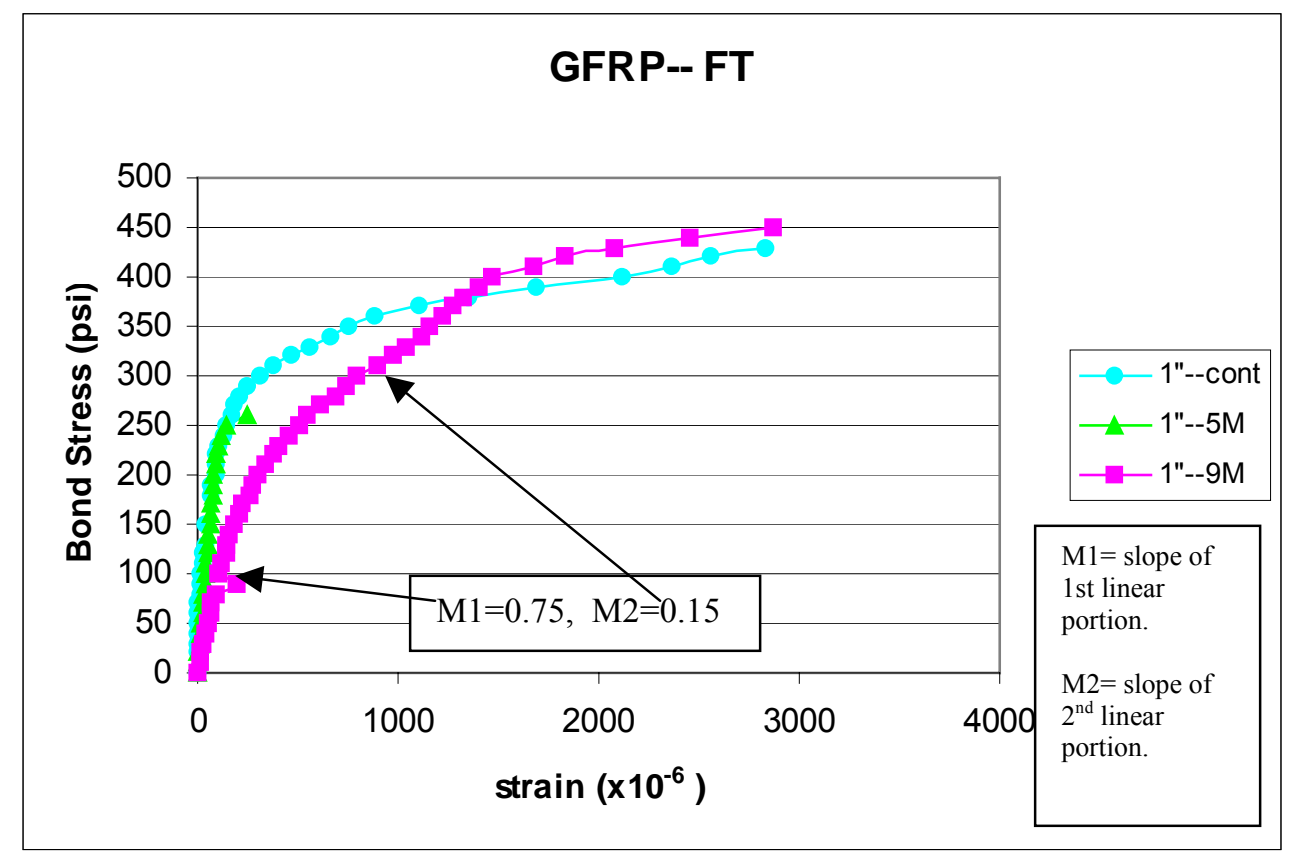

Figure 5.16 Strain Distribution for GFRP/FT

Figure 5.16 gives results for specimens aged for 5 and 9 months along with the results for the control specimen. These strain readings were recorded one inch back from the beginning of the bond area. These results are revealing the same distribution as in all the previous cases. Because strain readings at the $1 / 2$ " location were unavailable due to a faulty gage, it should be noted that the strain readings were taken at a distance farther 
back from the edge of the concrete cube along the strip than in the previous cases (1" vs. $1 / 2$ " or $3 / 4 ")$. Therefore, the nonlinearity begins about 50 psi higher stress than in the previous cases. However, due to plasticization, nonlinearity of the nine month aged specimen still occurred at about the same stress levels as those of the preceding test specimens. The strain at the change in slope seems to remains constant no matter the gage location. The low failure strain of the 5 month specimen can again be related to poor concrete quality and/or placement (alignment) of strips.

Table 5.7 GFRP/FT Stress and Strain Ratios

\begin{tabular}{|l|c|c|c|c|c|c|}
\hline GFRP--FT & $\begin{array}{c}\text { A: Stress at } \\
\text { Bifurcation (psi) }\end{array}$ & $\begin{array}{c}\text { B: Stress at } \\
\text { Failure (psi) }\end{array}$ & A / B & $\begin{array}{c}\text { C: Strain at } \\
\text { Bifurcation }(\mu \varepsilon)\end{array}$ & $\begin{array}{c}\text { D: Strain at } \\
\text { Failure }(\mu \varepsilon)\end{array}$ & $\begin{array}{c}\text { C / D } \\
\end{array}$ \\
\hline 1 month & 200 & 280 & .71 & 600 & 1400 & 0.43 \\
\hline 3 months & 170 & 350 & .49 & 450 & 5100 & 0.09 \\
\hline
\end{tabular}

Table 5.8 GFRP/FT Stress and Strain Ratios

\begin{tabular}{|c|c|c|c|c|c|c|}
\hline GFRP--FT & $\begin{array}{c}\text { A: Stress at } \\
\text { Bifurcation (psi) }\end{array}$ & $\begin{array}{l}\text { B: Stress at } \\
\text { Failure (psi) }\end{array}$ & $A / B$ & $\begin{array}{c}\text { C: Strain at } \\
\text { Bifurcation }(\mu \varepsilon)\end{array}$ & $\begin{array}{l}\text { D: Strain at } \\
\text { Failure }(\mu \varepsilon)\end{array}$ & $C / D$ \\
\hline 9 months & 200 & 450 & .44 & 300 & 2852 & 0.11 \\
\hline
\end{tabular}

Tables 5.7 and 5.8 give the ratios of the stress and strain at the point of bifurcation to the stress and strain values at failure. The nine month specimen was aged without a sustained stress. The stress and strain at bifurcation is about $50 \%$ and $10 \%$ of the failure values respectively. With the exception of the one month specimen, the values are falling 
into the same range as those from the previous aging conditions. In comparing the specimens, the failure stress is increasing along with bifurcation strain. This is due to plasticization. Strain to failure goes up from 1 month to 3 months and starts decreasing at the 9 month level.

\subsubsection{Water Conditioning}

The following results are from specimens that were placed in water at room

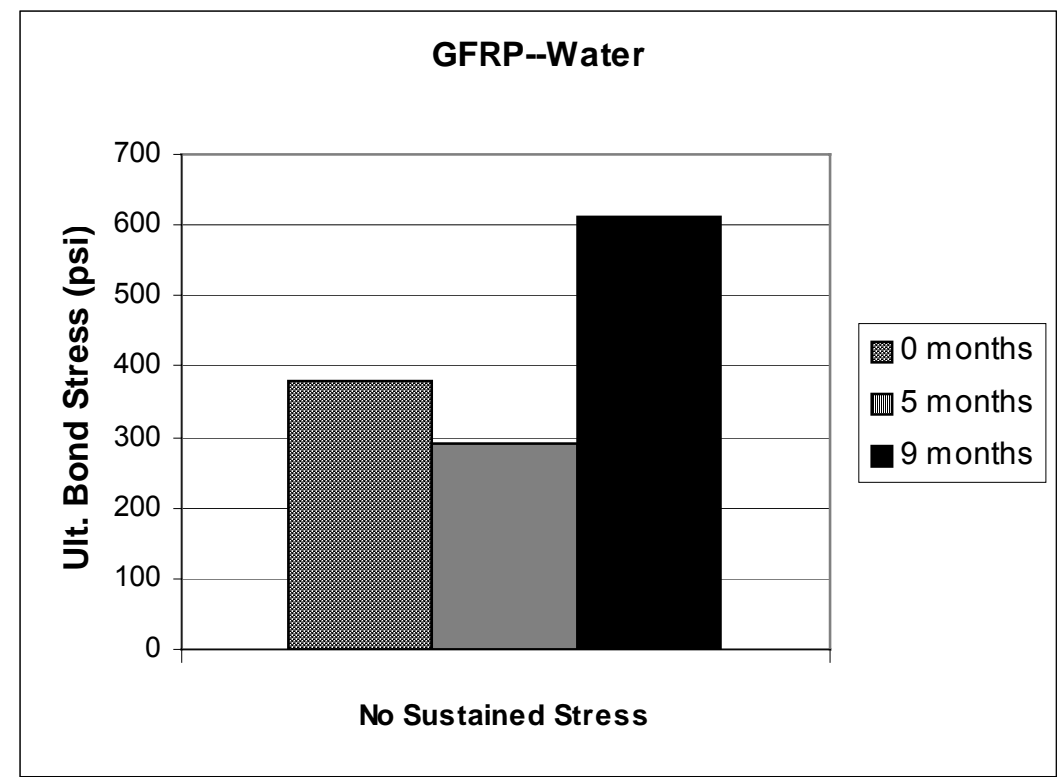

temperature.

\section{Figure 5.17 Effects of Moisture on Bond Strength between GFRP and Concrete}

Figure 5.17 shows a decrease in strength followed by an increase. The reasons for this may be due to a longer curing time for the nine month specimen. Of all the specimens tested in this study, this nine month specimen soaked in water demonstrated 
the highest bond strength. This may be due to improved cross-linking of chemical bonds [7] with time.

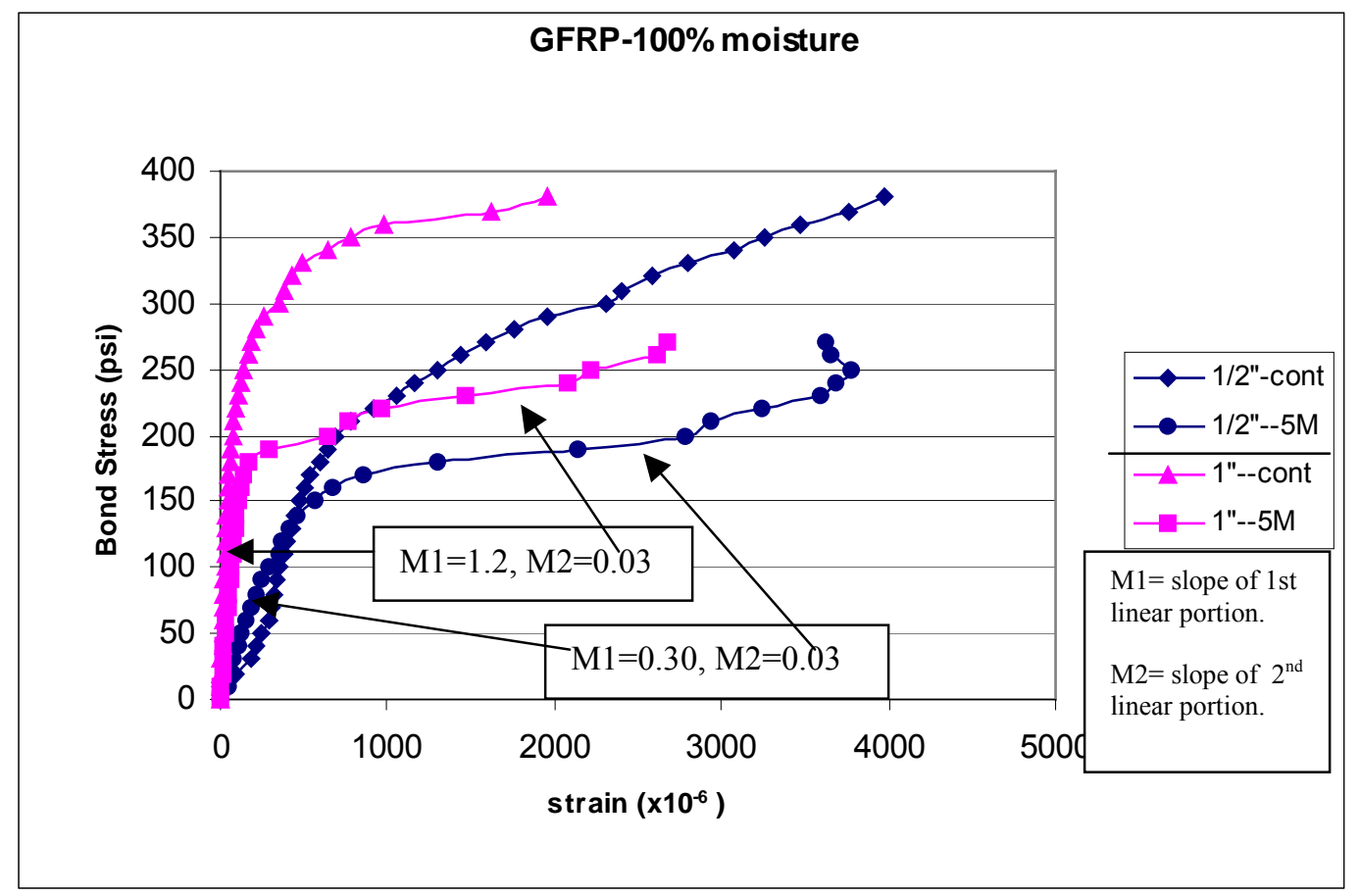

Figure 5.18 Strain Distribution of GFRP/water

Figure 5.18 provides results of glass specimen soaked in water for five months. The strain distribution is given at $1 / 2 "$ and $1 "$ distances from the edge of the cube, i.e., where bond stress started to resist. The results here seem to reveal that the distribution was not altered upon soaking. Even though the slope change occurs at a lower stress level than that of the unaged, the slope remains unchanged. The slope change may suggest that as the moisture is being absorbed, the adhesive is plasticizing. Therefore, we conclude that increase in moisture absorption leads to increase in plasticization. As it can be seen, slope of the aged specimen decreases, suggesting that more plasticizing has occurred. This may be what is occurring in all specimens that are being exposed to 
moisture. The severity of reduction is represented by the slope change of the line after plasticizing begins.

Table 5.9 GFRP/Water Stress and Strain Ratios

\begin{tabular}{|l|c|c|c|c|c|c|}
\hline GFRP-- & $\begin{array}{c}\text { A: Stress at } \\
\text { WATER }\end{array}$ & $\begin{array}{c}\text { B: Stress at } \\
\text { Bifurcation (psi) }\end{array}$ & A / B & $\begin{array}{c}\text { C: Strain at } \\
\text { Bifurcation }(\mu \varepsilon)\end{array}$ & $\begin{array}{c}\text { D: Strain at } \\
\text { Failure }(\mu \varepsilon)\end{array}$ & C / D \\
\hline $\begin{array}{l}5 \text { month } \\
@ 1 / 2 \text { " gage }\end{array}$ & 150 & 270 & .55 & 500 & 3600 & 0.14 \\
\hline $\begin{array}{l}5 \text { months @ } \\
1 \text { "gage }\end{array}$ & 170 & 270 & .62 & 250 & 2700 & 0.09 \\
\hline
\end{tabular}

Table 5.9 gives the ratios of the stress and strain at the point of bifurcation to the stress and strain values at failure. The stress and strain at bifurcation is about $55 \%$ and $10 \%$ of the failure values respectively. The values here agree with the values from the previous conditions. 


\subsubsection{Natural Aging}

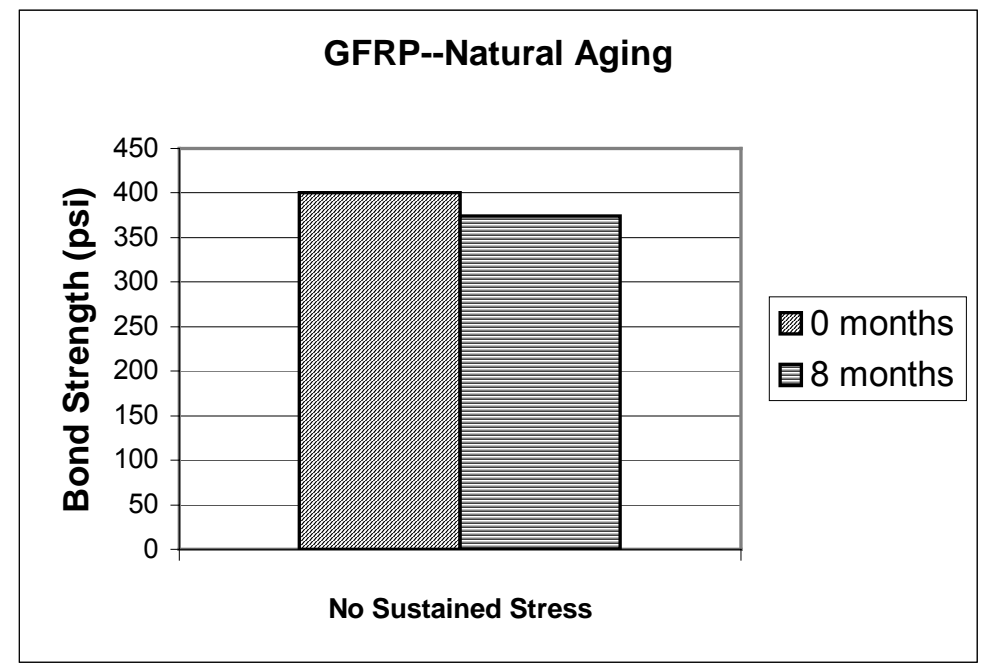

Figure 5.19 Bond Strength of Naturally Aged GFRP Specimens

In Figure 5.19, bond strength is given for glass specimens that were placed outside for eight months of conditioning. The bond strength of $360 \mathrm{psi}$ for the naturally aged specimens was determined by averaging the ultimate strength of all the naturally aged tested specimens. The strength is within $6 \%$ of the unaged suggesting that no severe degradation is evident. These specimens were placed outside in January and remained there until summer was over, thus being exposed initially to cold weather and finally to warm weather. 


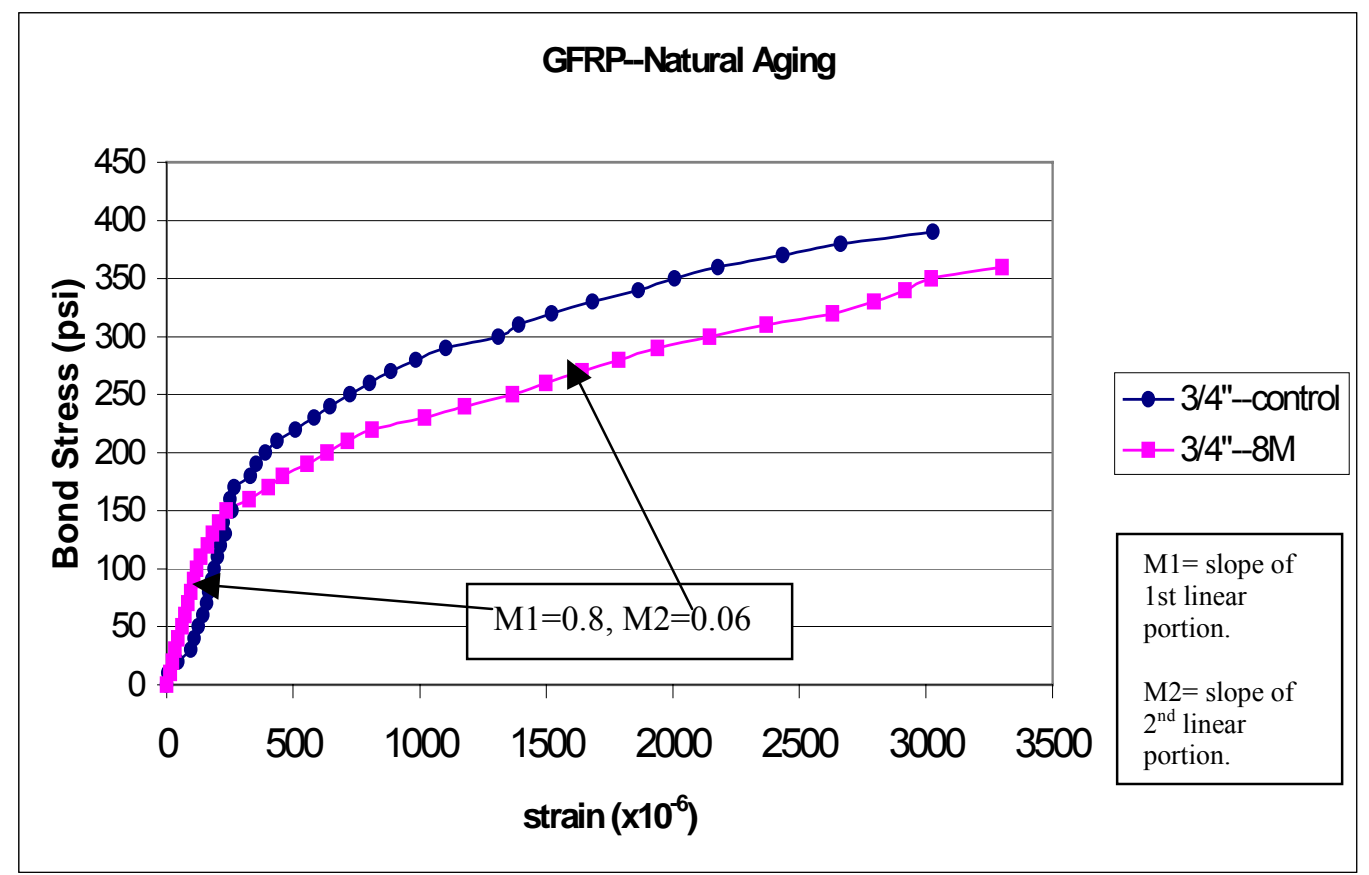

Figure 5.20 Strain Distribution for GFRP/Natural Aging

In figure 5.20 we see that the natural aging has caused the adhesive to plasticize a little. However, the distribution of strain across the bond area continues to be similar to that of the control specimens. It also appears that the difference in accelerated aging to that of natural aging is not significant with respect to bond degradation.

Table 5.10 GFRP/Natural Aging Stress and Strain Ratios

\begin{tabular}{|l|c|c|c|c|c|c|}
\hline GFRP- & $\begin{array}{c}\text { A: Stress at } \\
\text { Natural }\end{array}$ & B: Stress at & A / B & C: Strain at & D: Strain at & C / D \\
Aging & Bifurcation (psi) & Failure (psi) & & Bifurcation $(\mu \varepsilon)$ & Failure $(\mu \varepsilon)$ \\
\hline 8 month & 170 & 360 & .47 & 350 & 3320 & 0.11 \\
\hline
\end{tabular}

The ratio values in table 5.10 again fall in the range of 50\% stress and $10 \%$ strain. 


\subsubsection{Carbon--CFRP}

\subsubsection{Acid and Alkaline-Room Temperature}

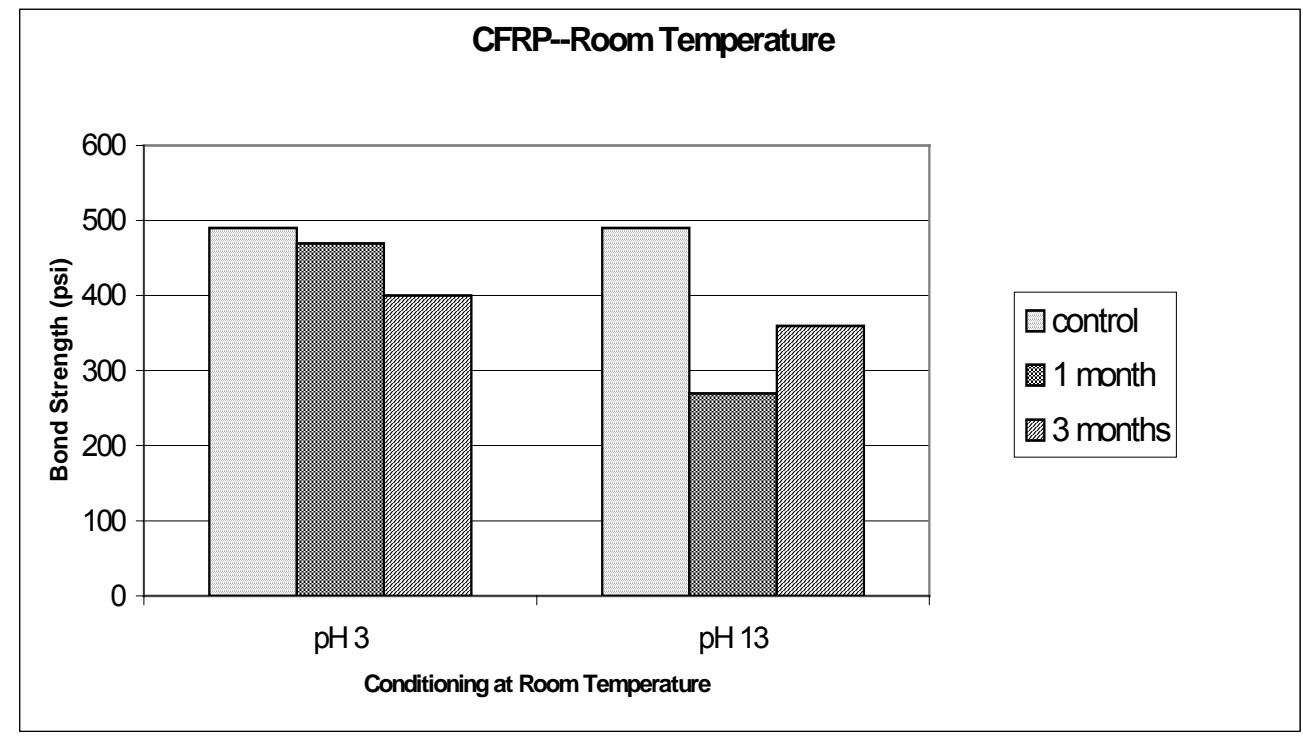

Figure 5.21 Effects of Aging on Bond Strength Between CFRP and Concrete

Figure 5.21 gives the results for carbon specimens soaking in solutions at room temperature. The acid shows a $15 \%$ decrease in bond strength with time, whereas, immersion in alkaline solution has an increase from one month to three months. This increase however is still well below the control specimen strength. The decrease in bond strength of the one month aged specimen immersed in alkaline may be due to improper application of epoxy in the bond area upon applying the strips or a reaction with the epoxy resulting in a loss of strength [7]. In contrast to glass (Figure 5.8), the alkaline solution seems to have a greater effect on bond strength of carbon strips than the acid. 


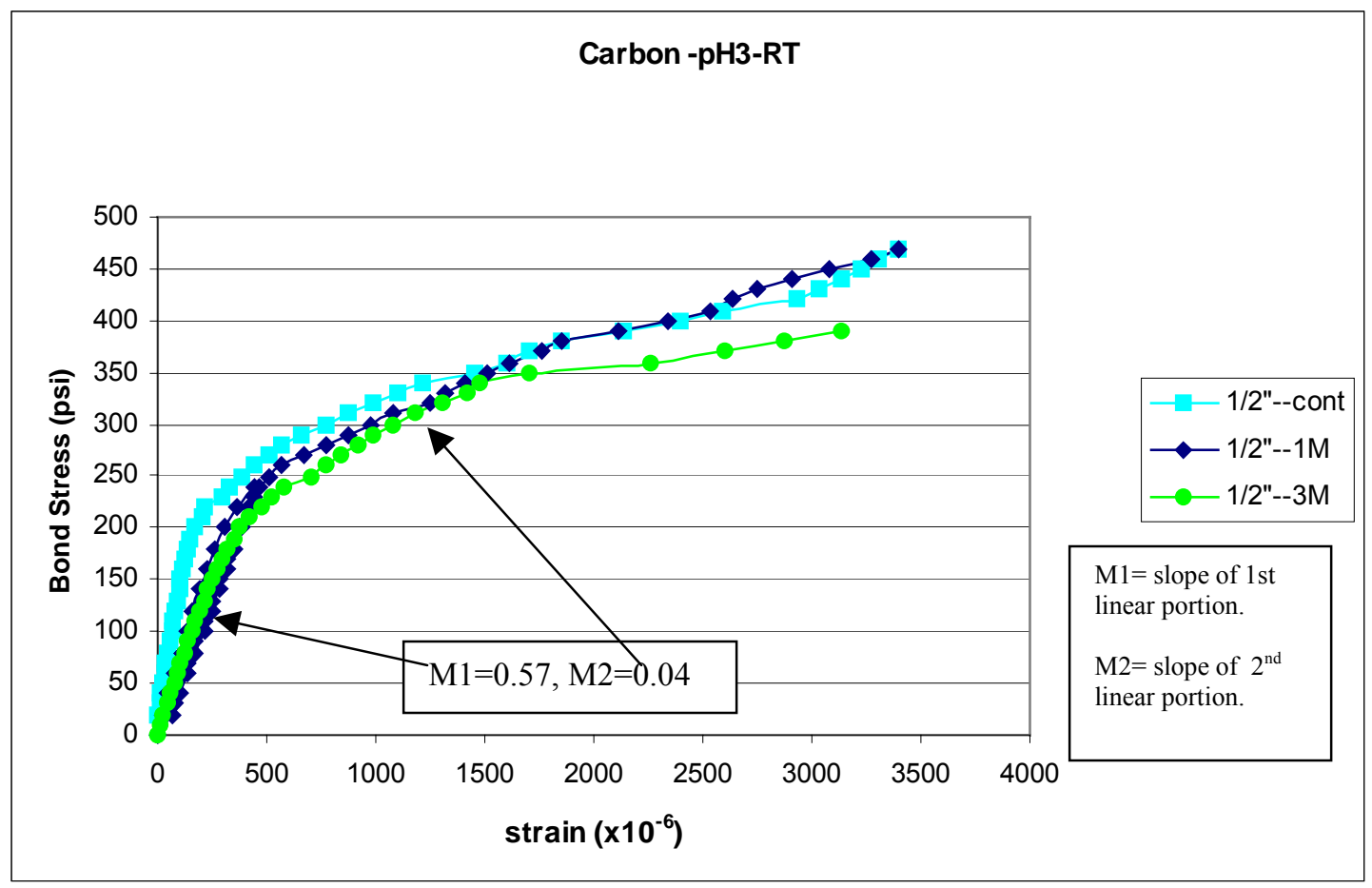

Figure 5.22 Strain Distribution for CFRP/pH3/RT

Figure 5.22 continues to show similar strain distribution between the aged and unaged specimens. The change in slope occurs around 200 psi stress and 400 microstrains. When comparing Figures 5.22 and 5.9 (same conditioning but different material), the bond behavior remains unchanged. However, the strains at the change in slope in the glass specimens are slightly higher than the strains at bifurcation for carbon at the same stress levels. This is because carbon has a higher stiffness than glass. Therefore it is thought that the initial slope can be related to a combination of the material plus the concrete strength. 


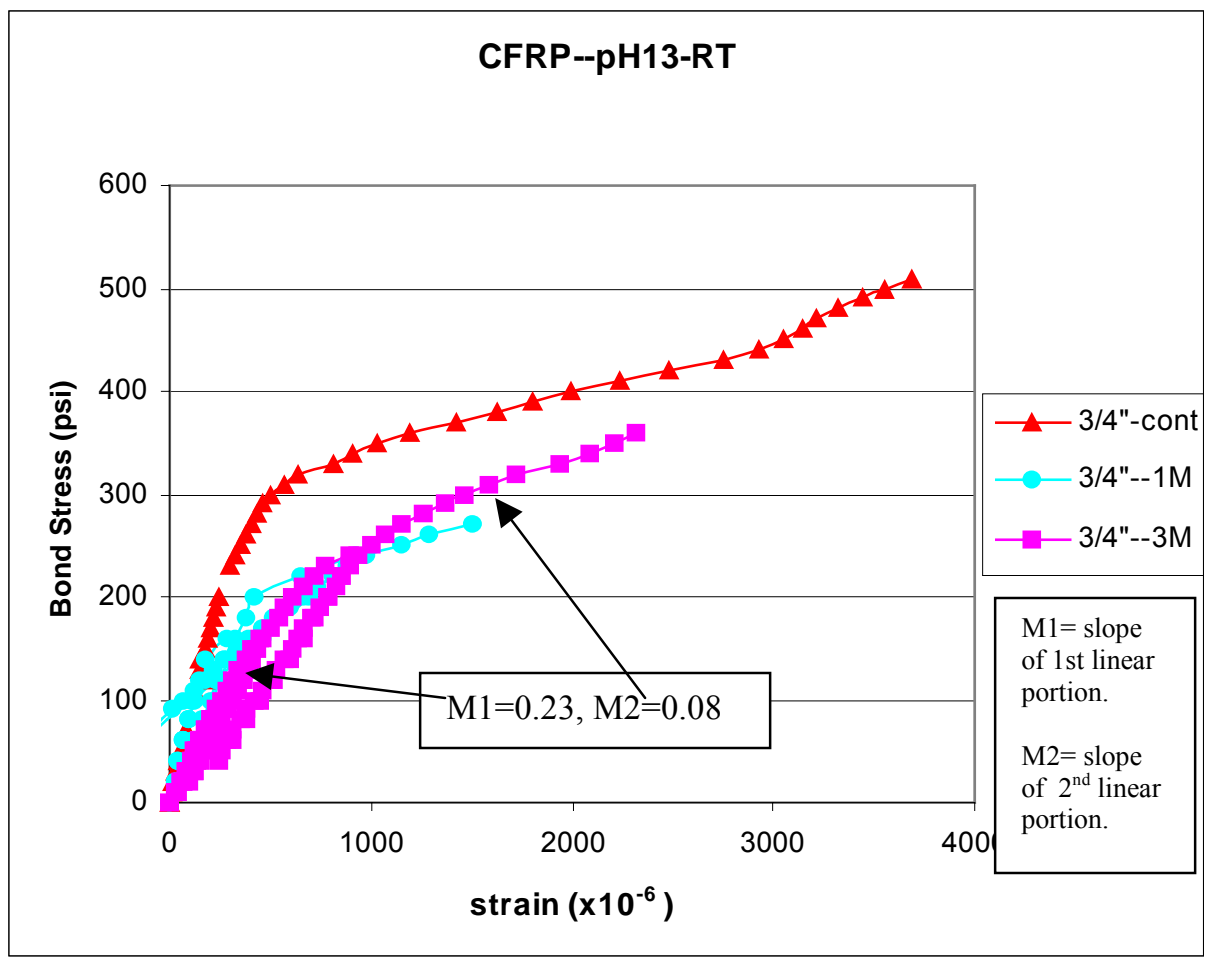

Figure 5.23 Strain Distribution for CFRP/pH 13/Room Temperature

The specimens shown in Figure 5.23 were aged at room temperature. Overall, the specimens showed similar distribution trends. When the aged specimens were tested it was decided to load them to certain stress levels and then drop back to zero to see if the strains would be recovered (same procedure used when testing specimens of Figure 5.15). This procedure demonstrates a loss in slope as the specimen is loaded to higher stress levels. This can be related to permanent set in strain beyond a certain threshold level, approximately 170-200 psi. 
Table 5.11 CFRP/pH 13/RT Stress and Strain Ratios

\begin{tabular}{|l|c|c|c|c|c|c|}
\hline $\begin{array}{l}\text { CFRP-pH 13 } \\
\text { Room Temp. }\end{array}$ & $\begin{array}{c}\text { A: Stress at } \\
\text { Bifurcation (psi) }\end{array}$ & $\begin{array}{c}\text { B: Stress at } \\
\text { Failure (psi) }\end{array}$ & $\begin{array}{c}\text { A / B } \\
\text { C: Strain at } \\
\text { Bifurcation }(\mu \varepsilon)\end{array}$ & $\begin{array}{c}\text { D: Strain at } \\
\text { Failure }(\mu \varepsilon)\end{array}$ & \begin{tabular}{c} 
C / D \\
\hline 1 month
\end{tabular} \\
\hline 3 months & 200 & 280 & .71 & 400 & 1503 & 0.27 \\
\hline
\end{tabular}

Table 5.12 CFRP/pH 3/RT Stress and Strain Ratios

\begin{tabular}{|l|c|c|c|c|c|c|}
\hline $\begin{array}{l}\text { CFRP-pH 3 } \\
\text { Room Temp. }\end{array}$ & $\begin{array}{c}\text { A: Stress at } \\
\text { Bifurcation (psi) }\end{array}$ & $\begin{array}{c}\text { B: Stress at } \\
\text { Failure (psi) }\end{array}$ & $\begin{array}{c}\text { A / B } \\
\text { C: Strain at } \\
\text { Bifurcation }(\mu \varepsilon)\end{array}$ & $\begin{array}{c}\text { D: Strain at } \\
\text { Failure }(\mu \varepsilon)\end{array}$ & C / D \\
\hline 1 month & 200 & 470 & .43 & 312 & 3400 & 0.09 \\
\hline 3 months & 200 & 390 & .51 & 392 & 3150 & 0.12 \\
\hline
\end{tabular}

Tables 5.11 and 5.12 give the ratios of the stress and strain at the points of bifurcation to the values at failure. Here again, these values demonstrate that the bifurcation takes place at approximately $50 \%$ of ultimate load. 


\subsubsection{Acid and Alkaline—Freeze-thaw}

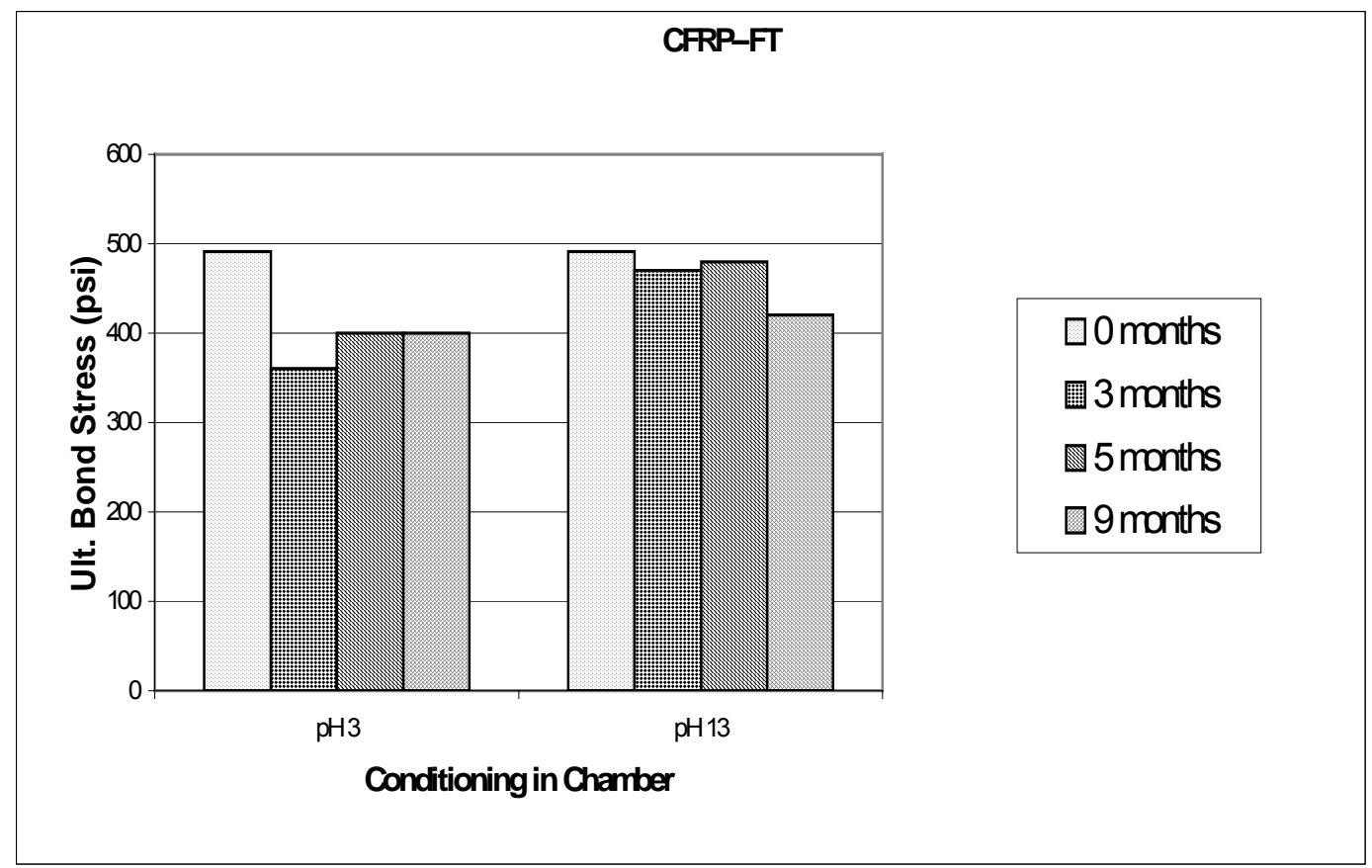

Figure 5.24 Effects of pH on Bond Strength Between CFRP and Concrete

Figure 5.24 shows the results for carbon specimens soaking in solutions at freezethaw conditions. Note that the five and nine month specimens did not undergo a sustained load while conditioning. The results for acid are showing an initial decrease in bond strength followed by an increase. The acid also appears to have a greater effect on the bond strength than alkaline solution. The acid soaked specimens are showing an average decrease in strength of $15 \%$ when compared to the alkaline soaked ones. When compared to results at room temperature (Figure 5.21), the acid soaked specimens are showing a lower bond strength than those conditioned in the chamber (freeze-thaw). 


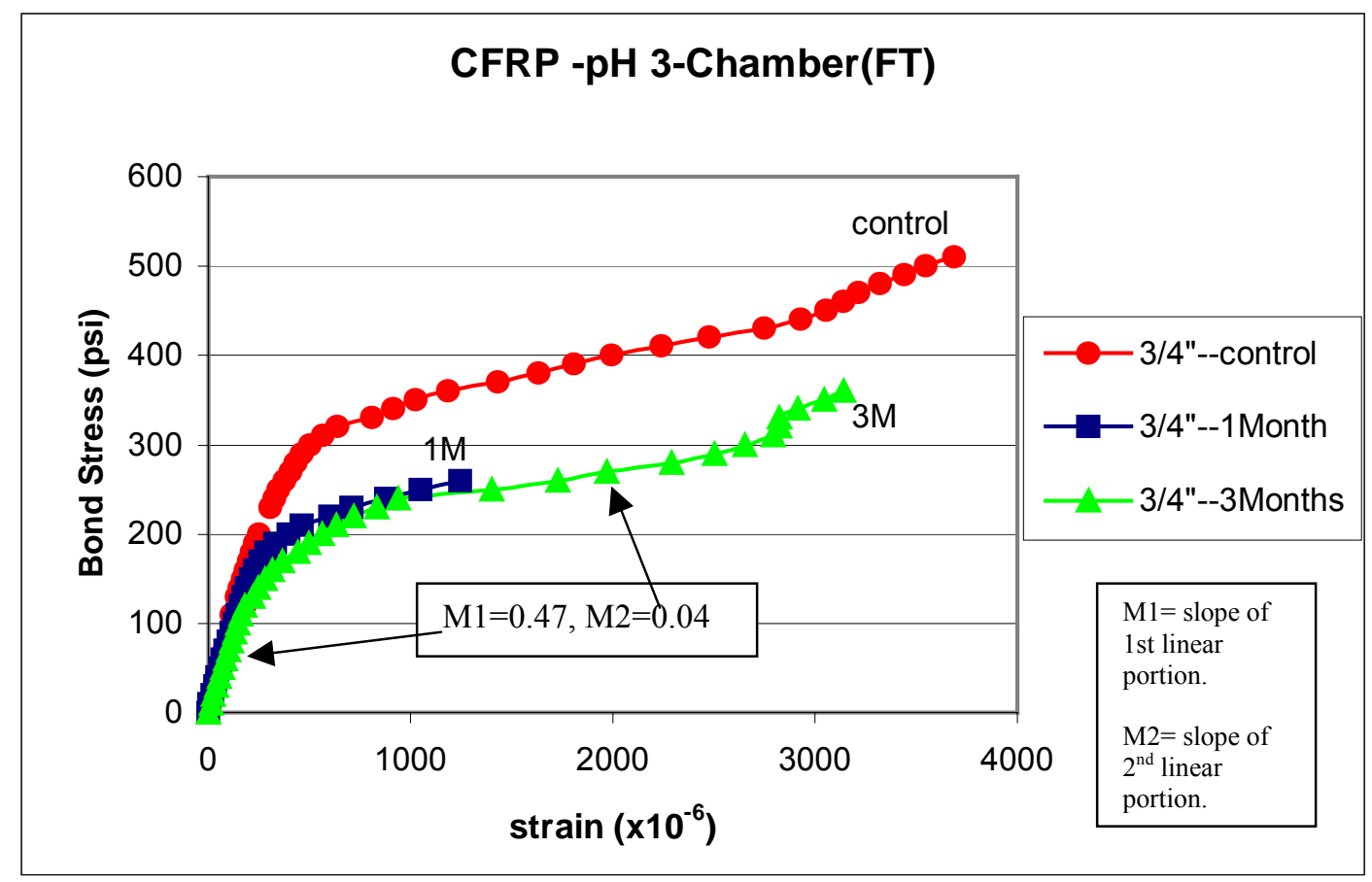

Figure 5.25 Strain Distribution for CFRP/pH3/chamber

In Figure 5.25 the slope of the linear portion of the lines can be related to the CFRP strips, the resin and the concrete strength. However, after the bifurcation point, the slope is thought to be directly related to the resin stiffness only. The aging does not seem to have an effect on the strain distribution. However, the slopes for the aged specimens change at a lower stress level than that of the unaged specimens. This can be due to moisture causing the resin to plasticize. 


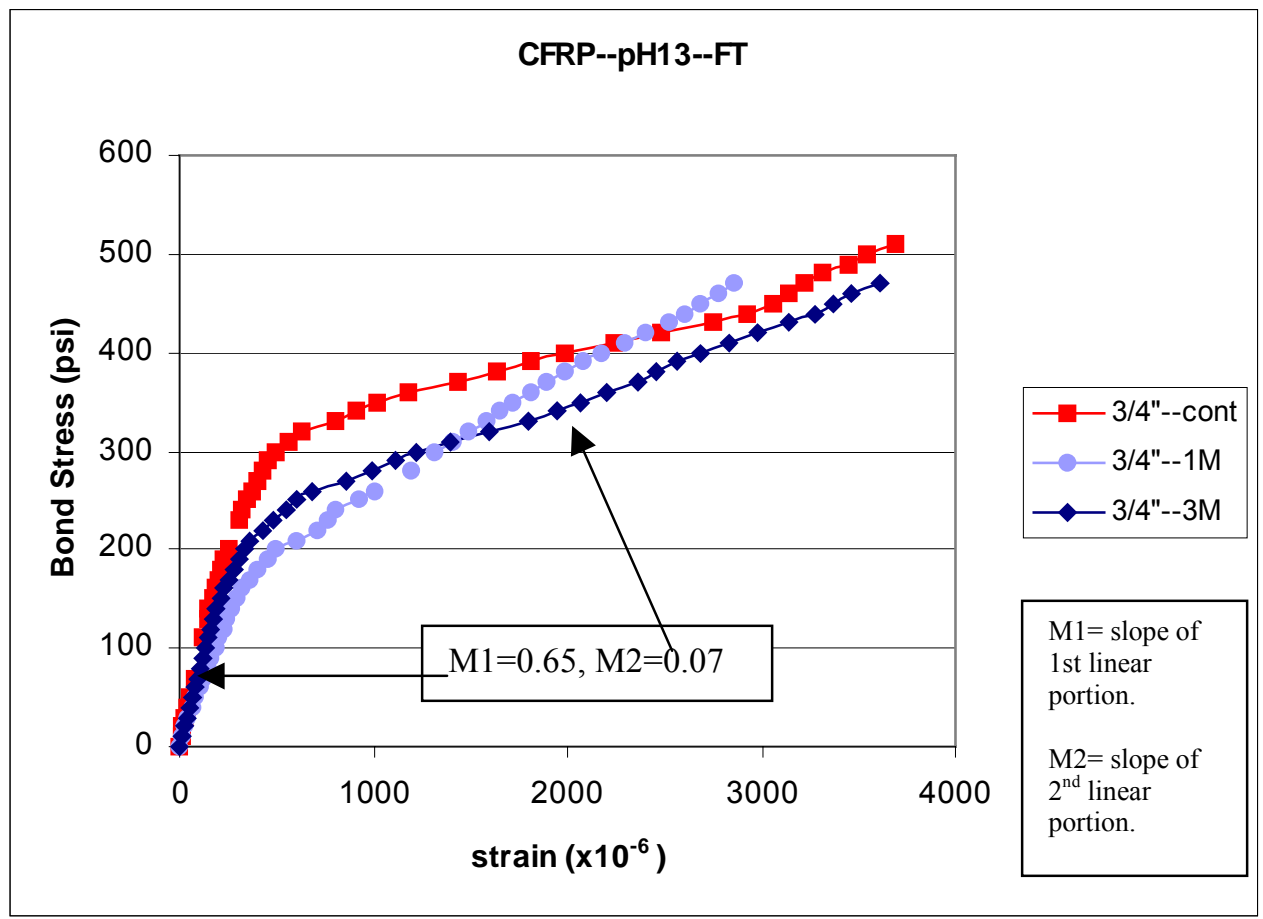

Figure 5.26 Strain Distribution for CFRP/pH 13/FT

In figure 5.26 the strain distribution for the aged specimens appears to be unchanged. The increase in strain at a lower bond stress is again due to the amount of moisture in the conditioned specimens, causing the adhesive to plasticize sooner than in the control specimen. 


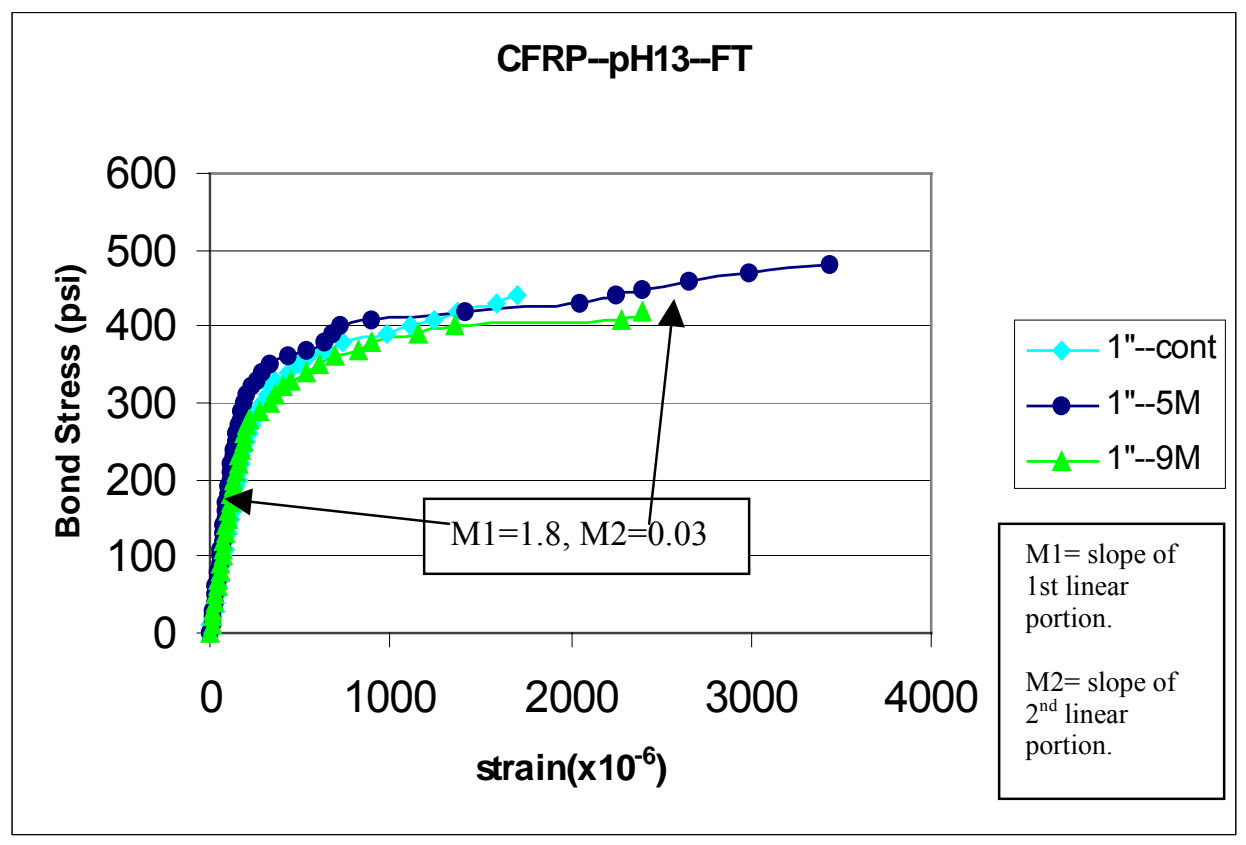

Figure 5.27 Strain Distribution for CFRP/pH 13/FT

Figure 5.27 depicts the results of the five and nine month aged specimens soaked in an alkaline solution and placed in the chamber. These specimens were aged without any sustained load and appear to be unharmed by the conditioning scheme. 
Table 5.13 CFRP/pH 13/FT Stress and Strain Ratios

\begin{tabular}{|l|c|c|c|c|c|c|}
\hline CFRP-pH 13 & $\begin{array}{c}\text { A: Stress at } \\
\text { Freeze-thaw }\end{array}$ & $\begin{array}{c}\text { B: Stress at } \\
\text { Bifurcation (psi) }\end{array}$ & Failure (psi) & A B & $\begin{array}{c}\text { C: Strain at } \\
\text { Bifurcation }(\mu \varepsilon)\end{array}$ & $\begin{array}{c}\text { D: Strain at } \\
\text { Failure }(\mu \varepsilon)\end{array}$ \\
\hline 1 month & 165 & 470 & .35 & 300 & 2856 & 0.11 \\
\hline 3 months & 200 & 470 & .43 & 300 & 3605 & 0.08 \\
\hline
\end{tabular}

Table 5.14 CFRP/pH 13/FT Stress and Strain Ratios

\begin{tabular}{|l|c|c|c|c|c|c|}
\hline CFRP-pH 13 & $\begin{array}{c}\text { A: Stress at } \\
\text { Freeze-thaw }\end{array}$ & $\begin{array}{c}\text { B: Stress at } \\
\text { Bifurcation (psi) }\end{array}$ & Failure (psi) & C: Strain at & D: Strain at & C / D \\
& 300 & 480 & .63 & 220 & 3400 & 0.06 \\
\hline 5 month & 280 & 420 & .67 & 250 & 2313 & 0.11 \\
\hline 9 months & & & & & & \\
\end{tabular}

Table 5.15 CFRP/pH 3/FT Stress and Strain Ratios

\begin{tabular}{|l|c|c|c|c|c|c|}
\hline CFRP-pH 3 & $\begin{array}{c}\text { A: Stress at } \\
\text { Freeze-thaw }\end{array}$ & $\begin{array}{c}\text { B: Stress at } \\
\text { Bifurcation (psi) }\end{array}$ & Failure (psi) & C: B & $\begin{array}{c}\text { D: Strain at } \\
\text { Bifurcation }(\mu \varepsilon)\end{array}$ & \begin{tabular}{c} 
Failure $(\mu \varepsilon)$ \\
\hline 1 month
\end{tabular} \\
\hline 3 months & 170 & 260 & .77 & 380 & 1200 & 0.32 \\
\hline
\end{tabular}

Tables 5.13-5.15 give the ratios of the stress and strain at the point of bifurcation to those at failure. Here again the average stress ratio is $55 \%$ and the strain is about $10 \%$. 


\subsubsection{Freeze-thaw (FT)}

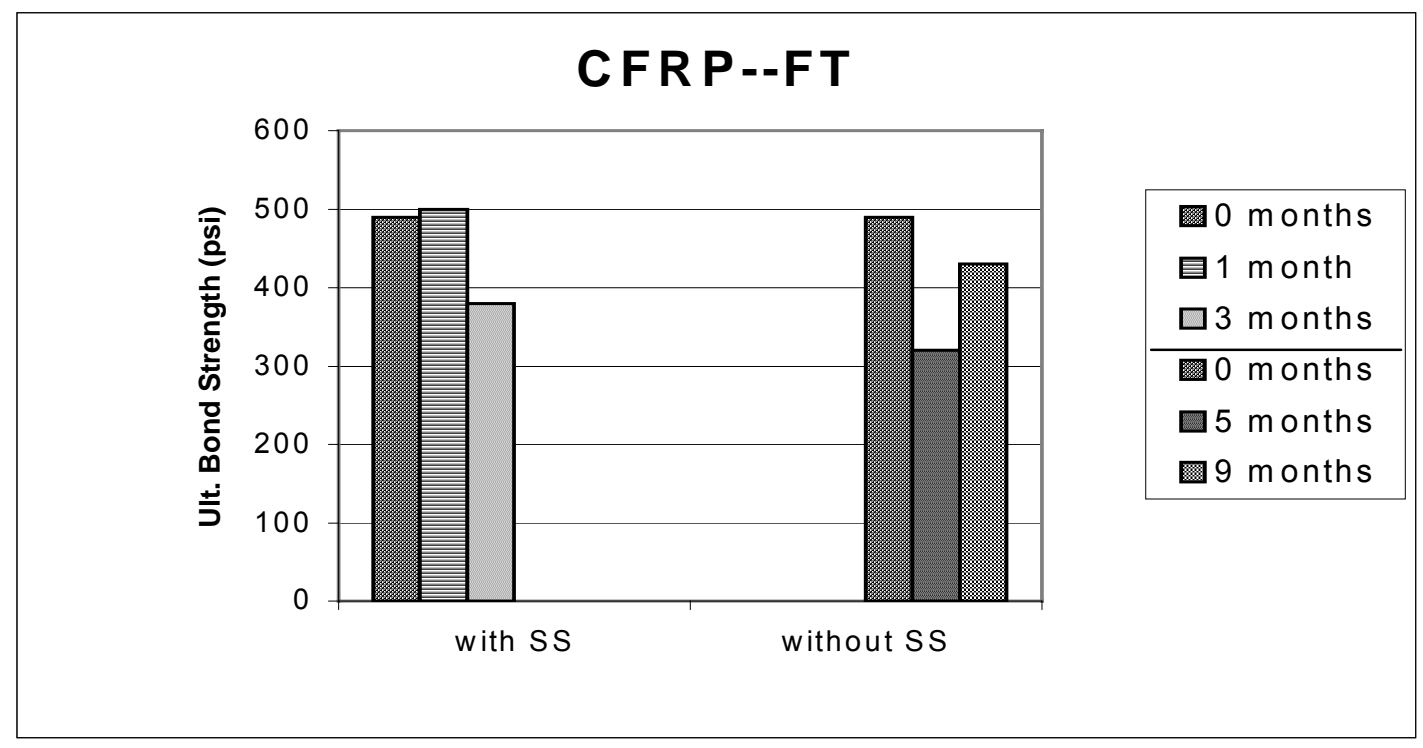

Figure 5.28 Effects of Aging on Bond Strength Between CFRP and Concrete

Figure 5.28 gives results for carbon specimens placed in the chamber with and without sustained stress. The sustained stress specimens show a continued decrease in strength with aging while the other specimens show a decrease followed by an increase. There are no significant changes in bond strength among sustained stress versus nonsustained stress specimens. 


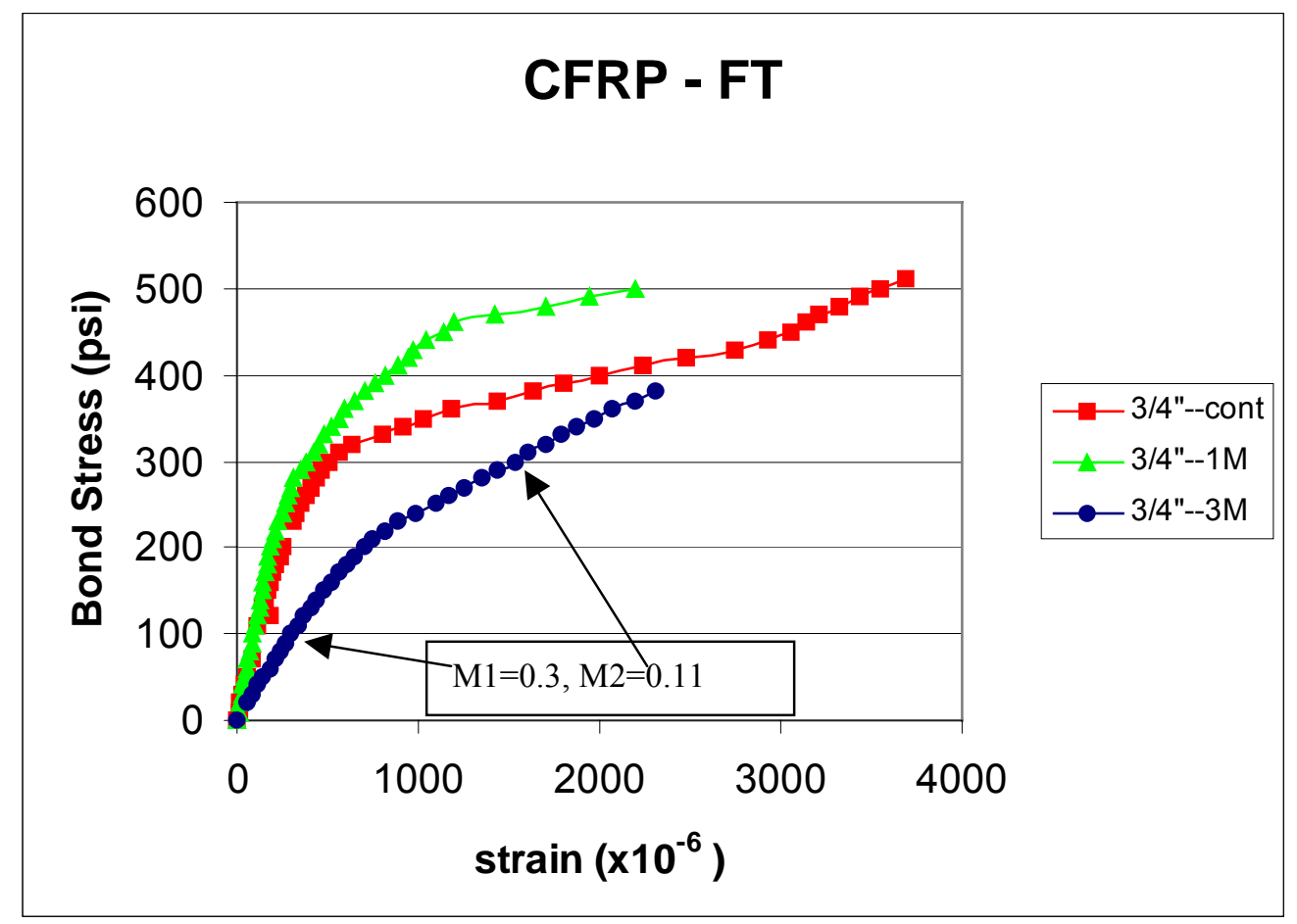

Figure 5.29 Strain Distribution for CFRP/FT

In figure 5.29 the specimen aged for three months under freeze-thaw conditions, is showing an increase in strain sooner than the others. This is because plasticization has caused the resin stiffness to decrease during aging due to temperature fluctuations and the sustained load or the resin may have begun to degrade while aging for a longer duration (3 months) under a sustained stress. 


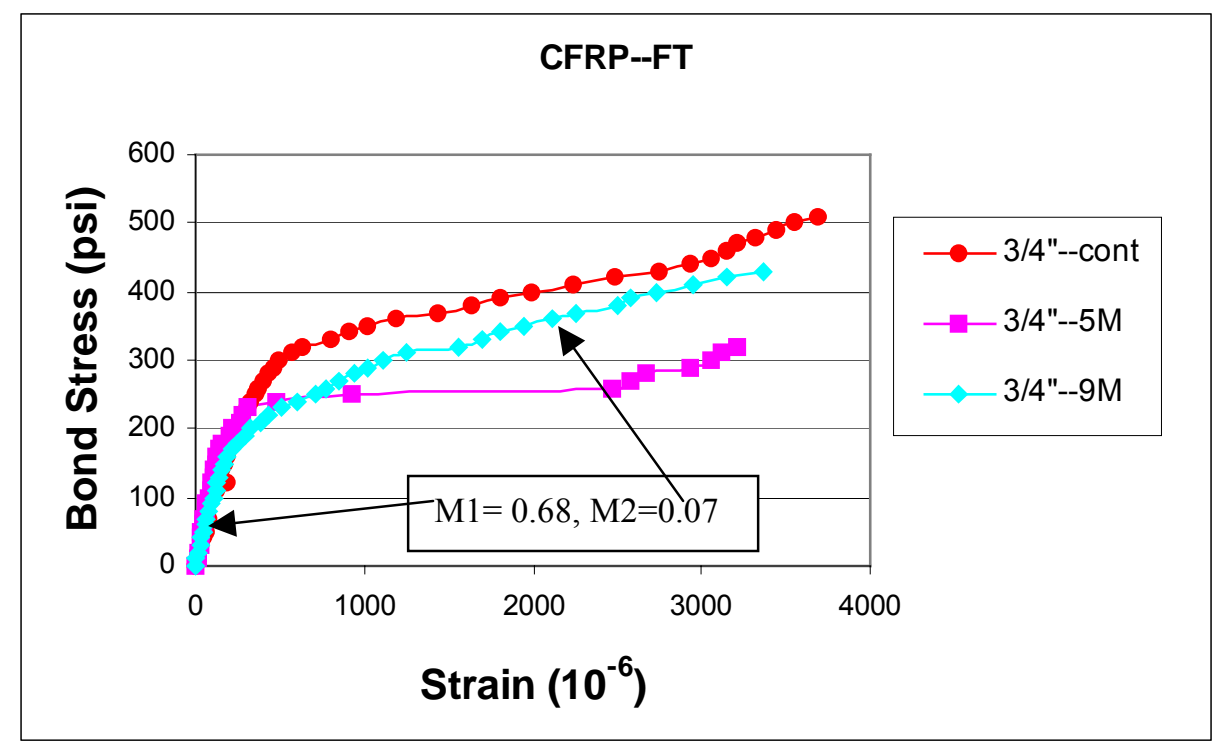

Figure 5.30 Strain Distribution for CFRP/FT

In figure 5.30 the strains are again showing similar distribution trends up to about 200 psi. If we look at the last few points on the specimen aged for 9 months, we can see that the lines of different aged specimens are running parallel to each other. This tells us that the strain increase with load is occurring at the same rate. But as we can see the aged curves fall below the control curve and therefore less load is required to reach the same levels of strain. This suggests that upon aging some degradation may be occurring in the fibers or the adhesive--most likely the adhesive.

In looking at figures 5.29-5.30, it can be decided that the freeze-thaw conditioning scheme as well as aging with or without a sustained stress are demonstrating no obvious adverse effects on the specimen behavior. The trend indicates that conditioning of specimens is only causing the adhesive to plasticize at a lower stress level than that in the unaged specimens. 
Table 5.16 CFRP/FT Stress and Strain Ratios

\begin{tabular}{|l|c|c|c|c|c|c|}
\hline CFRP-- & $\begin{array}{c}\text { A: Stress at } \\
\text { Freeze-thaw }\end{array}$ & $\begin{array}{c}\text { B: Stress at } \\
\text { Bifurcation (psi) }\end{array}$ & Failure (psi) & C: Strain at & D: Strain at & C / D \\
& 280 & 500 & .56 & 350 & 2190 & 0.16 \\
\hline 1 month & 200 & 390 & .51 & 700 & 2356 & 0.29 \\
\hline 3 months & & & & & & \\
\end{tabular}

Table 5.17 CFRP/FT Stress and Strain Ratios

\begin{tabular}{|l|c|c|c|c|c|c|}
\hline CFRP-- & $\begin{array}{c}\text { A: Stress at } \\
\text { Freeze-thaw }\end{array}$ & $\begin{array}{c}\text { B: Stress at } \\
\text { Bifurcation (psi) }\end{array}$ & Failure (psi) & C: Strain at & $\begin{array}{c}\text { D: Strain at } \\
\text { Bifurcation }(\mu \varepsilon)\end{array}$ & Failure $(\mu \varepsilon)$ \\
\hline 5 month & 170 & 320 & .53 & 200 & 3212 & 0.06 \\
\hline 9 months & 170 & 430 & .40 & 250 & 3362 & 0.07 \\
\hline
\end{tabular}

Tables 5.16 and 5.17 give the ratios of the stress and strain at the point of bifurcation to those at failure. Here again the average stress ratio is around $50 \%$ and the strain is about $15 \%$. 


\subsubsection{Water Conditioning}

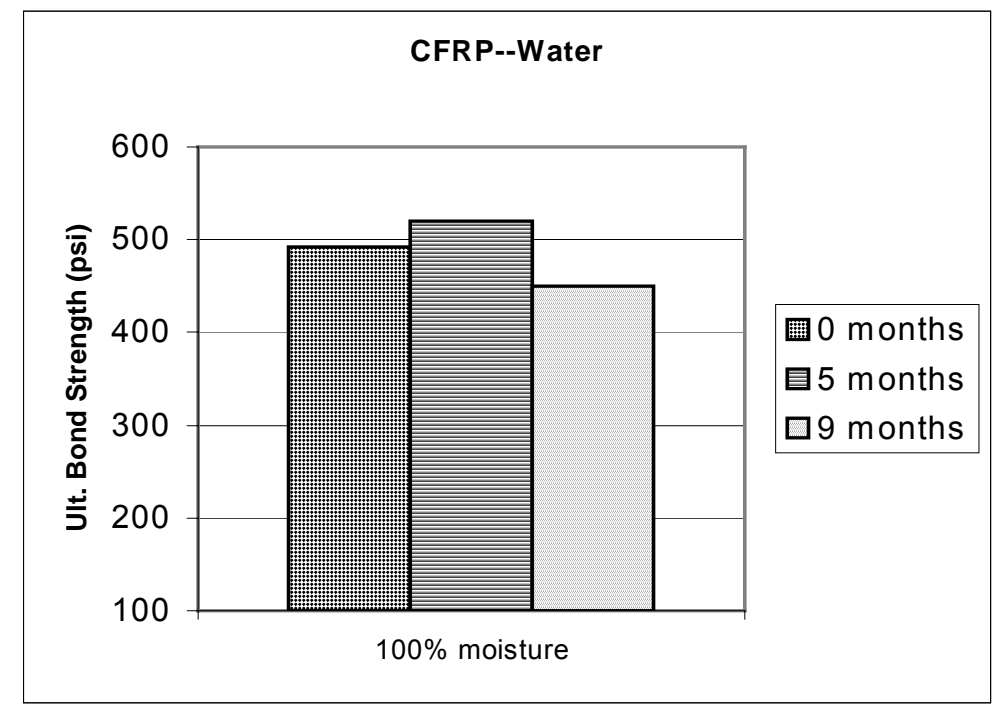

\subsection{Effects of Moisture on Bond Strength between CFRP and Concrete}

Results in Figure 5.31 show a slight increase in strength followed by a $12 \%$ decrease. The decrease can be attributed to moisture causing the resin to lose stiffness. Overall, the moisture effects are minimal and minimal (within $10 \%$ of original value) degradation is thought to be occurring. Exposure to water was done only for 5 and 9 month durations because it was felt that the specimens were getting enough exposure to moisture from other aging conditions, i.e. the $\mathrm{pH} 3$ and $\mathrm{pH} 13$ solutions. 
The following graph (Figure 5.32) is the stress vs. strain distribution for carbon specimens placed in water at room temperature for five and nine months. The strain readings were taken $3 / 4$ " back from the beginning of the bond area.

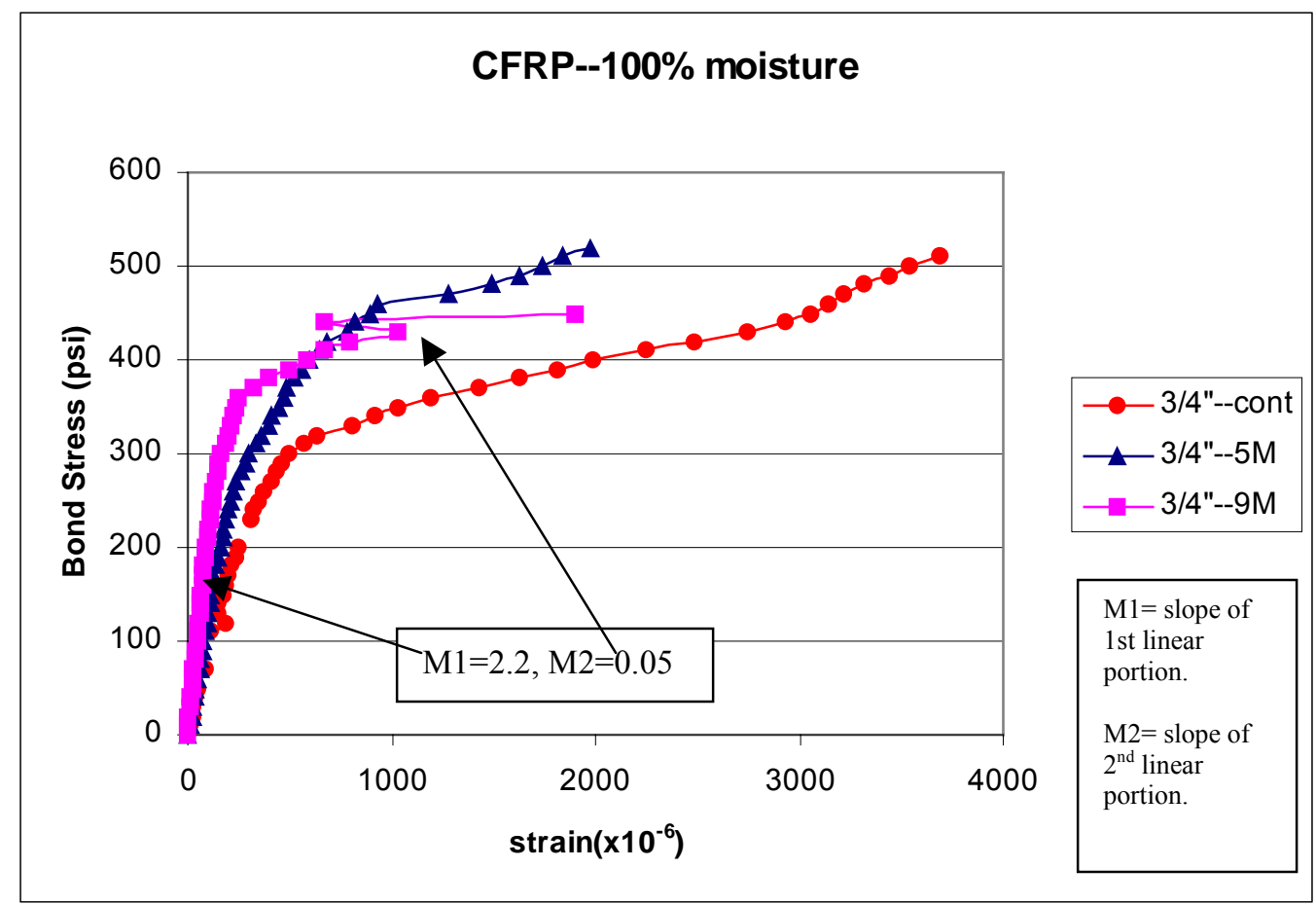

Figure 5.32 Strain Distribution of CFRP soaked in water

Figure 5.32 seems to be revealing that the strain distribution again is not altered upon aging. It can also be seen that the strain level at failure in the aged specimens is about half of what it is for the control specimen. The slope of the linear portion of the lines can be correlated to stiffness of CFRP and concrete substrate. 


\section{Table 5.18 CFRP/Water Stress and Strain Ratios}

\begin{tabular}{|c|c|c|c|c|c|c|}
\hline $\begin{array}{l}\text { CFRP and } \\
\text { Water }\end{array}$ & $\begin{array}{c}\text { A: Stress at } \\
\text { Bifurcation (psi) }\end{array}$ & $\begin{array}{l}\text { B: Stress at } \\
\text { Failure (psi) }\end{array}$ & $A / B$ & $\begin{array}{c}\text { C: Strain at } \\
\text { Bifurcation }(\mu \varepsilon)\end{array}$ & $\begin{array}{l}\text { D: Strain at } \\
\text { Failure }(\mu \varepsilon)\end{array}$ & $C / D$ \\
\hline 5 month & 280 & 510 & .55 & 300 & 2000 & 0.15 \\
\hline 9 months & 300 & 450 & .67 & 200 & 1965 & 0.10 \\
\hline
\end{tabular}

Table 5.18 gives the ratios of the stress and strain at the point of bifurcation to the stress and strain at failure. The average stress ratio is approximately $60 \%$ and the strain is about $15 \%$.

With the exception of a couple of specimens, average ratios are all coming out to be about the same for all aging conditions. The stress and strain ratios are approximately $50 \%$ and $15 \%$ respectively. 


\subsubsection{Natural Aging}

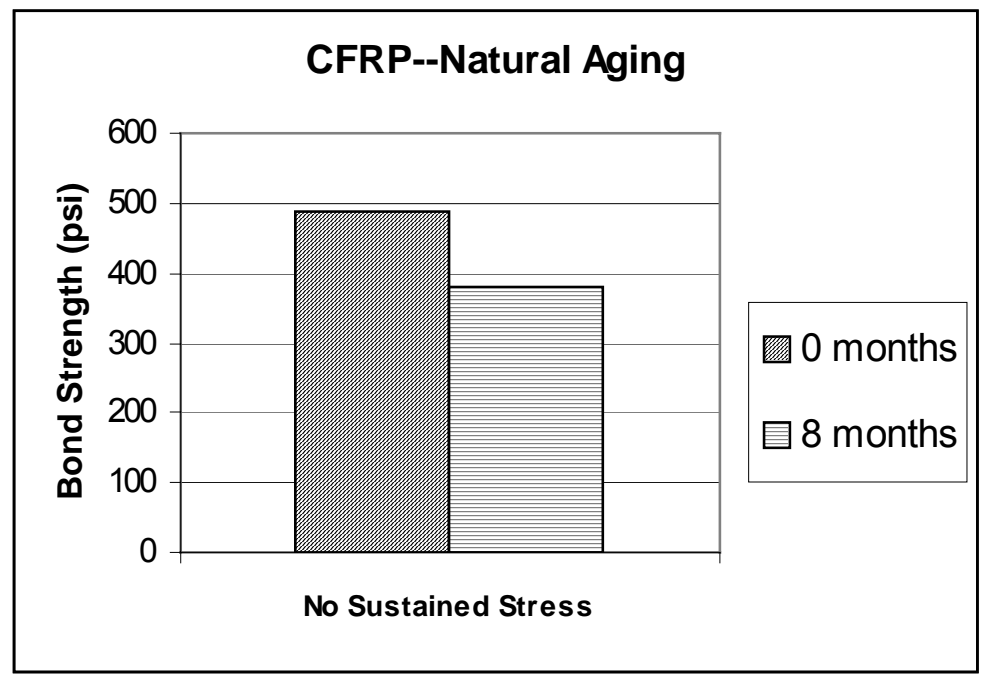

Figure 5.33 Bond Strength for CFRP/Natural Aging

Upon observing the strip at failure for the naturally aged specimens it was found that an inadequate amount of adhesive was applied when attaching the strips. The entire bond area was not coated thus resulting in a reduction of bond strength from $480 \mathrm{psi}$ to 380 psi. Such a reduction would not have occurred if a greater amount of adhesive would have been applied when attaching the strips. 


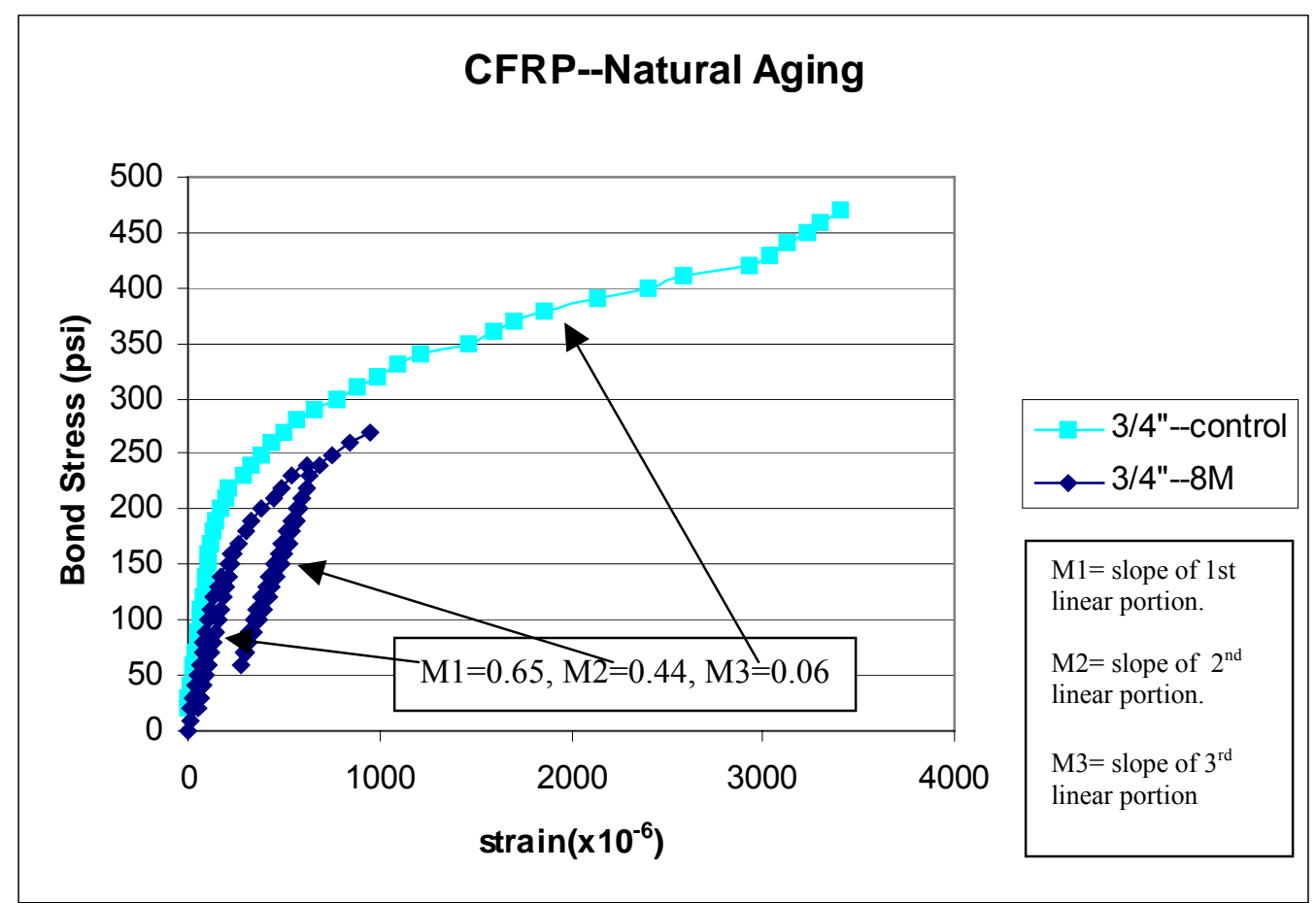

Figure 5.34 Strain Distribution for CFRP/Natural Aging

Figures 5.34 is the strain distribution for a naturally aged CFRP specimen. The premature failure was due to inadequate amounts of adhesive. Upon application of the strip an inadequate amount of pressure and adhesive were applied to the bond area. This suggests that an undetermined amount of pressure may need to be applied while curing of the adhesive is taking place. How to achieve this in the field is discussed in section 6.2.2.

Table 5.19 CFRP/Naturally Aged Stress and Strain Ratios

\begin{tabular}{|l|c|c|c|c|c|c|}
\hline CFRP-- & A: Stress at & B: Stress at & A / B & C: Strain at & D: Strain at \\
Natural & Bifurcation $(\mathrm{psi})$ & Failure $(\mathrm{psi})$ & & Bifurcation $(\mu \varepsilon)$ & Failure $(\mu \varepsilon)$ \\
Aging & & & & & & \\
\hline 8 months & 150 & 270 & .55 & 200 & 1000 & 0.20 \\
\hline
\end{tabular}

The ratios in table 5.19 are $55 \%$ and $20 \%$ for stress and strain respectively. 


\subsubsection{Beam Specimens}

Refer to section 4.6 and figure 4.14 for description of beam specimen.

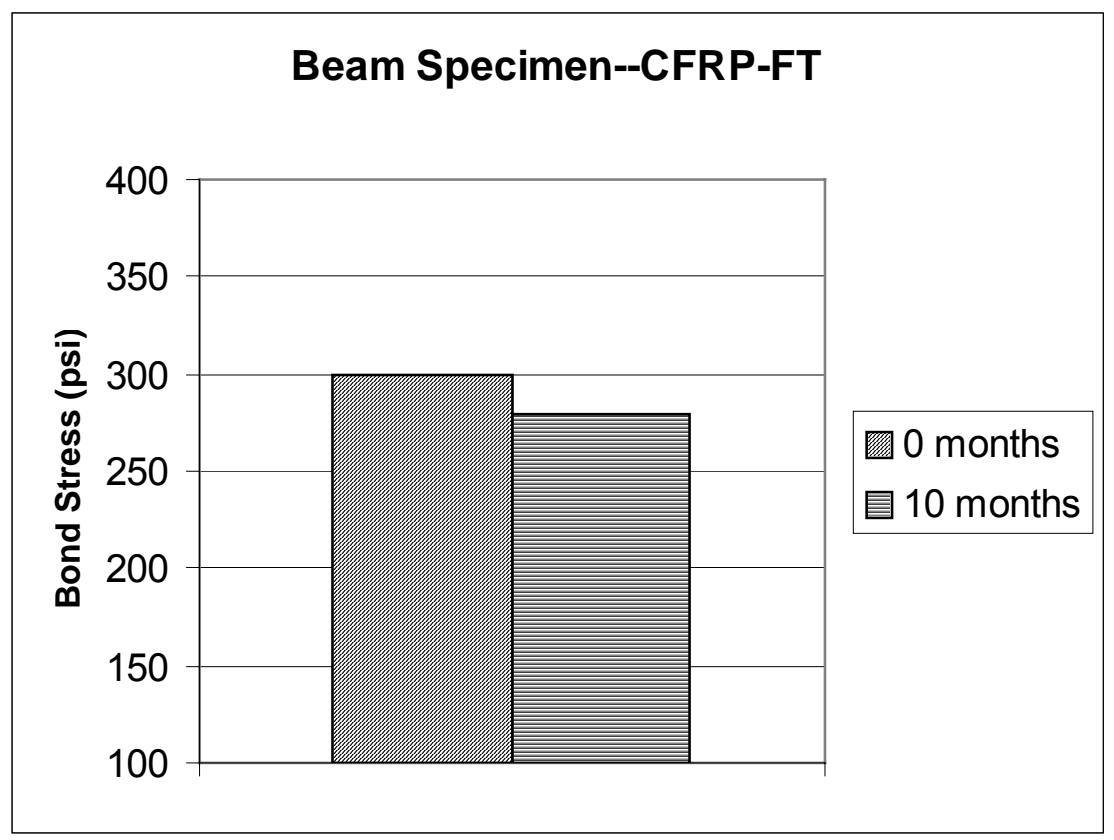

Figure 5.35 Bond Strength of Beam Specimen

Figure 5.35 shows bond stress versus number of months of aging for the beam specimens that were placed inside the environmental chamber for 10 months. The value of $280 \mathrm{psi}$ is the average of the ultimate bond strength of all the beam specimens aged under freeze-thaw conditions. Figure 5.35 shows a slight decrease in bond strength from 300 psi to 280 psi, i.e. $6-7 \%$ reduction under freeze-thaw conditions. It should be noted that this specimen sat in the Major Units Lab at room temperature for many months $(\approx 18$ months) before it was placed into the chamber. This may suggest that the specimen could have experienced small amounts of degradation while sitting at room temperature before being placed in the chamber. 


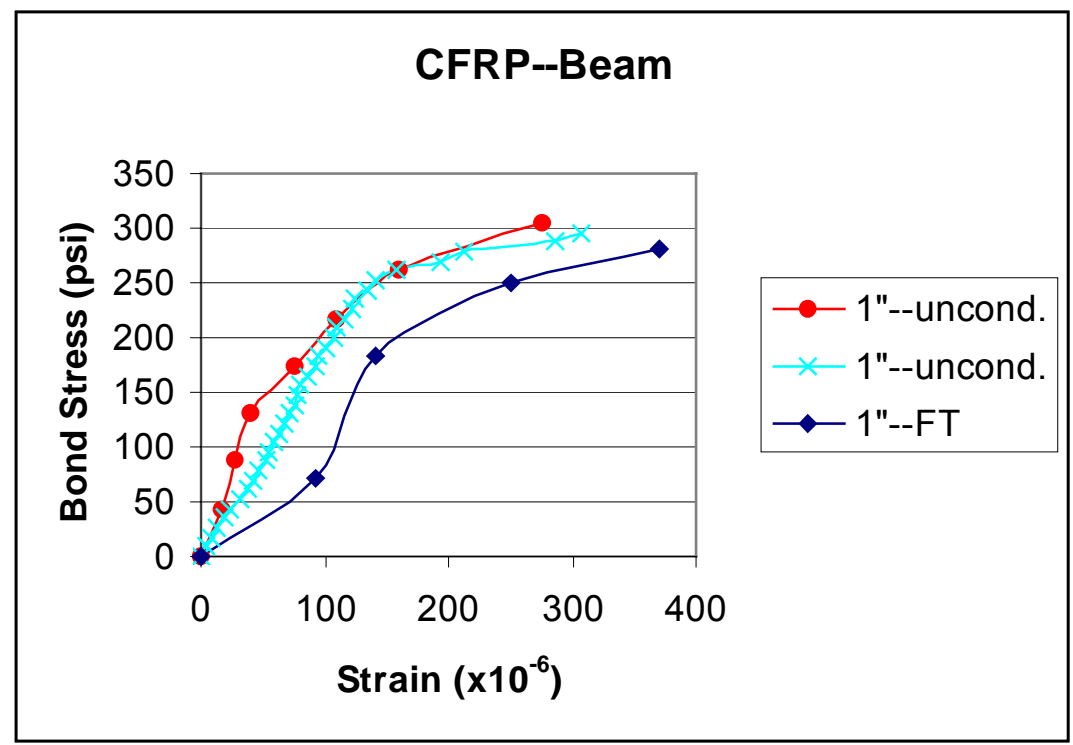

Figure 5.36 Strain Distribution of Beam Specimens

Figure 5.36 shows the results from the beam specimens that were tested. The two lines marked "uncond." are two of the specimens that were tested upon finding these leftover specimens in the Major Units Laboratory for over two years. The third line represents the results from placing a couple of specimens in the chamber. This was the only conditioning done to these specimens. The results of the unaged specimens show that the lines follow much the same pattern, with the aged line being shifted a little, which can be attributed to resin plasticization.

Table 5.20 Ratios for CFRP/Freeze-thaw Aged Beam Specimens

\begin{tabular}{|l|c|c|c|c|c|c|}
\hline CFRP-FT & $\begin{array}{c}\text { A: Stress at } \\
\text { Beam }\end{array}$ & $\begin{array}{c}\text { B: Stress at } \\
\text { Bifurcation (psi) }\end{array}$ & Failure (psi) & A / B & $\begin{array}{c}\text { C: Strain at } \\
\text { Bifurcation }(\mu \varepsilon)\end{array}$ & Failure $(\mu \varepsilon)$ \\
\hline 10 month & 170 & 270 & .63 & 140 & 370 & 0.38 \\
\hline
\end{tabular}

The ratios in table 5.20 are for the beam specimens. The ratios are a little higher when compared to previous results but this can be attributed to the low strength of the mortar. 


\subsection{Failure Modes}

Through the testing and evaluation of over 100 test specimens through this program, better understanding of the bond response of cube and beam specimens, failure modes have been noted. By looking at failure type, we may be able to determine the force transfer between FRP strips and concrete. We can also look at the adhesive bond and see how much, if any, of the failure is occurring within the adhesive. Theoretically, if degradation of the adhesive is occurring then we should see a failure along the bond line of the FRP strip and concrete. If the failure is occurring within the FRP material somewhere along the strip then we can conclude that the material is degrading upon aging. For the glass specimens soaked in alkaline solutions, this was the case. If a cohesive failure is occurring in concrete, then this tells us that the strain induced in concrete is too large for the material to withstand. Thus, a closer look at the strain to failure would give us a better idea of failure behavior and type. In order to use these strain readings for concrete, we must assume that the adhesive is fully transferring the stresses between the composite and concrete.

It was found in most every case, that a cohesive failure of concrete was occurring just beneath the bonded area [3]. In most cases only a thin layer of concrete remained attached to the strip after failure. Concrete located at the beginning of the bond (i.e. where the highest strain occurs) would break and remain adhered to the strip. This meant that a small amount of bending was occurring with initial compression. This suggests a compressive failure of concrete coupled with bending of concrete. This can be better explained in reference to the following diagram (Fig. 5.37a). As exemplified by the configuration shown, the load path eccentricity is not obvious, and there may be a 
tendency to assume that peel stresses are not present because, as a result of lateral symmetry, there is no overall bending. However, a little reflection brings to mind the fact that while the load in the symmetric lap joint flows axially through the central adherend prior to reaching the overlap region, there it splits in two directions, flowing laterally through the action of bond shear stresses to the two outer adherends. Thus eccentricity of the load path is present. As seen in Fig. 5.37b, the shear force $\left(\mathrm{F}_{\mathrm{SH}}\right)$ produces a component of the total moment about the neutral axis of the upper adherend equal to $\mathrm{F}_{\mathrm{SH}}{ }^{*} \mathrm{t} / 2$ where $\mathrm{t}$ is the thickness of the upper adherend. The peel stresses have to be present to react the moment produced by the offset of $\mathrm{F}_{\mathrm{SH}}$ about the neutral axis of the outer adherend [16].

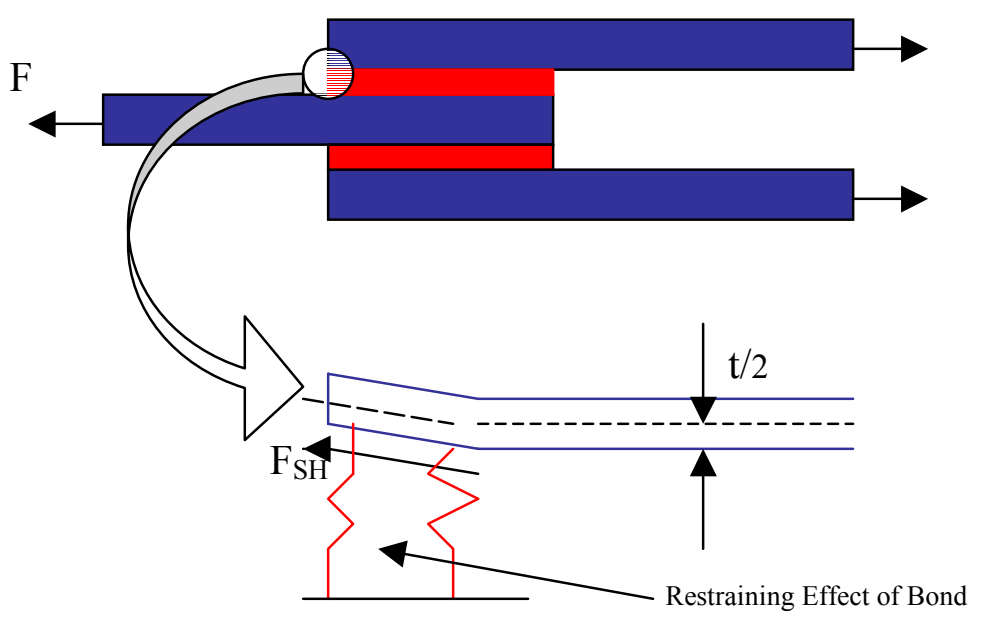

Figure 5.37 a

Figure $5.37 \mathrm{~b}$

The following pictures show how the failure looked. 


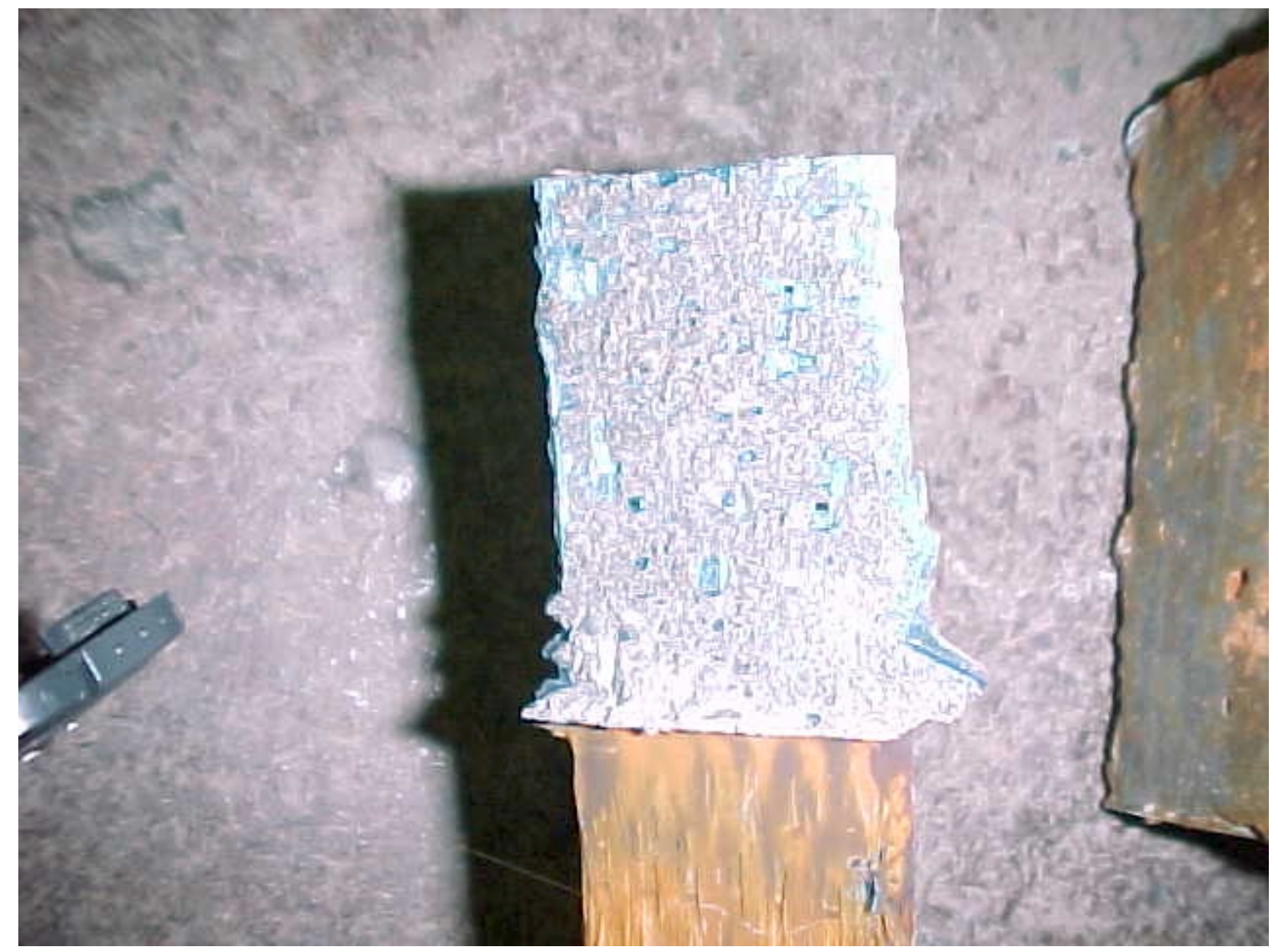

Figure 5.38 Thin Layer of Concrete Remaining on Strip

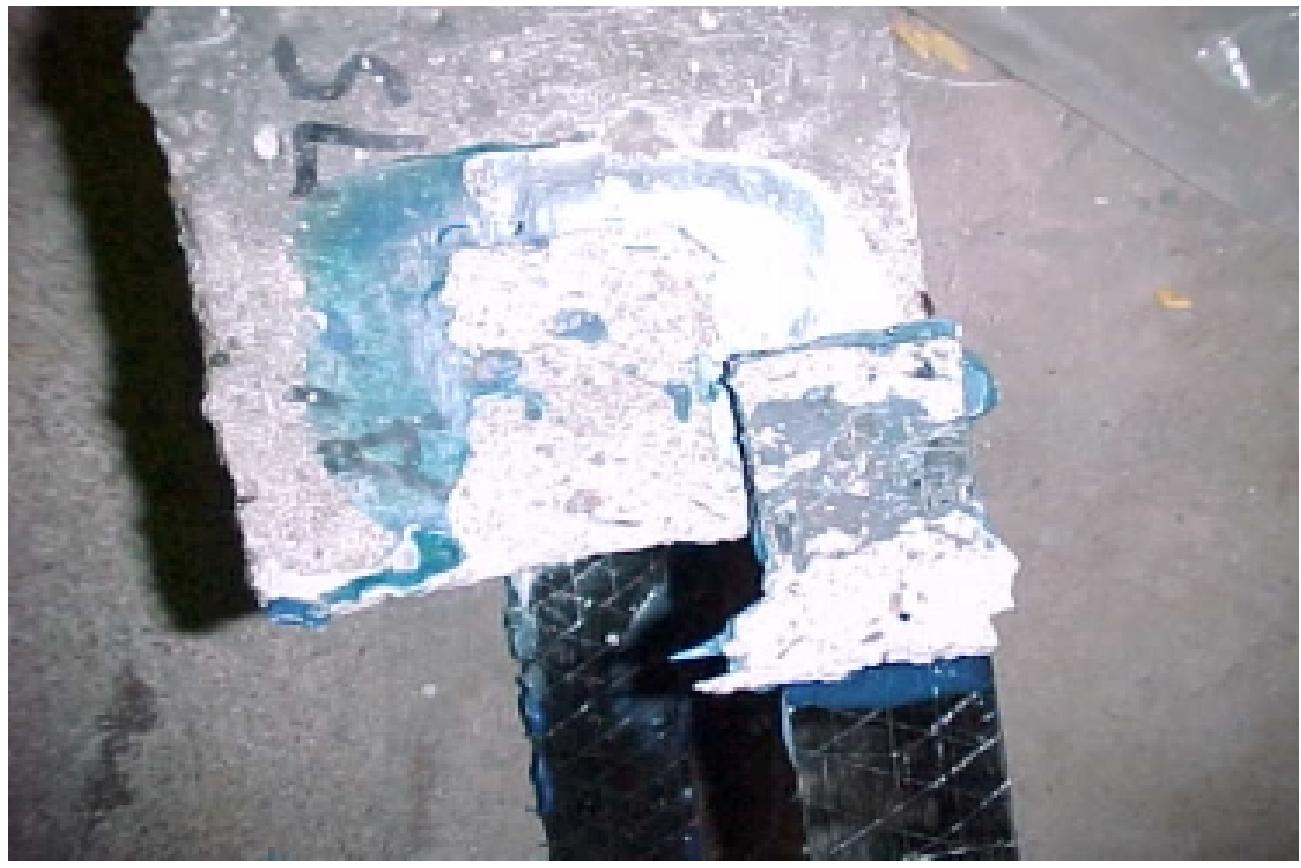

Figure 5.39 Concrete Remaining on Strip Where Strains are the Highest 
The following calculation was performed in order to determine the amount of concrete in compression just beneath the bond area.

Elastic Modulus ( $\mathrm{E}_{\text {comp }}$ ) of the composite was first calculated:

$\mathrm{E}_{\text {comp. }}=10 \times 10^{6}\left[\mathrm{t}_{\text {glass }+ \text { epoxy }} / \mathrm{t}_{\text {glass }}\right]=5 \times 10^{6}$, where $10 \times 10^{6} \mathrm{psi}$ is the $\mathrm{E}$ value for glass and $t$ represents the thickness of composite (glass + fiber) and the thickness of glass alone as measured in the lab.

This value $\left(5 \times 10^{6} \mathrm{psi}\right)$ multiplied by the strain value at the point of plasticization on the strain distribution graph, $300 \times 10^{-6} \mathrm{in} / \mathrm{in}$ ) yields a tensile stress of $1500 \mathrm{psi}$. Therefore in order to satisfy the following equation, $\sigma=$ Force $/$ Area, or $1500 \mathrm{psi}=450 \mathrm{lbs} /\left[\mathrm{t}_{\text {comp }}+\left(\mathrm{E}_{\text {concrete }} / \mathrm{E}_{\text {comp }}\right) \mathrm{t}_{\text {concrete }}\right] 1.25$ in. where area is the cross-sectional area of the composite plus a small layer of concrete and 1.25 inches is the width of the cross-sectional area, the thickness of concrete $\left(\mathrm{t}_{\text {concrete }}\right)$ in compression would have to be approximately $1 / 4$ " thick. Upon failure the average concrete thickness that remained adhered to the strip was measured to be precisely $1 / 4$ " thick. 


\subsection{Discussion of Methods Used which May Affect Results}

\subsubsection{Test Set-Up}

For this research the concrete cubes were poured prior to deciding on standardized test methods. Though many possibilities were examined, the chosen set up (see section 4.4) was used for two reasons. First, we were limited by the equipment that was available to us. Second, many specimens need testing and therefore, we needed a set up that would be both accurate and efficient. The Baldwin machine was our best candidate. Upon this decision, the grips were made. In order to fasten the grips onto the specimens easily, it would require about a 6" space between the cubes. Thus a large space between the two cubes has to be maintained during testing. However, a large space here meant that there would be a larger margin of error when trying to center the strips onto the cubes; but the trade off would be that localized bending would be minimized.

Overall the set-up was good and test results would have been even more consistent had the cubes been more uniform. This uniformity was very hard to achieve due to the small size of the test specimens. The form work that was made available to us was ideal for constructing beams but not small cubes. 


\subsubsection{FRP Application}

When applying the strip to the concrete, pressure was applied over the bond area during the setting of the resin. The amount of pressure varied as different size plates were used for applying the pressure. The amount of pressure did not seem to have any effect on how well the bond performed. Whether the amount was small or large did not affect the amount of load the bond could withstand. Thus, how to apply such a pressure in the field becomes a concern. The method used now involves applying the FRP by use of paint rollers to uniformly coat the epoxy. The epoxy is sticky enough to hold the FRP in place while curing is taking place. Although this method is fine, it seems as though if uniform pressure could be applied during curing, then the amount of air voids would be reduced and thus a stronger and more adequate bond would suffice. Finding a way to achieve this in the field is something that needs to be contemplated. One idea that may work well when applying FRP to columns is to apply some kind of air pressure around the column via air bags. After the FRP is applied using the rollers then the air bag would be pumped with air, thus applying a uniform pressure around the FRP. Though this idea may need more thought, suggestions for practical field applications have to be made and tested to see if the application of uniform pressure during curing is making a significant difference. 


\subsection{Overall Discussion of Results}

In studying the results for failure modes of each specimen, it was discovered that most every tested specimen demonstrated a cohesive concrete failure. This reveals that the failure is driven by the strain to failure in concrete. In addition, surface strength of concrete with higher cement paste content has a role to play in the failure strain level. Yet another factor that appears critical is the in-plane bond force at the crack surface inducing moments and stresses due to eccentric force leading to bending tension failure in concrete (Figure 5.38). In every case the failure strain is around 2800-4000 microstrains although a few aged specimens had a failure strain below this range. Therefore, the failure strain range suggests that aging may be affecting the concrete more so than the adhesive.

A similar trend was also found in every specimen with reference to strain bifurcation point, i.e., the location on the strain distribution graph (see Figures 5.25-5.26) where the line begins to change slope, i.e. M1 and M2. This bifurcation point is very revealing in that it was found in every case to occur at approximately $300-500$ microstrains. However, the stress at the bifurcation point differed by about $10 \%$ between the two materials with carbon being slightly greater than glass due to its higher stiffness. Therefore, from a stiffness view point, the fibers seem to be playing a role in determining the slope of the initial line (M1). Because the strain levels at bifurcation are about the same in all specimens, dramatic change in slope value from M1 to M2 suggests that something may be occurring within the adhesive or the concrete. The suggestion here is that concrete and the composite with adhesive elongate together up to a certain strain level $(300-500 \mu)$ upon which the primer-adhesive begins to yield and loses structural 
compositness between concrete substrate and the FRP. Beyond the point of bifurcation, the strain distribution line begins a second linear slope (M2). This slope is a function of either the adhesive or the fibers. Because no failure is observed in fibers, the slope of the second linear portion of the line (i.e. M2, slope after bifurcation point) is thought to be solely related to the adhesive properties. The distribution proceeds until the strain in concrete is large enough to cause concrete to locally fail in compression. This can be proven in the following calculation:

$$
\begin{aligned}
& E_{C F R P}=33 \mathrm{Msi}\left(23 \times 10^{4} \mathrm{~N} / \mathrm{mm}^{2}\right) \\
& E_{\text {concrete }}=3.5 \times 10^{6} \mathrm{psi}
\end{aligned}
$$

Assume that the fiber volume fraction of composite strip is $60 \%$ of the load and that of the resin is $40 \%$.

Therefore, $\mathrm{E}_{\mathrm{CFRP}}=33 \mathrm{Msi}(.6)=19.8 \mathrm{Msi}$

The modular ratio of $\mathrm{E}_{\mathrm{CFRP}}$ to $\mathrm{E}_{\text {concrete }}$ is $19.8 / 3.5=5.66$

Strain in concrete from modular ratio and composite strip strain $=$

$5.66 \times\left(425 \times 10^{-6}\right)=.0024 \mathrm{in} /$ in where $425 \times 10^{-6}$ is the average strain where non-linearity begins, i.e. the point of bifurcation, and the value of $.0024 \mathrm{in} / \mathrm{in}$ is the strain induced in concrete at that location. Therefore, as the strain continues to increase and goes beyond the bifurcation point, the calculated value of .0024 in/in approaches the ultimate strain of concrete, $.003 \mathrm{in} / \mathrm{in}$. Then failure occurs.

A similar explanation was cited from a study by Maeda et al[14]. He suggests that at initial loading, i.e.at low bond strain levels, the adhesive transfers $100 \%$ of the 
force and demonstrates $100 \%$ structural compositeness. As the bond stress level increases, the bonding material (adhesive) begins to yield at the tip of the bonded area, i.e. near the edge of the concrete cube. As induced bond force increases, the effective bond area is getting shifted further back along the bond line. The material is again stiff until this new area begins to yield and then the effective bond area shifts back again; thus a kind of a hinge mechanism to failure is noted. This continues until a certain strain level is reached to a value wherein concrete is led to fail, either in pure concrete compression at the surface or through compression coupled with bending near the concrete surface; thus resulting in a cohesive concrete failure just beneath the effective concrete bond area. This is also in agreement with a similar study from Tysl et al [20].

In looking at the rate of strain distribution for carbon, the values found in this study are compared to values found in the study by Maeda [14]. The strain distribution values for this study were $120-130 \mu$ strains $/ \mathrm{mm}$. Maeda's values were $110-115$ $\mu$ strains $/ \mathrm{mm}$. The $10 \%$ difference can be correlated to the concrete strength; $4 \mathrm{ksi}$ in this study and $6 \mathrm{ksi}$ for Maeda. It is thought that the stronger the concrete, the stiffer it is and stiffer concrete leads to lower strain inducement. The strain distribution rate for glass in this study was $140-150 \mu$ strains $/ \mathrm{mm}$. The $14 \%$ difference between the two materials is the result of carbon having greater stiffness than glass.

When studying slopes M1 and M2, where M1 is the slope of the strain distribution line prior to bifurcation and M2 is the slope of the line after bifurcation. For glass the average value for M1 was $0.663 \times 10^{6} \mathrm{psi}$. For carbon the average value of M1 was 0.816 x $10^{6}$ psi. The $19 \%$ difference can be explained by carbon having greater stiffness than glass. When comparing M2, glass had an average value of $0.055 \times 10^{6} \mathrm{psi}$ as did carbon. 
This confirms that the initial slope of the stress-strain line, M1, is controlled by the fiber type, while M2 is independent of the fibers, however, directly related to the adhesive. Surprisingly, the shear stiffness value for epoxy adhesive given in the Euro-Code [17] is exactly the average value that we found for M2, $.055 \times 10^{6} \mathrm{psi}$.

\subsection{Correlation of Natural to Accelerated Aging}

Actual calibration of accelerated aging to natural weathering is not complete in this study. However, it is previously established that the reduction trends in parameters such as tensile and compressive strengths have a maximum value during initial periods of aging and the rate of reduction decreases with time and reaches an asymptotic value [13], [21]. The maximum reduction found of all parameters in this accelerated aging study was approximately $25 \%$. Therefore, the experimental info of this study coupled with the previous studies indicated that the $25 \%$ reduction is conservatively extrapolated to be about 30 years. 


\section{Chapter 6}

\section{Conclusions and Recommendations}

\subsection{General Remarks and Summary}

The conclusions in section 6.2 are based on the evaluation of mbrace epoxy adhesive system with glass and carbon fabrics bonded to concrete and subjected to accelerated aging methods. Results may vary with adhesives other than epoxies.

Identical trends in strain distribution and bond stress were noted in different test specimens and these trends coincided with those from other researchers. Overall, the results suggest that the adhesive is marginally affected by aging and in most situations unaffected by aging. The evaluation of test results led to the following conclusions.

\subsection{Conclusions}

- Average variations in bond strength of specimens (glass and carbon) under accelerated aging were found to be within $10 \%$ of unaged. Accelerated aging consisted of parameters such as $\mathrm{pH}$ change (3 to 13), temperature fluctuations (room temperature and freeze-thaw between $12^{\circ} \mathrm{F}-120^{\circ} \mathrm{F}$ ) and varied degree of sustained stress $(0 \%-20 \%)$.

$>$ Bond Strength variations of glass fabrics under natural weathering for 8 months were found to be less than $8 \%$ with a strength value of 380 psi. 
On average, bond strength reductions under accelerated aging were less than $10 \%$ even though small increase in strength was noted for samples conditioned for about 30 days. Specimens aged under freeze-thaw conditioning exhibited an additional 5 to $10 \%$ reduction in bond strength as compared to specimens aged under room temperature, which is attributed to temperature fluctuations slightly affecting the adhesive and pore water near the surface freezing and thawing leading to higher strength degradation.

- Bond strain distribution along the bond line was found to have a quadratic shape that was almost identical under different aging conditions.

- Bond stress-strain curves showed two distinct slope regions, M1 and M2. The first region ended between $40-60 \%$ of ultimate stress. Corresponding strain values where slope bifurcation was initiated, ranged from $300 \times 10^{-6}$ to $500 \times 10^{-6}$ in./in which depended on concrete ultimate stress and conditioning schemes. These strains were approximately $10 \%$ of the ultimate strain of concrete.

- Slope M1 for carbon fabric was $23 \%$ higher than that of glass fabric, whereas slope M2 was found to have similar slope in both carbon and glass fabric cases. This implies that the first region of the stress-strain curve, M1, is influenced by fiber type, whereas the second region, M2, is dominated by adhesive properties. Typical shear stiffness of epoxy adhesive is 55,000 psi [17], which is what the average slope of M2 was found to be. 
- In the bond stress vs. strain curves, slope of the initial line, M1, was found to be about 15 and 12 times higher than that of M2 respectively for carbon and glass fabric bonded to concrete.

- Sustained stress of $20 \%$ was found to have no noticeable effect on bond strength of both carbon and glass fabric with concrete. Bond Strength of specimens aged with $20 \%$ sustained stress was within $5 \%$ of those aged without sustained stress.

- The average bond strain at failure was found to be $.0035 \mathrm{in} / \mathrm{in}$ for both glass and carbon specimens and this was observed over a bond length of 2 inches. The failure mode was a cohesive failure in concrete just below the bonded area. A uniform layer of cement and aggregate particles from the surface of the bonded concrete area was found to be adhering to carbon and glass strips (see Figure 5.37). In most cases, chipping off of a small wedge of concrete, with an average dimension of $1 / 4$, was observed closest to the end (see Figure 5.38). It is concluded that an adhesive failure may have been observed if the aging were to be carried for a longer duration beyond 10 months.

- The strain distribution trend as well as the failure mode was similar in the beams (Figure 5.36) as for the cubes. However, the bond stress at failure was about $300 \mathrm{psi}$ in the beams and $400 \mathrm{psi}$ for the cubes. This is due to low strength mortar being used for the beams. 
- No confinement effects were investigated in this study. In reality, positive confinement effects may be noted through the proper use of transverse fabrics.

\subsection{Recommendations}

- Calibration of natural vs. accelerated aging of concrete beams with wraps is required.

- Initiation of failure and progression of failure needs to be understood

- Investigation of greater permeation of primers into concrete is suggested to improve the depth of concrete layer that is effective in resisting compressive forces near the concrete surface.

- $\quad$ Ease of application techniques of wraps needs to be developed

- Understanding of response of wrapped beams under combined bending, shear and torsion effects, i.e., how does a wrap perform under stress reversal, i.e., forces changing from tension to compression is needed.

- Investigation of ductility improvements of concrete as result of wrapping has to be researched more fully.

- Effect of confinement on bond strength using varying numbers of transverse wraps with different anchorage lengths has to be evaluated. 


\section{References}

1. Armstrong, K.B., "Effect of absorbed water in CFRP composites on adhesive bonding", Int. J. Adhesion and Adhesives, Vol. 16, No. 1, 1996, pg. 21-28.

2. Brosens, K., Van Gemert, D., "Anchoring Stresses Between Concrete and Carbon Fiber Reinforced Laminates", Proceedings of the Third International Symposium, Vol. 1, October, 1997. Belgium.

3. Green, M., and Bisby, L.A., "Effects of Freeze-thaw Action on the Bond of FRP Sheets to Concrete", Durability Of Fibre Reinforced Polymer (FRP) Composites For Construction. 1998. Queen's University, Kingston, Ontario Canada.

4. Horiguchi, T., Saeki, N., "Effect of Test Methods and Quality of Concrete on Bond Strength of CFRP Sheet", Proceedings of the Third International Symposium, Vol. 1, Oct., 1997.

5. Hota, V.S. GangaRao, Faza, S.S., and Vijay, P.V., "Behavior of Concrete Beams Wrapped with Carbon Tow Sheet", CFC report No. 95-196, April 95, submitted to Tonen Corporation, 1-1-1 Hitotsukashi, Chiyoda-ku, Tokyo, 100 Japan.

6. Javed, Sara., "Accelerated Aging in Concrete Beams Externally Bonded with Carbon Fiber Tow Sheet", CFC report No. 96-239, West Virginia University, 1996.

7. Kajorncheappunngam, Somjai, "The Effects of Environmental Aging on the Durability of Glass/Epoxy Composite", Dissertation submitted to the College of Engineering of West Virginia University.

8. Karbhari, V.M., and Xie, Ming., "Peel Test for Characterization of Polymer Composite/Concrete Interface", Journal of Composite Materials, Vol. 31, No. $18 / 1997$.

9. Karbhari, V.M., and M. Engineer, "Effect of Environmental Exposure on the External Strengthening of Concrete with Composites--Short Term Bond Durability", Journal of Reinforced Plastics and Composites, Vol. 15--December 1996.

10. Katsuki, T., Uomoto, T., Masuda, Y., and Maruyama, K., "Experimental Study on Deterioration of Adhesion Properties of Continuous Fiber Sheet", October/November ACI Proceedings, 1999.

11. Kinloch, A.J., “Adhesion and Adhesives", Science and Technology, Chapman and Hall, London. 1987. 
12. Kshirsagar, S., Lopez-Anido, R., and Gupta, R.K., "Accelerated Environmental Aging of FRP-Wrapped Concrete Columns", Department of Chemical Engineering, West Virginia University.

13. Litherland, K.L., Oakley, D.R. and Proctor, B.A. (1981), "The Use of Accelerated Ageing Procedures to Predict the Long Term Strength of GRC Composites," Cement and Concrete Research, Vol.11, pp.455-466.

14. Maeda, T., Asano, Y., Sato, Y., Ueda, T., Kakuta, Y., "A Study on Bond Mechanism of Carbon Fiber Sheet", Proceedings of the Third International Symposium, Vol. 1, Oct., 1997.

15. Muszynski, L.C. \& Sierakowski, R.L., "Durability of Carbon Fiber Reinforced Plastics (CFRP) as External Reinforcement of Concrete Beams", $50^{\text {th }}$ Annual Conference, Composite Institute The Society of Plastics Industry Inc. Jan. 30-Feb. $1,1995$.

16. Oplinger, D.W., Adhesive joints, Handbook of Composites 2nd Edition, Chapman and Hall, 1998, pp.610-666.

17. Structural Design of Polymer Composites-EUROCOMP Design Code and Handbook, Edited by John L. Clarke, 1996.

18. Thomson, T.A., Jr, "Flexural Strengthening of Concrete Beams using Externally Bonded Composite Fabrics: An Ultimate Strength and Durability Analysis", Thesis submitted to the Faculty of the University of Delaware in partial fulfillment of the requirements for the degree of Master of Civil Engineering, Fall 1994.

19. Tysl, S.R., Imbrogno, M., Miller, B.D., "Effect of Surface Delamination on the Freeze/Thaw Durability of CFRP-Reinforced Concrete Beams", Durability Of Fibre Reinforced Polymer (FRP) Composites For Construction. 1998.

20. Tysl, S.R., Imbrogno, M., Kovach, L., and Barbour, R., "Behavior of Concrete Beams Strengthened with Carbon Fiber Reinforced Polymers Subjected to Saturated, Cyclic Freeze-Thaw Conditioning", October/November ACI Proceedings, 1999.

21. Vijay, P.V., Aging Behavior of Concrete Beams Reinforced with GFRP bars, Ph.D. Dissertation submitted to WVU, May 1999.

22. http://WWW.mcelwee.net/html/properties of varıus material

Properties of Various Materials. 


\section{Appendix A}

Epoxy Properties and Specifications 
MBrace

System

$\underline{\text { Projects }}$

News

Evaluation

\& Design

Contractor

Network

$\underline{\text { Contact Us }}$

Home

\section{Carbon Fiber and E-Glass Reinforcement to Extend the Life of Concrete Structures}

Provided by Master Builders, Inc. and Structural Preservation Systems, Inc., the MBrace System is a comprehensive approach to bringing innovative strengthtening techniques to the construction industry. As a result of this strategic alliance between two industry leaders, MBrace is more than an economical, field-proven technology for supplemental structural reinforcing....it offers single source support from specification through completion.

\section{(if \\ Component Manufacturer \\ Founded in 1909, Master Builders, Inc. is a leading innovator in the development, manufacturing and marketing of construction chemicals. With the world's largest privat research and development center devoted exclusively to concrete, the company offers products that improve, protect and repair concrete. Master Builders, Inc. is the headquarters for the region of the Americas within MBT Holding, a worldwide network of construction chemical companies owned by SKW Trostberg AG. The companies operate as part of the SKW-MBT Construction Chemicals Group, the largest producer in the construction chemical marketplace.}

Master Builders Inc.

23700 Chagrin Boulevard

Cleveland, $\mathrm{OH} 44122$

\section{6-831-5500 or 800-MBT-9990}

Browse with

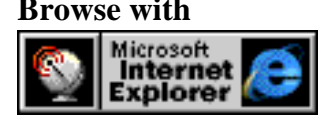

If you reached this page from a search engine and would like to visit our complete site, click here.

Revised: August 02, 1999

Copyright (C) 1999 Structural Preservation Systems, Inc. All rights reserved.
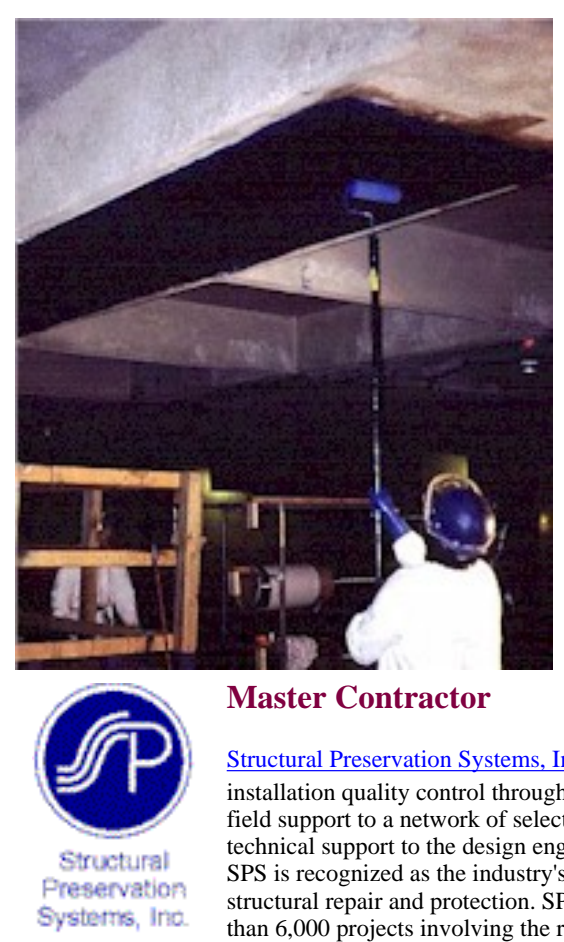

Master Contractor

Structural Preservation Systems, Inc. (SPS) provides installation quality control through contractor training and field support to a network of selected contractors and technical support to the design engineer. Founded in 1976, SPS is recognized as the industry's leading contractor in structural repair and protection. SPS has completed more than 6,000 projects involving the repair of concrete, masonry, timber, steel and soil. The company provides the experience and technical competence to solve a wide range of problems requiring surface repair, strengthening, stabilization, waterproofing and protection.

Structural Preservation Systems

7455-T New Ridge Road

Baltimore, MD 21076-3143

410-850-7000 or 800-899-1016 


\section{The MBrace System}

\section{$\underline{\text { Technology }}|\underline{\text { Components }}| \underline{\text { Features \& Benefits }}|\underline{\text { Applications }}| \underline{\text { Installation }}$}

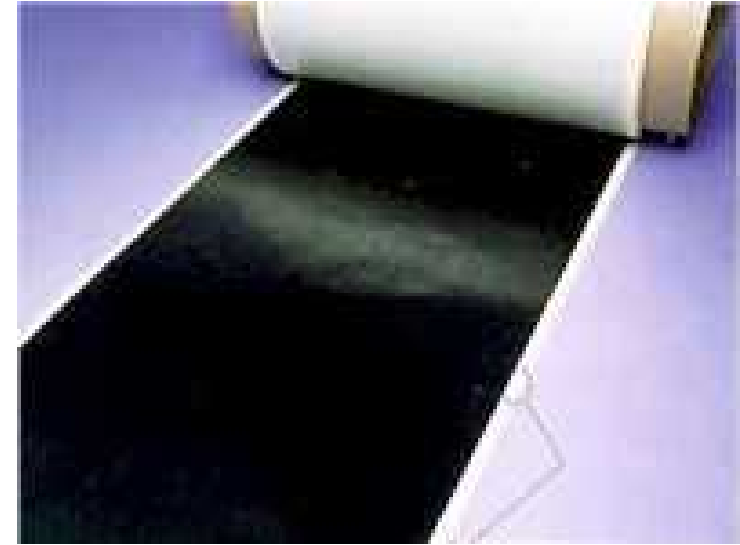

MBrace Carbon Unidirectional Fiber

\section{Technology}

Fiber reinforcement has been used for 25 years in aerospace and manufacturing applications where low weight, high tensile strength and noncorrosive structural properties are required. These field proven materials exhibit low creep and elongation, and compared to steel, they are thinner, lighter, and have 10 times the tensile strength capacity. The MBrace Composite Strengthening System, an externally bonded fiber reinforcement system for concrete and masonry structures, exhibits all of these properties.

\section{Strengthening Techniques Presentation}

- View HTML slide show

(Notes provided - audio or plug-in not required)

- View VIDEO slide show

(Narrated, video streaming, 28.8kbps, 43 minutes)

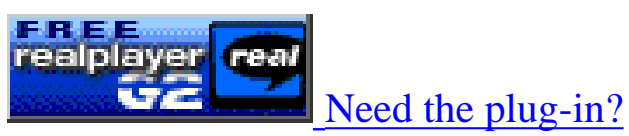

\section{Components}

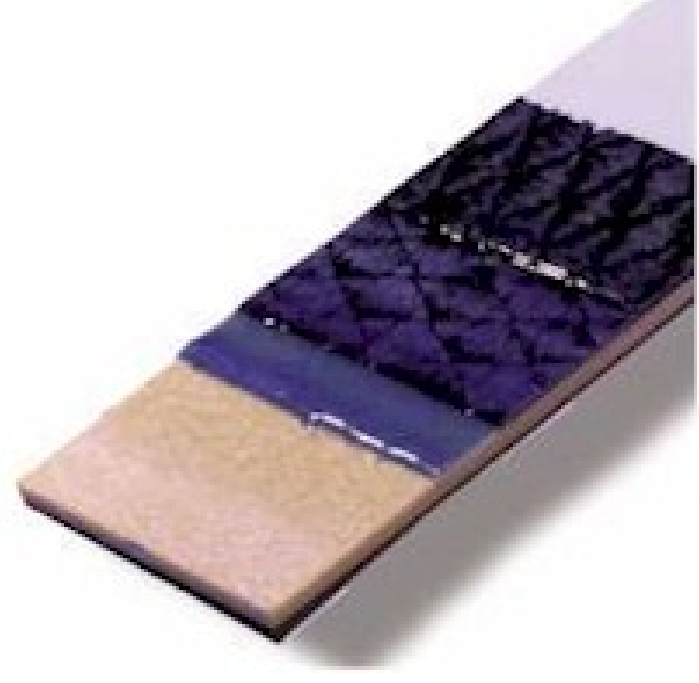

The MBrace system consists of advanced materials including:

- Surface primer

- Putty surface defect filler

- High solids epoxy saturant

- Carbon or e-glass fiber reinforcement

- Protective/aesthetic topcoat

The backbone of the MBrace system is the unidirectional continuous fiber sheet that is laminated with an epoxy matrix. Fiber/epoxy laminates, noted for their high strength-to- thickness ratio, establish structural integrity in a manner similar to bonding steel plates to structural elements. The result is tremendous flexibility for structural upgrades as well as significant savings relative to conventional strengthening methods.

Product Specification (Adobe Acrobat file 2 pages)

Need Acrobat Reader 3.0? \begin{tabular}{c} 
Get $\begin{array}{c}\text { Acrobat } \\
\text { Reader }\end{array}$ \\
\hline
\end{tabular}




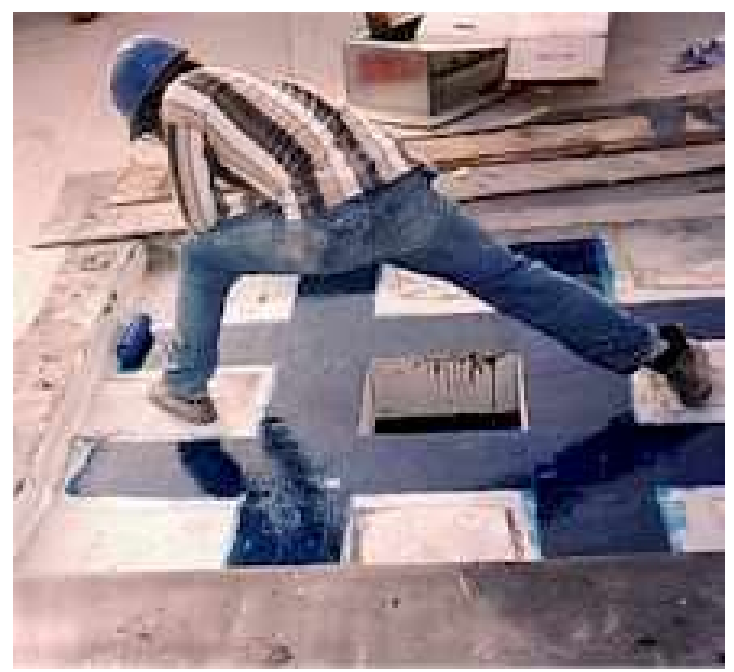

MBrace used to reinforce new slab opening
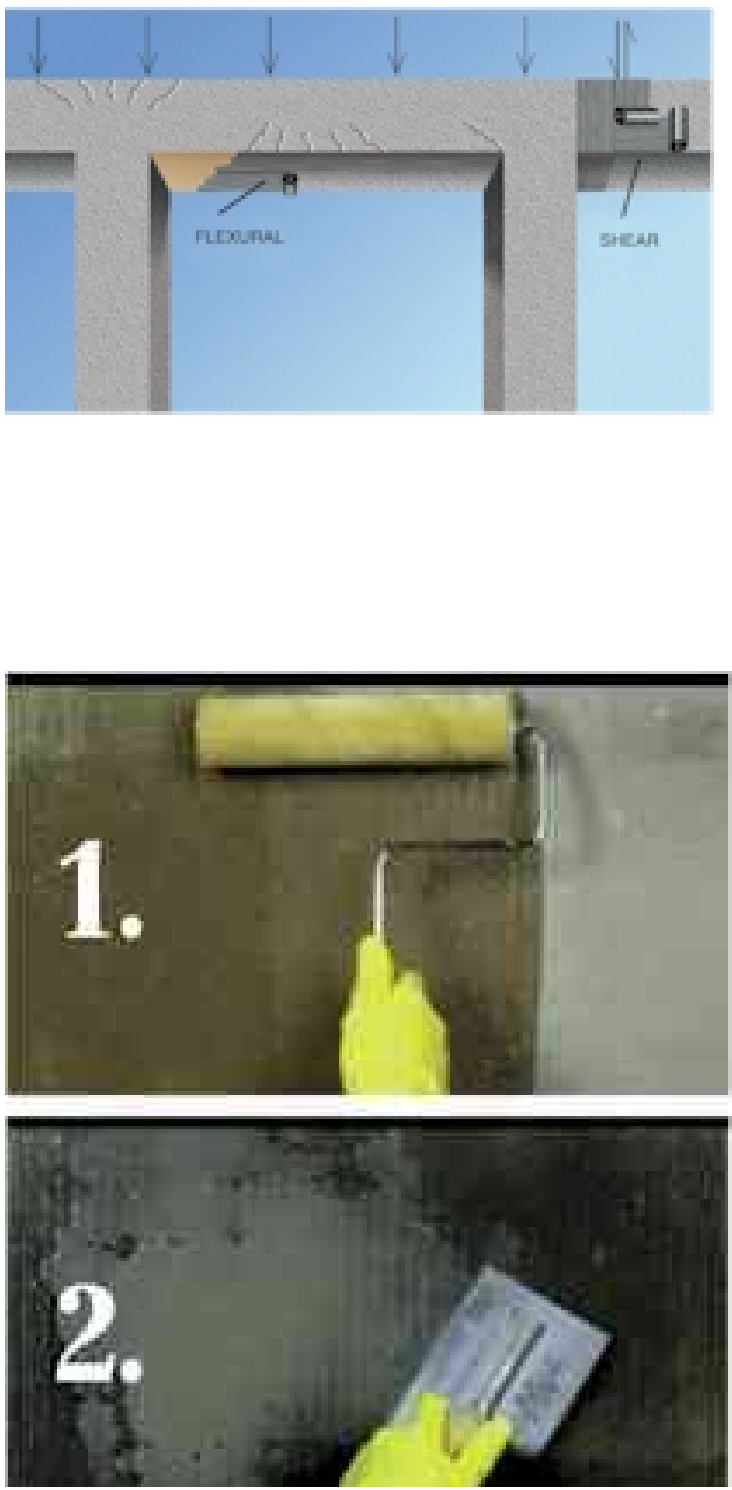

\section{Features \& Benefits}

Lightweight - Minimal additional dead load

Noncorrosive - High durability, low maintenance

Ease of Installation - Cost savings, minimal down time

Minimal Thickness - Minimal increase in member geometry, easily concealed

Flexible - Adapts to various shapes

10-Year History - Well-established technology

Single Source Support - Quality assurance through use of integrated components

\section{Applications}

MBrace technology provides solutions for strengthening beams, slabs, walls, columns, chimneys, silos, tunnels, tanks, and other structural elements that are subjected to deterioration, additional service loads or excessive deflection created by:

- Change in Use

- Construction or design defects

- Code changes

- Seismic retrofit

\section{Installation}

The MBrace system is installed exclusively within a national network of selected contractors. Each MBrace installation site requires the presence of trained contractors who are experienced in repair and strengthening strategies, product information, installation methods and QC testing.

The easy to use MBrace system components assure fast, user friendly installation. A complete system is installed in only six steps to properly prepared surfaces within appropriate working conditions.

\section{Roll MBrace Primer}

A low viscosity, high solids epoxy that can be applied using a roller. 


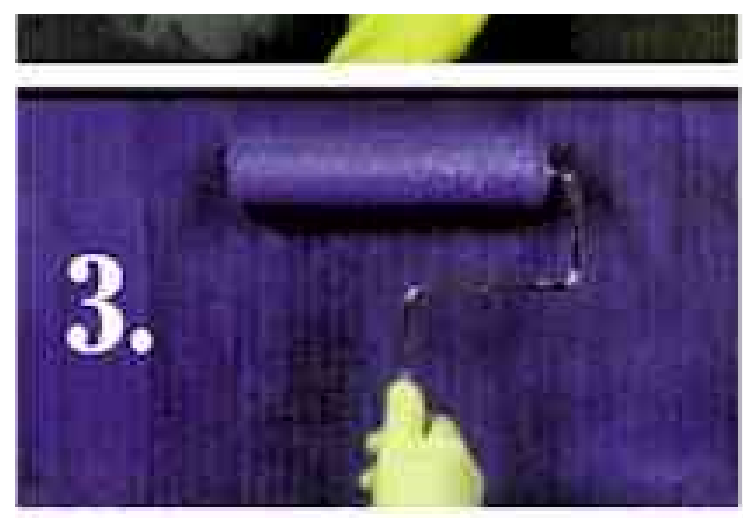

\section{Level Surfaces with MBrace Putty}

A high solids, nonsag paste epoxy material that is applied using a squeegee or trowel to level uneven surfaces.

\section{Apply First Coat of MBrace Saturant}

A high solids resin that can be applied using a roller to begin saturation of the fiber reinforcement sheet.

\section{Apply MBrace Fiber Reinforcement}

The backbone of the MBrace composite strengthening system, carbon fiber or e-glass sheet, is placed into the first layer of wet saturant and backing paper is removed.

\section{Apply Second Coat of MBrace Saturant}

The second coat of saturant is applied using a roller. For multiple plies, repeat steps $3,4, \& 5$.

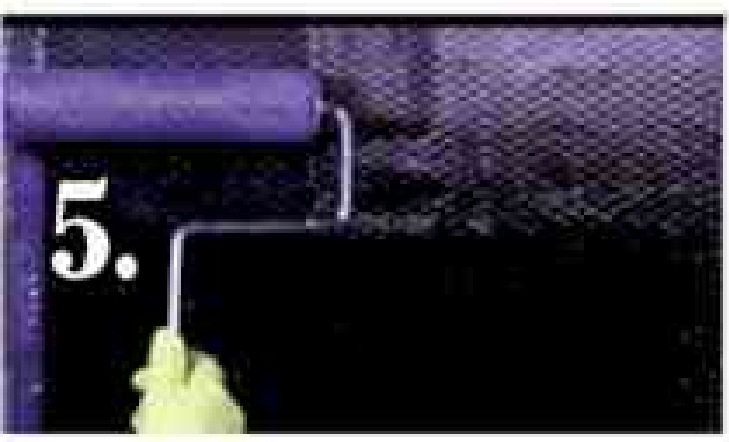

\section{Apply Optional MBrace Topcoat}

Where required, the high solids, high gloss, corrosion-resistant topcoat provides a protective/aesthetic outer layer.

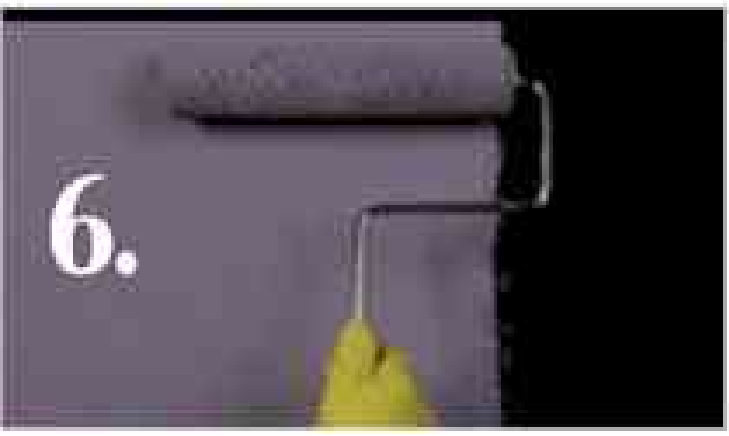

Need plug-in?

System Specification (Adobe Acrobat file, 12 pages)

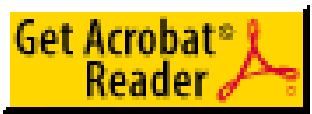


Need the plug-in? Installation Videos (real video format)

- MBrace Application on Pre-cast Double-Tee Stems Video streaming, 28.8kbps, 39 minutes

- MBrace Application of Beams to Increase Live Load Capacity

Video streaming, 28.8kbps, 40 minutes

If you reached this page from a search engine and would like to visit our complete site, click here. 
Related Bulletins:

Data Sheet

Design Guide
Master Builders Technologies

Restoration Products

SPECIFICATION BULLETIN

\section{MBrace $^{\mathrm{TM}}$ Composite Strengthening System}

with carbon fiber reinforcement for

CONCRETE SUBSTRATES

\section{NOTES TO THE SPECIFIER}

The specification information below is intended for use by architects, engineers or other specifiers in defining the criteria needed to specify carbon fiber reinforcement systems.

\section{CARBON FIBER REINFORCEMENT}

\section{PART 1 GENERAL}

\subsection{WORK INCLUDING}

A. Existing concrete shall be repaired and reinforced with dry, unidirectional carbon fiber fabric sheet.

B. The bid is deemed to include furnishings of materials, labor and equipment and all items necessary for repair and reinforcing of the concrete as specified on contract drawings and specifications, complete.

C. Drawings and the general provisions of the contract, including general conditions and general requirements are hereby made a part of this section.

D. Cooperate and coordinate with all other trades in executing the work described in the contract.

G. Inspect the structural members specified to be reinforced with Carbon Fiber Reinforced Plastic (CFRP) on the contract drawings to check the location and inspect cracks and existing conditions of beams.

H. Design and install CFRP laminates to reinforce [Beams, Slabs, Columns, Walls, or other].

\subsection{CODES AND REFERENCE STANDARDS}

A. Comply with provisions of the following codes, specifications and standards, except as otherwise indicated. Standard specifications of the applicable societies, Manufacturer's associations and agencies shall include the latest issues of the specifications. The Contractor shall have the following references at the project site at all times and shall be familiar with the reference contents.

1. State of Art Report on Fiber Reinforced Plastic Reinforcement for Structures (ACI 44OR-96).

2. Building Code Requirements for Structural Concrete (ACI 318-95) and (ACI 318R-95).

3. Pull-Out Test-Relates Pull-Out Resistance of Driven Pins to Concrete Strength (ACI 503R)

4. ICRI Surface Preparation Guidelines for Repair of Deteriorated Concrete Resulting from Reinforcing Steel Oxidation, selection of repair materials and placement of repair materials.

5. SACMA 4-88 Test method for tensile properties of oriental fiber resin composites.

6. Concrete Repair Guide (ACI 546R).

7. Guide to the Use of Waterproofing, Dampproofing, Protective, Decorative Barrier Systems for Concrete (ACI 515.R-85). 


\subsection{QUALITY CONTROL AND QUALITY ASSURANCE}

A. Manufacturer/Contractor Qualifications

1. Materials Manufacturer/Supplier Company must be specialized in the manufacturing of the products specified in this section.

2. Materials Manufacturer/Supplier Company must have been in business for a minimum of 5 years, with a program of training and technically supporting a nationally organized Contractor Training Program.

3. Contractor shall be a trained Contractor of the Manufacturer/Supplier of the specified product, who has completed a program of instruction in the use of the specified material.

B. Quality Control

The Contractor shall conduct a quality control program that includes, but is not limited to the following:

1. Inspection of all materials to assure conformity with contract requirements, and that all materials are new and undamaged.

2. Inspection of all surface preparation prior to CFRP laminate application.

3. Inspection of work in progress to assure work is being done in accordance with established procedures and established Manufacturer's instructions, specific Engineer Instructions, if given, or recommended practices listed in the references of Section 1.02.

4. Inspection of all work completed including sounding all repairs to check for debonding and correction of al defective work.

C. Quality Assurance

1. Attend pre-installation conference to be held with a representative of the Owner, Engineer, the Contractor's Superintendent and Foreman, Manufacturer's Field Representative and other trades involved to discuss the conduct of the work of this Section.

2. In-situ load testing of structural member prior to and after installation of CFRP sheet as required by these specifications. Quantity and location of member(s) to be tested shall be determined by Engineer of Record prior to proposal.

\subsection{SUBMITTALS}

A. Contractor's Qualifications

B. Manufacturer's product data indicating product standards, physical and chemical characteristics, technical specifications, limitations, installation instructions, maintenance instructions and general recommendations regarding each material.

C. Test results on the properties of the epoxy and the carbon fiber (CF) sheet / systems to be used on the project.

D. Provide a two year proven record of performance of strengthening projects with CFRP laminates, and five successful installations (in North America).

E. Provide field supervision specifically trained in the installation of CRFP laminates.

F. Samples of all materials to be used, each properly labeled as specified in Section 2.01.

G. Manufacturer's MSDS for all materials to be used.

$\mathrm{H}$. Certifications (in time to prevent delay in the work) by the Producers of the materials that all materials supplied comply with all the requirements and standards of the appropriate ASTM and other agencies.

I. Submit to the Owner's representative two copies of the design calculations and remedial design details prepared by the Contractor's professional Engineer using the CFRP laminates to be used on the job.

J. Submit stamped design drawings by a professional Engineer, including the necessary information listed above in a timely manner to obtain a building permit for the work. 
MBrace $^{\mathrm{TM}}$ Composite Strengthening System Specification

p 3 of 8

K. Adhesion testing process for 3.07-D1.

L. Load testing program (process, loads, shoring) as required.

\subsection{STRUCTURAL DESIGN}

A. Design the repair with CFRP laminates according to the design guides for the CFRP laminates and instructions supplied by the manufacturer.

B. Structural drawings of the existing structure included in the contract drawings.

\subsection{GENERAL PROCEDURES}

A. Work only in areas permitted by the Owner approved schedule.

B. Remove all tools, buckets and materials form work areas and store neatly at an approved location daily at the end of work.

C. Protect the building and its contents from all risks related to the work in this Section. Schedule and execute all work without exposing adjacent building areas to water, dust, debris or materials used by the Contractor. Protect adjacent areas from damage and stains with appropriate barriers and masking. Repair all damage as a result of the work to its condition at the start of work, or if such cannot be determined, to its original condition.

D. Protect the work from damage such as impact, marring of the surfaces and other damage.

F. Compliance with OSHA and all other safety laws and regulations is the exclusive responsibility of the Contractor, his Subcontractors, Suppliers, Consultants and Servants.

\subsection{TECHNICAL SUPPORT}

A. The Contractor shall provide the services of a trained field representative at the work site at all times to instruct the work crew in the CRFP application procedures.

1. The Manufacturer's Field Representatives must be fully qualified to perform the work.

2. The Contractor shall be completely responsible for the expense of the services of the required Manufacturer's Field Representative and the contract price shall include full compensation for all costs in connection therewith.

\section{PART 2 PRODUCTS}

\subsection{PRODUCT DELIVERY, STORAGE AND HANDLING}

A. Deliver materials clearly marked with legible and intact labels with Manufacturer's name and brand name, product identification and batch number.

B. The products shall be in original, unopened containers.

C. Store materials in areas where temperatures conform with Manufacturer's recommendations and instructions. 


\subsection{ACCEPTABLE MANUFACTURERS/SUPPLIERS}

A. The following vendors shall be used:

1. CFRP laminates: (Dry, unidirectional sheet only). MBrace Fiber Reinforcement Systems supplied by Master Builders, Inc. 23700 Chagrin Blvd., Cleveland, OH 44122 216-831-5500, 800-MBT-9990, Fax: 216-831-6910.

2. Epoxy resin adhesive: an approved epoxy system for application of MBrace Composite System. The system shall include:
a. Primer
b. Base Coat/Filler
c. Saturant
d. Topcoat

3. Substitutions: No substitutions allowed, except as requested by the Manufacturer/Supplier of the product and approved by the Engineer of Record.

\section{PART 3 EXECUTION}

\subsection{GENERAL PREPARATION FOR APPLICATION}

The contract drawings show locations of CFRP reinforcement.

\section{A. Ambient Temperature}

Conditions of CFRP process application must be examined carefully during the winter season and/or cold zones. DO NOT APPLY CFRP SHEET WHEN AMBIENT TEMPERATURES ARE LOWER THAN 40 degrees ${ }^{\circ} \mathrm{F}\left(5\right.$ degrees $\left.{ }^{\circ} \mathrm{C}\right)$. Auxiliary heat may be applied to raise surface and air temperature to a suitable range. Utilize "clean" heat source (electric, propane) so as not to contaminate bond surfaces by the carbonation of the substrate.

\section{B. Condensation}

Presence of moisture may inhibit adhesion or primer and/or resin. DO NOT APPLY CFRP WHEN RAINFALL OR CONDENSATION IS ANTICIPATED.

C. Concrete Surface Defects and Corners

UNEVEN CONCRETE SURFACE IRREGULARITIES (OFF SETS) MUST BE GROUND AND SMOOTHED TO LESS THAN 0.04 in. ( $1 \mathrm{~mm}$ ). WHEN CFRP SHEET IS TO RUN PERPENDICULARTO CORNERS, CONCRETE CORNERS MUST BE ROUNDED TO A RADIUS OF AT LEAST $0.5 \mathrm{in.} \mathrm{(15} \mathrm{mm).}$ INTERNAL CORNERS MUST BE SMOOTHED. NO DETAILING IS REQUIRED IF SHEET IS RUN PARALLEL TO CORNERS.

D. Handling of Primer and Resin

Refer to Manufacturer's specifications. DO NOT DILUTE PRIMER AND RESIN WITH ANY SOLVENT. After the resin has been mixed with hardener, the mixed resin batch must be used within its batch-life. The mixed batch resin must not be used after expiration of its batch-life because increased resin viscosity will prevent proper impregnation of CFRP Sheet.

E. Handling of CFRP Sheet

CFRP Sheet must not be handled roughly. CF Sheet must be stored either by being rolled to a radius greater than 12 in. $(300 \mathrm{~mm}$ ) or being dry stacked after cutting. When multiple lengths of CFRP Sheet are adhered to a concrete surface, a 4 in. (100 mm) OVERLAPPING LENGTH MUST BE APPLIED IN LONGITUDINAL (FIBER) DIRECTION. No overlapping is required in the lateral direction. 


\subsection{SURFACE PREPARATION}

A. All substrates must be clean, sound and free of surface moisture and frost. Remove dust, laitance, grease, curing compounds, waxes, impregnations, foreign particles and other bond inhibiting materials from the surface by blast cleaning or equivalent mechanical means. Any steel reinforcement should be cleaned and prepared thoroughly by abrasive cleaning, and the area patched prior to installation of CFRP laminates.

Any deteriorated concrete or corroded reinforcing steel must be repaired as per ICRI Specifications. Do not cover corroded reinforcing steel with CFRP.

B. Existing uneven surfaces must be filled with an appropriate repair mortar or must be ground flat. If required, the strength of the concrete or repaired area can be verified after preparation by random pull-off testing. Minimum tensile strength required is $200 \mathrm{psi}(1.4 \mathrm{MPa})$.

C. Prior to initiating surface preparation procedures, the Contractor shall first prepare a representative sample area. The sample area shall be prepared in accordance with the requirements of this Specification, and shall be used as a reference standard depicting a satisfactorily prepared surface.

D. (OPTIONAL) Where applicable, Contractor shall install a sample area ( $2 \mathrm{ft}^{2}$ or $\left.0.2 \mathrm{~m}^{2}\right)$ of CFRP for purposes of in-situ bond testing to verify bond. Cost for test to be additional.

E. Maintain control of concrete chips, dust and debris in each area of work. Clean up and remove such material at the completion of each day of blasting.

\subsection{APPLICATION STEPS}

A. The deteriorated surface layer of the base concrete (weathered layer, laitance, surface lubricants, broken mortar pieces, paint coatings, staining, etc.) must be removed and the surface ground using a disc sander or abrasive blasting.

Dusting from surface grinding must be removed using an air blower or other suitable means. If the dust has been removed by means of water washing, the surface must be thoroughly dried.

\section{B. Restoration of Concrete Cross Section}

Defects in the base concrete (such as broken pieces, voids, honeycomb, corrosion, etc.) must be chipped off and removed. If reinforcing bar has been exposed and corrosion exists, it must be repaired before the concrete restoration commences. The repair material shall be selected as per ICRI "Guide To Selecting Repair Material", and project requirements.

Epoxy resin or similar material must be injected into cracks greater than 0.010 in. $(0.25 \mathrm{~mm})$ wide.

If water leaks through cracks or concrete joints are significant, water protection and a water conveyance or run-off must be provided prior to concrete surface restoration.

\subsection{MIXING EPOXY RESIN}

A. Epoxy based material used in the composite system may develop higher viscosity and/or slow curing and insufficient curing at low ambient temperature. The ambient temperature of the epoxy components shall be between 50 and 100 degrees ${ }^{\circ} \mathrm{F}\left(10\right.$ to 38 degrees $\left.{ }^{\circ} \mathrm{C}\right)$ at the time of mixing. Presence of moisture may inhibit adhesion of the system to the concrete substrate. Provide necessary weather protection to protect surfaces from rain or cold.

B. Premix each component of the primer according to Manufacturer's recommendation. Use the appropriate mixing tools, at proper speed to achieve the proper mix. Take care to scrape the sides of the pail during mixing.

C. Components which have exceeded their shelf-life shall not be used.

D. Mix only that quantity of epoxy which can be used within its pot life. 


\subsection{APPLICATIONS}

A. No primer coat should be applied if the ambient temperature is lower that 40 degrees $F$ ( 5 degrees $C$ ), or if rainfall or condensation is anticipated.

1. Primer must be thoroughly mixed with hardener at the specified ratio in the mixing pot until it is uniformly mixed (about 2 minutes). Agitation shall be by means of electric hand mixer. Volume of primer prepared at one time must be such that it can be applied within its batch life. A mixed primer batch which has exceeded its batch life must not be used. (The batch life may vary subject to ambient temperature or volume of the mixed primer batch and care must be taken accordingly.)

2. Prime the concrete surface with the penetrating primer prior to application of any subsequent coatings using brush or roller. Alternatively, the primer may be spray applied with airless spray equipment, followed immediately by thorough back rolling to work the primer into the concrete surface. The primer shall be applied uniformly in sufficient quantity to fully penetrate the concrete and produce a nonporous film in the surface not to exceed two (2) dry mils (50 micrometers) in thickness after full penetration. Volume to be applied may vary depending on direction and roughness of the concrete surface.

3. Surface irregularities caused by primer coating must be ground and removed using disc sander, etc. If any minor protrusions on the concrete surface still remain, such surface defects may be corrected again using epoxy resin base coat/filler as needed.

4. Apply base coat/filler to primed surfaces with the penetrating primer to fill all substrate voids and irregularities. (See 3.01-C.)

\section{Adhesion of CFRP Sheet}

CF Sheet shall not be applied whenever ambient temperature is lower than 40 degrees $F$ ( 5 degrees $C$ ), or whenever rainfall or condensation is anticipated.

1. CF Sheet must be cut beforehand into prescribed sizes using scissors and/or cutter. The size of CF Sheet to be cut is preferably less than $10 \mathrm{ft}(3 \mathrm{~m})$ in length, but may be longer if access allows.

2. When the primer coat has been left unattended for more than one week after the application, the surface of the primer coat must be roughened using sandpaper. Do not solvent wipe.

3. Apply saturant coat to primed surface or CF sheet using a medium nap roller ( $3 / 8 \mathrm{in}$. or $9.5 \mathrm{~mm}$ ) to approximately 20 mil (500 micrometers) film thickness.

4. CF Sheet is placed fiber side down onto the concrete surface onto which the wet saturant coat has been applied. After smoothing down by hand, the backing paper is peeled away. The surface of adhered CF Sheet must be squeezed in the fiber longitudinal direction using a defoaming roller and rubber spatula in order to impregnate resin into CF Sheet and to defoam the resin coat.

For joining strips of CF Sheet in the fiber longitudinal direction, a 4 in. $(100 \mathrm{~mm})$ overlapping length is required. At the overlapping location, additional resin is applied to the outer surface of the CF Sheet layer to be overlapped. No lapping is required in the fiber lateral direction.

Minimize the elapsed time between mixing and application of the saturant to ensure the material is applied to the sheet at least 15 minutes prior to any thickening or gelling.

5. The CF Sheet shall have a minimum of 30 minutes between application of sheet into first coat of wet saturant on the concrete and the application of the second coat. This is to allow for epoxy impregnation.

When applying the first saturant coat directly to CF Sheet, allow for minimum of 30 minutes between resin application to sheet and second saturant coat. Less than 30 minutes can result in dry unsaturated areas of CFRP. Any listing or dislocation which may occur during this period must be corrected by pressing down the CFRP using a defoaming roller or spatula.

6. The secondary saturant coat of mixed resin must then be applied onto the surface of the CF Sheet. The surface onto which resin has been applied must be applied in fiber longitudinal direction, in order to impregnate and replenish resin into the CF Sheet using a roller in the same film thickness as detailed in 
Items 3 above.

7. In case more than one layer of CF Sheet must be laminated, the processes as detailed in Items 3 through 6 must be repeated.

8. In the case of outdoor application, the work must be protected from rain, sand, dust, etc. by using protective sheeting and other barriers. Curing of adhered CFRP must be for no less 24 hours.

\subsection{REPAIR OF DEFECTIVE WORK}

A. Repair of all the defective work after the minimum cure time for the CFRP laminates. Comply with material and procedural requirements defined in this specification. Repair all defects in a manner that will restore the system to the designed level of quality. Repair procedures for conditions that are not specifically addressed in this specification shall be approved by the Owner's representative. All repairs and touch up shall be made to the satisfaction of the Owner's representative.

\subsection{TESTING OF THE INSTALLED CFRP LAMINATES}

A. Test all the repaired areas to check for voids, bubbles and delaminations. Repair all voids, bubbles and delaminations by approved methods per manufacturer's direction.

B. Conduct direct pull-off test to verify the tensile bond between the CRFP and the existing concrete substrate. Inspect the failure surface of the core specimen. Failure at the bond line at tensile stress below $200 \mathrm{psi}(1.4 \mathrm{MPa})$ is unacceptable.

C. Perform a minimum of one pull-off test per $\mathrm{ft}^{2}\left(\mathrm{~m}^{2}\right)$ strengthened with the CFRP laminate system. The test is to be completed prior to the application of finishes on the CFRP laminates.

D. Repair the test areas of the composite system to the satisfaction of the Owner's representative.

\subsection{QUALITY CONTROL AND INSPECTION}

\section{A. In Process Control}

A certified inspector shall observe all aspects of onsite material preparation and application, including surface preparation, resin component mixing, application of primer, resin and CF Sheet, curing of composite, and the application of protective coatings.

B. Inspection for Void/Delaminations

After allowing at least 24 hours for initial resin cure to occur, perform a visual and acoustic tap test inspection of the layered surface. Large delamination shall be marked for repair. For small delaminations, which are typically less than $2 \mathrm{in.}^{2}\left(1300 \mathrm{~mm}^{2}\right)$ and which are not localized, do not require corrective action.

C. Adhesion Testing

Adhesion Test: The Contractor will conduct adhesion testing of the fully cured CFRP Sheet concrete assembly. (See 3.07.)

D. Load Testing

If required by Engineer, a representative area(s) determined by the Engineer of Record shall be in-situ load tested before and after application of CFRP Sheet to verify results.

E. Report

The inspector shall submit report to the Engineer of Record. 
MBrace $^{\mathrm{TM}}$ Composite Strengthening System Specification p 8 of 8

COMPONENTS PROVIDED BY: MASTER BUILDERS, INC.

Master Builders, Inc.

United States

23700 Chagrin Boulevard

Cleveland, Ohio 44122-5554

(800) MBT-9990

Fax (216) 831-6910

\section{Canada}

3637 Weston Road

Toronto, Ontario M9L 1W1

(800) 387-5862

Fax (416) 741-7925
Mexico

Blvd. M. Avila Camacho 80, 3er Piso 53390 Naucalpan, México

011-525-557-5544

Fax: 011-525-395-7903

\section{MASTER CONTRACTOR:}

Structural Preservation Systems, Inc.

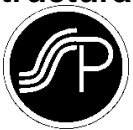

2116 Monumental Road

Baltimore, MD 21227-1633

800-899-1016

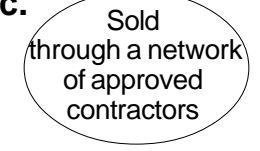

Visit us on the Web for the most current product information and news: www.masterbuilders.com 


\section{VITA}

John D. Barger

Graduate Research Assistant

BSCE_-West Virginia University, May 1997

MSCE—West Virginia University, May 2000 